MF

Publication 


\section{Democratic Republic of the Congo: First Review Under the Three-Year Arrangement Under the Extended Credit Facility and Financing Assurances Review-Staff Report; Staff Supplement; Press Release on the Executive Board Discussion; and Statement by the Executive Director for Congo, Democratic Republic of the. Democratic Republic of the Congo}

In the context of the first review under the three-year arrangement under the extended credit facility and financing assurances review, the following documents have been released and are included in this package:

- $\quad$ The staff report for the First Review Under the Three-Year Arrangement Under the Extended Credit Facility and Financing Assurances Review, prepared by a staff team of the IMF, following discussions that ended on June 30, 2010, with the officials of the Democratic Republic of the Congo on economic developments and policies. Based on information available at the time of these discussions, the staff report was completed on June 16, 2010. The views expressed in the staff report are those of the staff team and do not necessarily reflect the views of the Executive Board of the IMF.

- A Press Release summarizing the views of the Executive Board as expressed during its discussion of the staff report that completed the review.

- $\quad$ A statement by the Executive Director for the Democratic Republic of the Congo.

The documents listed below have been or will be separately released.

Enhanced Initiative for Heavily Indebted poor Countries-Completion Point Document and Multilateral Debt Relief Initiative Paper

Joint Staff Advisory Note of the Poverty Reduction Strategy Paper Progress Report

Letter of Intent sent to the IMF by the authorities of the Democratic Republic of the Congo*

Poverty Reduction Strategy Paper-Progress Report

*Also included in Staff Report

The policy of publication of staff reports and other documents allows for the deletion of market-sensitive information.

Copies of this report are available to the public from

International Monetary Fund $\bullet$ Publication Services

$70019^{\text {th }}$ Street, N.W. • Washington, D.C. 20431

Telephone: (202) 623-7430 • Telefax: (202) 623-7201

E-mail: publications@imf.org Internet: http://www.imf.org

\section{International Monetary Fund Washington, D.C.}




\title{
DEMOCRATIC REPUBLIC OF THE CONGO
}

\section{First Review Under the Three-Year Arrangement Under the Extended Credit Facility and Financing Assurances Review}

\author{
Prepared by the African Department
}

(In consultation with other departments)

Approved by Mark Plant and Christian Mumssen

June 15,2010

\section{EXECUTIVE SUMMARY}

Background. On December 11, 2009, the Executive Board approved a three-year arrangement under the Extended Credit Facility (ECF) for the Democratic Republic of the Congo (DRC) with total access of SDR 346.45 million (65 percent of quota) and an initial disbursement of SDR 49.49 million. A second disbursement in the same amount will become available upon completion of the first review.

Economic developments. Economic growth is estimated at $23 / 4$ percent in 2009 , in line with the program objective. It is expected to rebound to $5 \frac{1}{2}$ percent in 2010 on account of the recovery in the mining sector and higher investment. Inflation decelerated to 53 percent at end-2009 and further to 15 percent at end-April 2010. The external current account deficit declined by 6 percentage points of GDP in 2009 largely due to higher official transfers and lower income repatriation associated with weak mining activity. International reserves rose from the historic low of one week of nonaid imports in early 2009 to 7 weeks by year-end, boosted largely by the general and special SDR allocations and Fund financial assistance.

Program implementation. Good revenue performance helped contain the 2009 fiscal deficit below the program target and reduced the government's reliance on borrowing from the banking system. The good fiscal performance continued through March 2010. The central bank tightened monetary policy in second half of 2009, but began to ease its stance in March 2010 in response to falling inflation. Program implementation was satisfactory and all quantitative performance criteria at end-December 2009 were observed. The authorities also implemented almost all of the structural benchmarks, albeit with delays.

Policy recommendations. Fiscal policy should focus on reducing government recourse to central bank financing while creating the fiscal space for priority programs by bolstering revenue and strengthening expenditure management. Further easing of monetary policy should wait until inflationary pressures are fully contained. Structural reforms need to focus on strengthening public financial management (PFM) and addressing financial sector weaknesses. Staff supports the authorities' request for completing the review.

Risks. Escalation of conflict in the eastern provinces, negative terms of trade shock, and election-related expenditure pressures could undermine program implementation. Moreover, risk of debt distress is expected to remain high even after debt relief. Donor support, including for peacemaking efforts and elections, could help mitigate risks to program implementation.

Discussions. They were held in Kinshasa during March 2-20, 2010, and in Washington, D.C. during April 22-27, 2010. The mission comprised Messrs. Ames (Head), Farah, and Ms. Bovha-Padilla (all AFR), and Messrs. Callegari (FAD) and Hostland (SPR). Mr. Jahjah, resident representative, also participated in the discussions. The team worked closely with the World Bank resident office in Kinshasa. 
Acronyms

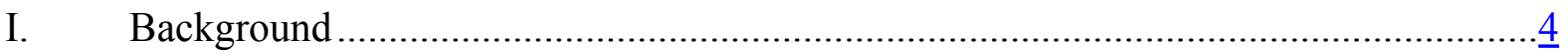

II. Recent Developments and Performance under the Program ................................... 4

III. Policy Discussions ................................................................................. 10

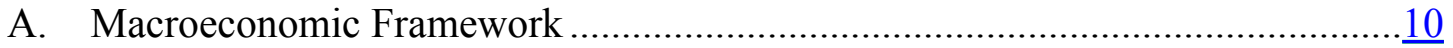

B. Macroeconomic Policies ............................................................................ $\frac{10}{13}$

C. Structural Reforms ..........................................................................

IV. Capacity to Repay the Fund and Risks ..........................................................13

V. Staff Appraisal .....................................................................................

Figures

1. Recent Economic Developments ................................................................. 6

Tables

1. Selected Economic and Financial Indicators, 2007-15 …....................................16

2a. Central Government Financial Operations, 2007-12 …......................................17

2b. Central Government Financial Operations, 2007-12 …........................................ 18

3a. Monetary Survey, 2007-10.........................................................................19

3b. Accounts of the Central Bank of the Congo, 2007-10 ….....................................20

4. Balance of Payments Summary, 2007-15 .............................................................21

5. Tentative Schedule of Disbursements and Reviews under the ECF Arrangement, $2010-12$....................................................................... 22

6. Indicators of Capacity to Repay the Fund, 2010-22 ..........................................23

Appendix I

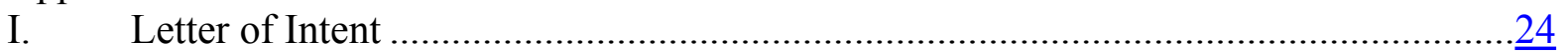

Appendix I Attachments

I. Memorandum of Economic and Financial Policies ...............................................26

Table 1.1 Quarterly Quantitative Performance Criteria and Indicative Targets, 2009-10 ….......................................................................

Table I.2a Structural Benchmarks, July 2009-June 2010 …..............................33

Table I.2b Structural Conditionality and Macroeconomic Relevance, July-December 2010 ..........................................................

II. Technical Memorandum of Understanding ….................................................. 43 


\section{ACRONYMS}

$\begin{array}{ll}\text { BCC } & \text { Central Bank of the Congo } \\ \text { BTR } & \text { Central bank bills } \\ \text { CGF } & \text { Congolese franc } \\ \text { DRC } & \text { Democratic Republic of the Congo } \\ \text { ECF } & \text { Extended Credit Facility } \\ \text { EITI } & \text { Extractive Industries Transparency Initiative } \\ \text { HIPC } & \text { Heavily Indebted Poor Countries } \\ \text { JSAN } & \text { Joint Staff Advisory Note } \\ \text { MEFP } & \text { Memorandum of Economic and Financial Policies } \\ \text { MCM } & \text { Monetary and Capital Markets Department } \\ \text { MDRI } & \text { Multilateral Debt Relief Initiative } \\ \text { MEFP } & \text { Memorandum of Economic and Financial Policies } \\ \text { MONUC } & \text { United Nations Mission in the Democratic Republic of the Congo } \\ \text { NCG } & \text { Net credit to government } \\ \text { NDA } & \text { Net domestic assets } \\ \text { NFA } & \text { Net foreign assets } \\ \text { OHADA } & \text { Organization for the Harmonization of Business Law in Africa } \\ \text { PFM } & \text { Public financial management } \\ \text { PRGS } & \text { Poverty Reduction and Growth Strategy } \\ \text { PV } & \text { Present value } \\ \text { REER } & \text { Real effective exchange rate } \\ \text { SCCA } & \text { Sino-Congolese Cooperation Agreement } \\ \text { TMU } & \text { Technical Memorandum of Understanding } \\ \text { VAT } & \text { Value added tax }\end{array}$




\section{BACKGROUND}

1. The DRC authorities' economic program aims to enhance macroeconomic stability and advance reforms to bolster economic management and improve the supply response of the economy. In support of the program, on December 11, 2009, the Executive Board approved a three-year arrangement under the ECF (July 2009-June 2012) with total access of SDR 346.45 million (65 percent of quota) and an initial disbursement of SDR 49.49 million.

\section{Progress in implementing the authorities' Poverty Reduction and Growth Strategy}

(PRGS) has been affected negatively by the continuation of conflict. The authorities have prepared a report on progress in implementing their first generation PRGS ${ }^{1}$ and are in the process of preparing their second-generation strategy. The IMF and World Bank staffs have prepared a Joint Staff Assessment Note (JSAN) on the authorities' report. ${ }^{2}$

3. The security and political situation remains calm but uncertain. The March 2009 peace agreement between the government and rebels in the eastern provinces is holding, but the reintegration and disarmament program is moving slowly. In early 2010, ethnic based rebels attacked and briefly held the capital of a northwestern province before government forces supported by the United Nations (MONUC) regained control. A cabinet reshuffle in March 2010 preserved the ruling coalition government, but new ministers in charge of budget and finance were appointed.

\section{Recent Developments and Performance Under the Program}

4. The DRC economy was adversely affected by the global financial crisis, which triggered steep declines in commodity export prices and a slowdown in mining activity and foreign direct investment. The domestic commercial bank system was insulated from contagion from the international banking system due to the low level of foreign bank participation. It was nevertheless affected by the impact of the crisis on the real economy. There are signs, however, that economic conditions are improving.

- Economic activity: Growth is estimated at 2.8 percent in 2009 (down from 6.2 percent in 2008) on account of a deterioration in the country's terms of trade during the first half of the year and a reduction in domestic demand (Figure 1, and Table 1). Although mining sector performance began to rebound during the second half of the year due to the firming up of world copper prices, the services sector expanded more slowly than projected. Preliminary indicators for the first quarter of 2010 point to continued buoyant copper production in the context of higher world commodity prices. Annualized inflation decelerated to about 53 percent at end-2009—-some 5 percentage points higher than

\footnotetext{
${ }^{1}$ www.imf.org.

${ }^{2}$ www.imf.org.
} 
programmed - and further to 15 percent in April 2010. The Congolese franc (CGF) appreciated modestly against the U.S. dollar during the first quarter of 2010, after depreciating by 49 percent in 2009 . The real effective exchange rate remained broadly unchanged, with higher domestic inflation offsetting the depreciation of the nominal effective exchange rate.

- External sector. In 2009, despite the steep fall in the terms of trade, the current account deficit narrowed by some 6 percentage points of GDP because of declines in income repatriation - mainly from mining - and higher official transfers. Financial account inflows declined largely because of the reduction in foreign direct investment. Gross international reserves rose to the equivalent of 7 weeks of nonaid imports by end-2009, up from the historic low of one-week coverage a year earlier. This was largely on account of the special and general SDR allocations, disbursements under the ECF arrangement and the rapid access component of the Exogenous Shocks Facility, and the first tranche of the Sino-Congolese Cooperation Agreement (SCCA) signing bonus.

- The financial sector remains fragile. Commercial bank profits and return on equity fell sharply in 2009, a number of banks face difficulties in meeting prudential regulation requirements, and there was an increase in nonperforming loans. The Central Bank of the Congo (BCC) has increased its on-site inspection of commercial banks and is developing restructuring plans for the weakest ones. It also provided liquidity support for a financially distressed large commercial bank. Dollarization remains high and dollardenominated deposits rose further in 2009, which constrains the BCC's role as the lender of last resort in the event of a banking crisis given its low level of international reserves. 
Figure 1. Democratic Republic of the Congo: Recent Economic Developments

The global recession curbed GDP growth...

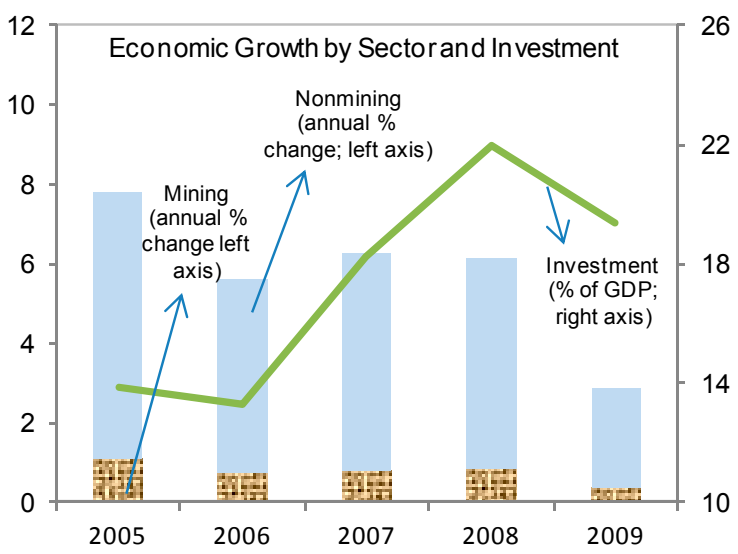

But, a recovery of copper prices helped strengthen mining activity since the second half of 2009.

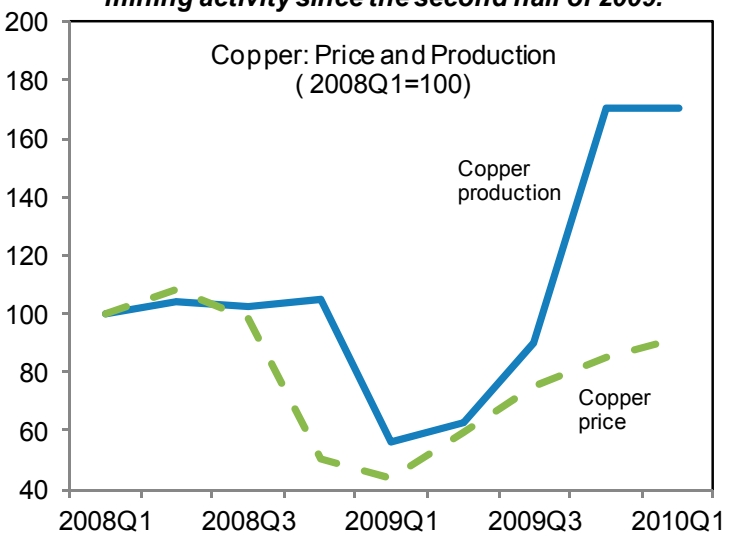

... while banks'returns declined ...

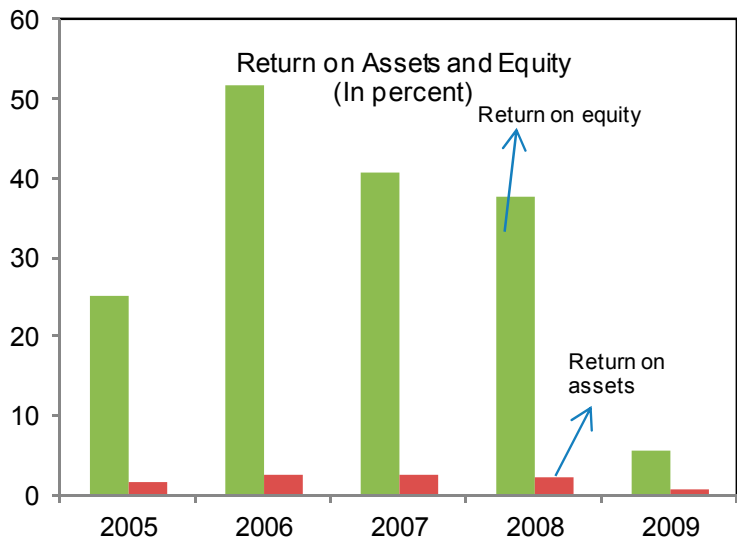

... and slowed the expansion of private sector credit.

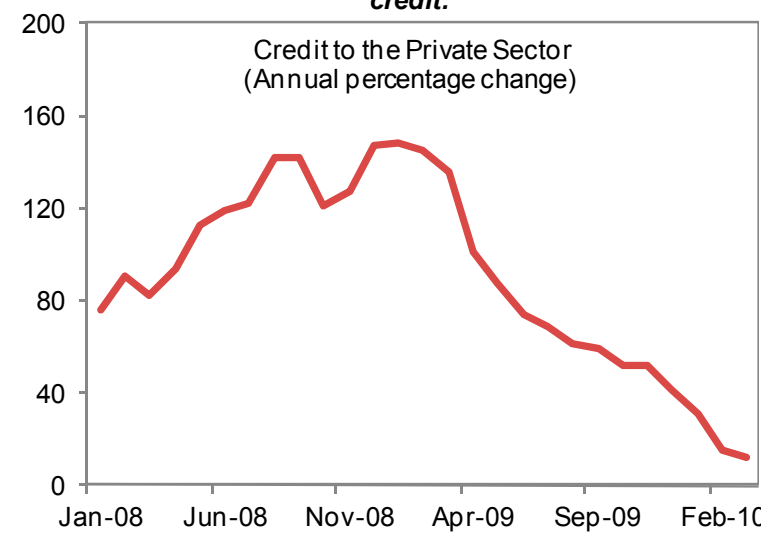

Nonperforming loans in the banking sector rose in 2009...

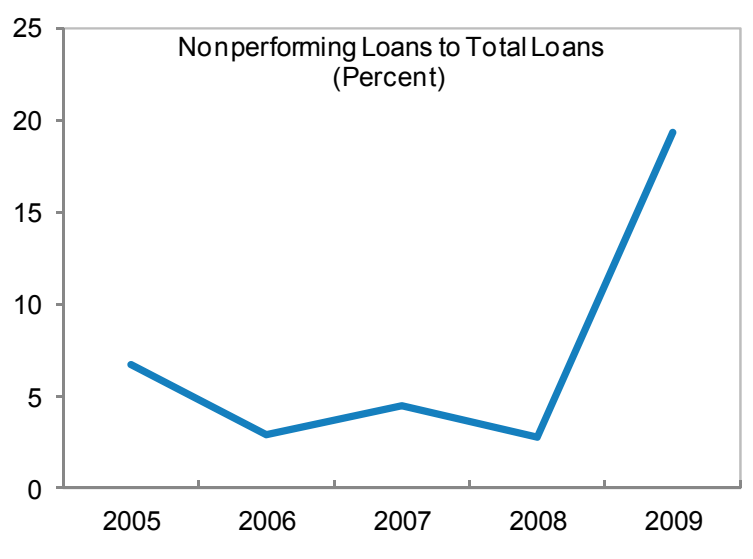

... and dollarization increased.

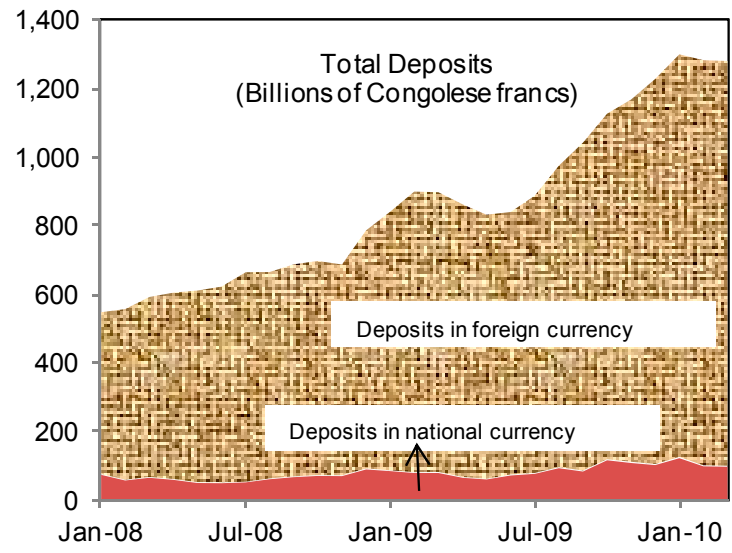

Sources: Congolese authorities and IMF staff estimates. 
Figure 1. Democratic Republic of the Congo: Recent Economic Developments Buoyant revenue during the second half of 2009

(Concluded) and early 2010 ...
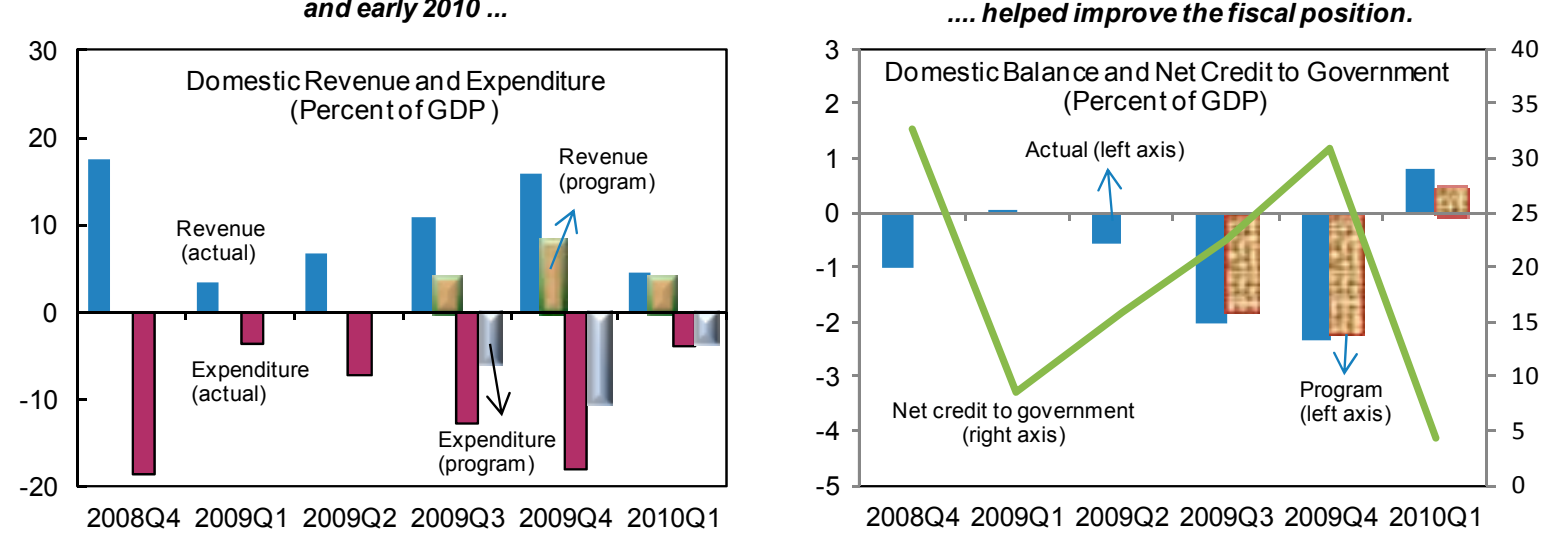

Monetary policy was eased in early 2010, after tightening in the second half of $2009, \ldots$
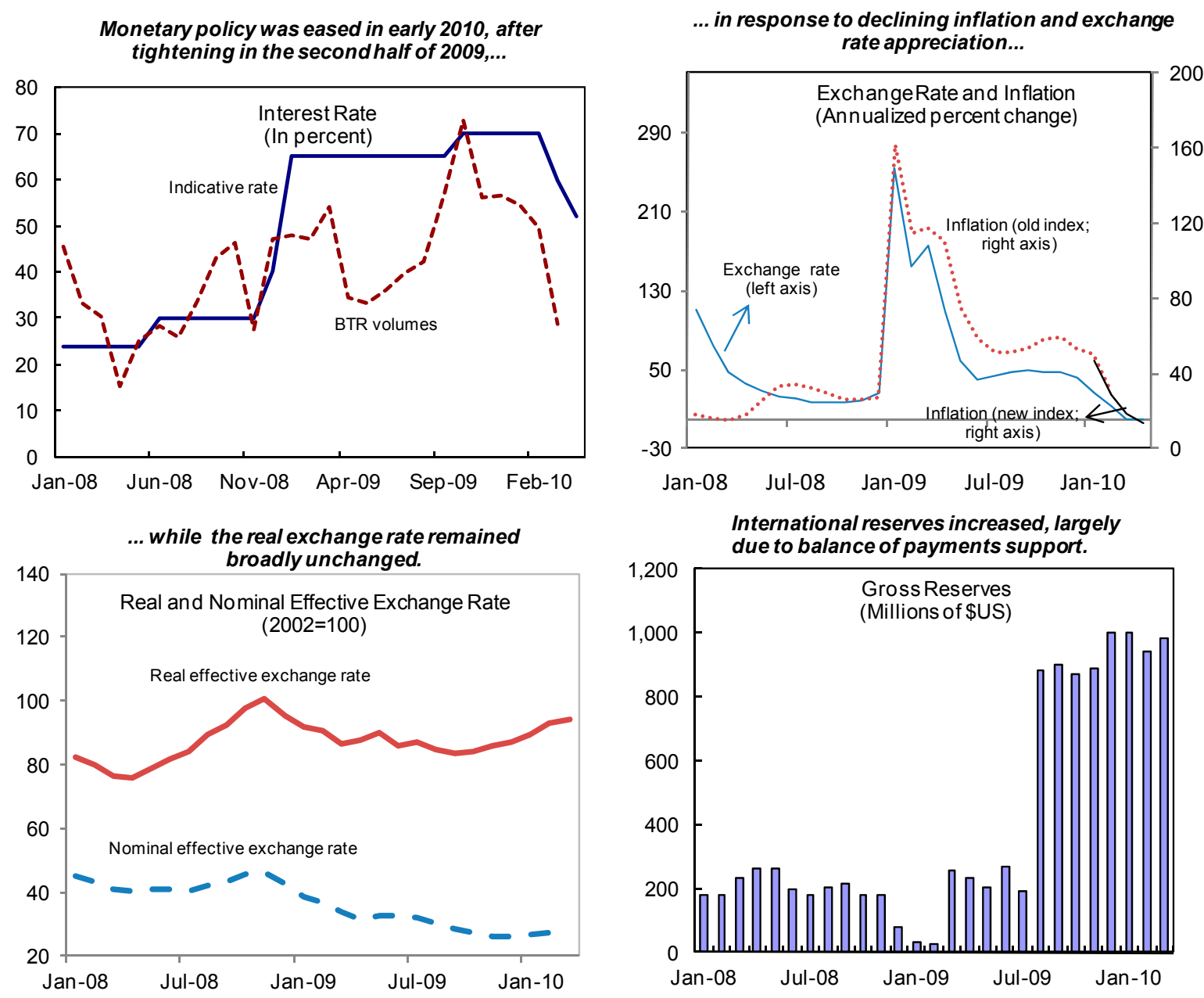

Sources: Congolese authorities and IMF staff estimates. 
5. Macroeconomic policies were geared to mitigate the impact of the global financial crisis on the domestic economy.

- The authorities allowed the $\mathbf{2 0 0 9}$ domestic fiscal deficit to widen relative to 2008 while curtailing recourse to central bank financing (Table 2). The deficit increased by 2.2 percentage points of GDP ( 0.6 percentage points lower than programmed) to 2.5 percent while the share of investment in total spending increased. The loss of revenue from the slowdown of economic activity amounted to 2.8 percent of GDP-

0.8 percentage points less than projected — thanks to higher receipts from the mining sector. ${ }^{3}$ Although domestically financed spending declined by some 0.4 percentage points of GDP to 18.3 percent, the share allocated to investment in infrastructure increased. ${ }^{4}$ This, along with increased (yet lower than projected) external budget support, reduced net credit to the government from the banking system (NCG). During the first quarter of 2010 , revenues remained strong ( 0.7 percentage points of GDP above projection) while there were modest spending overruns ( 0.4 percentage points of GDP) mainly on national security, preparation for the $50^{\text {th }}$ independence anniversary, and transfers to cover central bank operating losses arising from the cost of monetary policy operations. ${ }^{5}$ Thus, NCG declined further.

- The BCC tightened monetary policy in late 2009 , but has recently begun to ease its stance. It raised its policy interest rate to 70 percent in October 2009- the third upward adjustment during the year-in response to pressures on the exchange rate and inflation. However, because of weak liquidity forecasting, base money grew faster -5 percentage points - than targeted at end-year (Table 3). During the first quarter of 2010, the continued good fiscal performance and improvement in the BCC's liquidity forecasting capacity (including through closer collaboration with the Treasury) enhanced monetary control. In response to the decline in inflation, the $\mathrm{BCC}$ cut its policy interest rate to 42 percent in three steps by May 2010.

\section{Paris Club creditors provided exceptional treatment to the rescheduling of the}

DRC's external debt. The February 2010 rescheduling agreement reduced the amount of debt service (including arrears) due to Paris Club creditors by 97 percent over the period covered by the ECF arrangement. The authorities have been in contact with their other external creditors, informed them about the terms of the rescheduling agreement reached with the Paris Club in February 2010, and have engaged into negotiations seeking rescheduling agreements on

\footnotetext{
${ }^{3}$ To facilitate comparison, the ratios of fiscal indicators-to-GDP of the original program projections (IMF Country Report No. 10/88) were recalculated using the latest GDP estimates.

${ }^{4}$ Domestically financed spending also includes repayment of arrears and the BCC deficit.

${ }^{5}$ The operating deficit of the BCC during the first quarter of 2010 was at CGF 30 billion, almost the entire amount budgeted for the year.
} 
comparable terms. ${ }^{6}$ Currently the DRC is in the process of negotiating a buy-back operation supported by IDA's Debt Reduction Facility to extinguish the remaining eligible commercial claims under the Heavily Indebted Poor Countries (HIPC) Initiative as of end-December 2009.

\section{An update safeguards assessment of the BCC was completed in April 2010. It found}

that the majority of 2008 recommendations have been implemented, including establishing procedures for external audit of the biannual program-data. However, significant safeguards risks remain. The BCC continues to lack autonomy from the government and is in urgent need of recapitalization. Furthermore, the absence of a benchmark financial reporting framework continues to impair transparency. The BCC broadly agreed with the priority recommendations of the assessment and has made some progress towards their implementation. Some risks remain for the accurate reporting of NCG; however, as the balance of the Treasury General Account is not regularly reconciled between the BCC and the Ministry of Finance. The auditor's report did not identify any breach of the audited performance criteria during the period from December 31, 2008, to December 31, 2009. The status of safeguards recommendations will continue to be monitored under the program.

\section{Program performance was satisfactory. All quantitative performance criteria and} indicative targets at end-December 2009 were observed, except for the indicative targets on base money and the accumulation of wage arrears (Memorandum of Economic and Financial Policies (MEFP), Table I.1). ${ }^{7}$ Three of the quantitative indicative targets at end-March 2010 were not observed (that is, net foreign assets (NFA), net domestic assets (NDA), and base money). This reflected a larger-than-envisaged share of foreign currency denominated government spending (mainly security related), and higher commercial bank recourse to the BCC's short term lending facility to meet liquidity demand by the private sector to settle quarterly tax obligations. The $\mathrm{BCC}$ has since increased purchases of foreign exchange from the market and commercial banks have reversed their borrowing from the central bank (MEFP, -4). Almost all of the structural benchmarks were implemented, albeit with delays (MEFP, $₫ 5$ and Table I.2.). ${ }^{8}$ The benchmark on the production of fiscal accounts that include foreign financed spending was not met because of inadequate reporting from nongovernmental organizations that manage foreign financed projects. There was good performance in other structural areas such as the adoption of an action plan to strengthen PFM and promulgation of the protocol of the Organization for the

\footnotetext{
${ }^{6}$ Arrears to BDGEL (Banque de Development des Etats des Grand Lacs) (US\$80.2 million at end-December 2009) are programmed to be cleared in light of the ongoing discussions between the DRC authorities and BDGEL representatives.

${ }^{7}$ The accumulation of wage arrears vis-à-vis diplomats based overseas during the last quarter of the year amounted to CGF 2 billion or 0.4 percent of the total wage bill. This was because wage payments to diplomats are made outside the automated system in place for all other civil servants.

${ }^{8}$ Staff considered that the benchmark on restructuring one large bank was met based on the central bank's agreement to appoint a Provisional Administrator. The restructuring itself will require more time and is being carried out with technical assistance from the IMF's Monetary and Capital Markets (MCM) Department.
} 
Harmonization of African Business Law (OHADA), which is intended to help reduce the cost of doing business. The authorities have also begun preparation to adhere to the Extractive Industries Transparency Initiative (EITI).

\section{Policy Discussions}

9. Discussions focused on the 2010 macroeconomic framework, macroeconomic policies, and structural reforms in tax and customs administration, PFM, financial sector, and improving the business climate.

\section{A. Macroeconomic Framework}

10. The economic outlook is improving. Real GDP growth is expected to reach 5.4 percent on the back of a strong recovery in the mining sector and increased investment in mining and public infrastructure. The curtailment of government recourse to central bank financing should help the BCC better control monetary aggregates and contain inflation to 15 percent by end2010. Despite the projected increase in mining exports, the current account deficit is expected to widen largely due to higher imports tied to infrastructure and mining projects. However, increased foreign direct investment and financing under the SCCA should help improve the capital and financial accounts. Nevertheless, there is a financing gap of about US\$198 million million after taking into account the expected disbursements under the ECF arrangement of US\$146 million (SDR 98.99 million) and the need to maintain reserves at the equivalent of 7.5 weeks of nonaid imports (Table 4). Financial support from multilateral and bilateral development partners is expected cover the gap (see $\uparrow 15$ below).

\section{B. Macroeconomic Policies}

\section{Fiscal policy}

\section{The authorities are committed to a fiscal policy that further curtails recourse to} central bank financing. The 2010 budget approved by Parliament contained overly optimistic revenue assumptions (some 2 percentage points of GDP greater than under the ECF-supported program) and commensurately higher spending. However, the authorities reconfirmed their commitment to implement a more prudent fiscal policy consistent with the program's objectives. The domestic fiscal deficit is targeted to narrow to 1 percent of GDP, and the overall fiscal deficit will be limited to 5.1 percent of GDP. This would allow NCG to decline by 1.5 percentage points of GDP compared with the zero change envisaged under the original program projections. The larger deposit build up reflects: (i) a better revenue outlook and the authorities' decision to save a portion of the windfall, and (ii) the full saving of the second tranche of the signing bonus under SCCA (1 percentage point of GDP). The revised program envisages revenue-enhancing measures and reprioritization of spending in order to create the fiscal space for priority programs not previously envisaged under the program. In particular: 
- Revenue is now projected at 18.1 percent of GDP in 2010, 1.4 percentage points of GDP higher than originally programmed. The revised projection takes into account: (i) higherthan-programmed revenue performance during the first quarter of the year $(0.7$ percent of GDP); (ii) a gradual alignment of the price used for the calculation of taxes on petroleum products (prix fiscale) with world prices ( 0.3 percent of GDP);

(iii) enforcement of the payment of income taxes by political institutions $(0.2$ percent of GDP); and (iv) elimination of ad hoc exemptions on income taxes and import tariffs (0.2 percent of GDP). ${ }^{9}$ The program also includes structural reforms that should help boost revenue over the medium term (see $\uparrow 16$ below).

- Domestically financed spending is now capped at 19.1 percent of GDP (about

1.1 percentage points higher than the original program target). In order to create the fiscal space for additional spending on national security and the national elections, the authorities plan to cut domestically financed capital expenditure (by about 0.6 percentage points of GDP) ${ }^{10}$ The revised fiscal program also accommodates: (i) a larger BCC deficit (0.3 percent of GDP) that takes account of the higher-than-budgeted costs of monetary policy; (ii) payment of government arrears ( 0.2 percent of GDP) to a commercial bank that is undergoing restructuring; and (iii) clearance of arrears vis-à-vis the provinces and tax-collection agencies ( 0.4 percent of GDP). To mitigate risks to the fiscal program in the event of a revenue and/or external budget support shortfall, the authorities identified contingent spending reduction equivalent to 0.5 percent of GDP in capital expenditure and domestic arrears payments.

- Foreign financed investment and exceptional spending are projected at about 13.3 percent of GDP, 7 percentage points higher than 2009. A large portion of the increase is explained by the infrastructure projects under the SCCA and outlays associated with the national and provincial elections slated for 2011.

\section{Staff stressed that effective implementation of the recently adopted tax measures and strict expenditure control are essential for achieving the program's fiscal objectives.}

The authorities concurred and noted that the recent introduction of new expenditure management procedures that also apply to urgent spending will help strengthen expenditure control. Staff raised concerns over the proposed cuts in government investment and their implications for maintenance of public infrastructure. The authorities maintained that the spending cuts would have limited adverse effects in light of the significant investment financed under the SCCA (5.6 percent of GDP). Given the country's susceptibility to exogenous shocks, staff fully supports the authorities' decision to save a portion of the projected higher domestic revenue

\footnotetext{
${ }^{9}$ These relate to tariffs on petroleum imports for mining companies and income taxes for commercial banks.

${ }^{10}$ The original program included CGF 11 billion (0.1 percent of GDP) for elections and the $50^{\text {th }}$ independence anniversary celebrations.
} 
(relative to the original program) and the full amount of the second tranche of the SCCA signing bonus.

\section{Prudent fiscal policy, supported by concessional financing, will also be critical for} debt sustainability. Given that that the HIPC debt sustainability analysis indicates that the DRC will continue to face high risk of debt distress even after receiving substantial debt relief under the HIPC Initiative and the Multilateral Debt Relief Initiative (MDRI), ${ }^{11}$ the authorities committed to rely on grants and highly concessional loans (i.e., with a grant element of at least with 35 percent) to meet their external financing needs (MEFP, $₫ 10$ ).

\section{Monetary and exchange rate policies}

\section{Monetary policy will continue to focus on achieving the program's year-on-year} inflation objective of 15 percent by end 2010. Staff and the authorities agree that keeping the central bank policy interest rate significantly positive in real terms is critical to avoiding the reemergence of pressures on the exchange rate and inflation. Although staff supported the easing of monetary policy given the deceleration of inflation during the first quarter of 2010, it noted that the pace and magnitude of easing (a 32 percent reduction in the policy interest rate in three steps since March) could have been more gradual and smaller to minimize the risks to the program's inflation objective. Staff believes that the policy rate should remain unchanged going forward until inflation has been fully contained. Reserve requirements should also remain "as is" for the time being. ${ }^{12}$ The implementation of monetary policy will be facilitated by the enhanced collaboration between the central bank and the Treasury regarding liquidity forecasting. The authorities and staff agreed that the flexible exchange rate regime continues to be appropriate given the country's relatively low level of international reserves and its susceptibility to exogenous shocks. BCC intervention in the foreign exchange market will therefore continue to be limited to smoothing short-term exchange rate volatility.

\section{Program financing}

15. The program is fully financed. The financing gap is estimated at US\$198 million over the period July-December 2010. Staff expects this gap to be covered by budget support from the World Bank (US\$100 million), the European Commission (US\$67 million), and bilateral donors (US\$31 million). The program also assumes that completion point is reached by end-2010 and all creditors provide debt relief in accordance with the HIPC Initiative and MDRI. Official grants projected over the program period (Table 2) include debt relief under the HIPC Initiative and MDRI and assume that donors will respond favorably to the DRC reaching the completion point, which is essential if the country's debt is to be sustainable over the medium term.

\footnotetext{
${ }^{11}$ See www.imf.org.

12 The BCC raised the reserve requirement from 5 percent to 7 percent in October 2009. The monetary authorities remain concerned over the impact of such high reserve requirements on financial intermediation.
} 


\section{Structural Reforms}

16. Structural reforms for the remainder of 2010 will continue to focus on revenue mobilization, PFM, financial sector solvency, and private sector development (MEFP, $\mathbf{q} \uparrow 12-15$, and Table I.2b).

Revenue administration will be strengthened through the adoption by parliament of a value added tax (VAT) law, establishment of new tax centers in key provinces for medium-sized enterprises, and expansion of one-stop windows at border posts (MEFP, $₫ 13$ ).

PFM will be improved through the adoption of a new finance law that will streamline budget planning, execution, and monitoring. The authorities are cognizant of the risks associated with fiscal devolution given the weak PFM capacities at the provincial level. In this context, the new finance law will also set the stage for a phased devolution of social expenditures from the central government to the provinces, which takes into account the PFM capacities at the provincial level (MEFP, $\llbracket 13$ ).

Financial sector reform will focus on rebuilding central bank independence and addressing weaknesses in the commercial banking sector (MEFP, $\uparrow 14)$. Key reform measures to help improve the financial independence of the central bank include the adoption by parliament of a recapitalization plan, development of a privatization strategy for the Mint and the BCC hospital, and reform of the BCC's pension system. Regarding commercial banks, the BCC will complete on-site banking supervision inspections of the commercial banks and develop restructuring plans for those that are financially weak. It will complete ongoing efforts to address problems faced by one large bank with systemic implications. The $\mathrm{BCC}$ will continue strengthening banking supervision by implementing MCM technical assistance recommendations, including by reforming the legal and regulatory framework and reinforcing on-site and off-site inspections.

Reforms to promote private sector development will include advancing the ongoing restructuring of public enterprises, streamlining government regulations, enhancing property rights protection by harmonizing DRC regulations with the OHADA protocol, and adhering to the EITI (MEFP, $₫ 15){ }^{13}$

\section{CAPACITY TO REPAY THE FUND AND RISKS}

\section{The DRC's capacity to repay the Fund is satisfactory, assuming debt relief}

(Tables 5 and 6). After HIPC/MDRI debt relief, outstanding credit to the Fund would decline from SDR 510 million in 2009 to SDR 259 million at end-2010, equivalent to 32 percent of gross international reserves or 5.6 percent of exports of goods and services. The repayment obligations

\footnotetext{
${ }^{13}$ The authorities are working with the World Bank on reforms to reduce the cost of doing business, including by restructuring public enterprises. The DRC's application for adherence to the EITI will be considered in June 2010.
} 
to the Fund would peak at 1.1 percent of exports of goods and services in 2010 and average less than 1 percent in 2010-22.

18. There are risks to program implementation. Re-escalation of conflict in the eastern provinces, deterioration in the terms of trade or spending pressures related to the looming elections could undermine program implementation. Risk of debt distress is expected to remain high even after HIPC/MDRI debt relief. Delayed financial sector reforms could also further weaken the health of the financial sector, while governance concerns could damage the business climate.

\section{Staff Appraisal}

19. The DRC economy is recovering from the adverse effects of the global financial crisis. In 2009, the combined effects of the global economic downturn and lingering conflict in the eastern provinces resulted in a sharp fall in exports, slowdown in economic activity, and uptick in inflation in the context of a steep depreciation of the nominal exchange rate. However, the firming up of world commodity prices is improving export prospects and overall economic growth.

20. The authorities' policy response to the global financial crisis was appropriate. They allowed the fiscal deficit to widen and increased the share of investment in total spending while reducing government borrowing from the central bank. This, together with tight monetary policy, enhanced banking supervision, and liquidity support for weak financial institutions helped mitigate the impact of the global financial crisis on domestic demand, stabilize the exchange rate, ease inflation, and minimize pressures on the financial sector.

\section{Fiscal policy in $\mathbf{2 0 1 0}$ aims at further reducing government recourse to central bank}

financing. Revenue-enhancing measures, together with the impact of higher growth, should help increase the fiscal space for priority programs while reducing fiscal imbalances. Nevertheless, strict adherence to the expenditure commitment and treasury plans will be critical to achieving the program objectives. The authorities' commitment to save a portion of higher projected revenue relative to the original program and the entirety of the second tranche of the SCCA signing bonus is welcome.

22. The gradual easing of monetary policy by the central bank is appropriate. The central bank is committed to keeping its policy rate significantly positive in real terms to avoid the reemergence of pressures on the exchange rate and inflation. Recent measures to enhance coordination between the BCC and the Treasury regarding liquidity management are welcome. The flexible exchange rate regime is appropriate for the DRC economy given its susceptibility to exogenous shocks and its relatively low level of international reserves.

\section{Structural reforms in revenue mobilization and PFM are critical for achieving the}

fiscal policy objectives. The focus on strengthening revenue collection, tightening and rationalizing expenditure commitment procedures, and revamping the legal and regulatory framework of the PFM system is appropriate. 
24. Fiscal decentralization remains a challenge given institutional capacity constraints in PFM at the provincial level. The program appropriately focuses on strengthening such capacity while gradually transferring expenditure responsibilities to individual provinces as capacity is put in place.

25. Financial sector reforms need to be accelerated. The completion of the first wave of on-site inspections of commercial banks is welcome. However, there is a need to develop credible restructuring plans for the weak banks and complete inspections of the remaining banks. Strengthening of the BCC's banking supervision capacity and strict enforcement of its prudential regulations is critical for safeguarding the health of the financial sector.

\section{The authorities need to press ahead with structural reforms to support higher} economic growth. The focus should be on expediting the restructuring of public enterprises that provide growth-critical services (e.g., transport and utilities), adherence to the EITI, and streamlining business regulations.

27. The staff is of the view that the Lending into Arrears Policy should be applied. This is based on the financing assurances review conducted by staff, the good faith efforts exerted by the authorities toward reaching collaborative agreements with commercial creditors, and the fact that relations with commercial creditors would not undermine the program.

28. Staff recommends the completion of the first review of the ECF arrangement. While there are risks that spending overruns related to the looming national elections and security operations could destabilize the economy, the revised program's tighter fiscal policy stance should mitigate such risks. Moreover, donor financial and technical assistance, including for elections and peacemaking efforts, will also help reduce these risks. 
Table 1. Democratic Republic of the Congo: Selected Economic and Financial Indicators, 2007-15

\begin{tabular}{|c|c|c|c|c|c|c|c|c|c|c|c|}
\hline & \multirow[t]{2}{*}{2007} & \multirow{2}{*}{$\begin{array}{l}2008 \\
\text { Prel. }\end{array}$} & \multicolumn{2}{|c|}{2009} & \multicolumn{2}{|c|}{2010} & \multirow{2}{*}{\multicolumn{5}{|c|}{\begin{tabular}{lcc}
2012 & 2013 & 2014 \\
\multicolumn{3}{c}{ Projections }
\end{tabular}}} \\
\hline & & & Prog. & Est. & Prog. & Proj. & & & & & \\
\hline & \multicolumn{11}{|c|}{ (Annual percentage change; unless otherwise indicated) } \\
\hline \multicolumn{12}{|l|}{ GDP and prices } \\
\hline Real GDP & 6.3 & 6.2 & 2.7 & 2.8 & 5.4 & 5.4 & 7.0 & 6.8 & 8.1 & 6.7 & 7.0 \\
\hline GDP deflator & 17.9 & 18.9 & 30.3 & 35.1 & 23.4 & 27.1 & 8.5 & 9.7 & 8.0 & 8.5 & 7.9 \\
\hline Consumer prices, period average & 16.7 & 18.0 & 45.0 & 46.2 & 24.7 & 26.2 & 13.5 & 10.5 & 9.0 & 8.8 & 8.3 \\
\hline Consumer prices, end-of-period & 10.0 & 27.6 & 48.7 & 53.4 & 15.0 & 15.0 & 12.0 & 9.0 & 9.0 & 8.5 & 8.0 \\
\hline \multicolumn{12}{|l|}{ External sector } \\
\hline Exports, f.o.b. (U.S. dollars) & 161.2 & 7.2 & -42.5 & -33.6 & 18.3 & 48.3 & 18.7 & 5.6 & 7.4 & 6.7 & 7.0 \\
\hline Imports, f.o.b. (U.S. dollars) & 81.8 & 27.6 & -21.7 & -26.3 & 21.6 & 46.4 & 16.3 & -0.1 & 7.3 & -1.3 & 5.3 \\
\hline Export volume & 55.5 & 6.3 & 5.4 & 0.6 & 9.4 & 8.0 & 14.4 & 11.9 & 18.0 & 1.8 & -3.8 \\
\hline Import volume & 69.0 & 15.8 & -11.9 & -17.9 & 17.0 & 38.1 & 14.9 & -1.5 & 6.1 & -2.8 & 3.8 \\
\hline Terms of trade & 11.3 & 1.1 & -38.6 & -26.6 & 4.1 & 29.6 & 2.6 & -6.9 & 0.3 & -2.0 & -1.9 \\
\hline Nominal effective exchange rate ${ }^{1}$ & -15.5 & -11.7 & $\ldots$ & -26.7 & $\ldots$ & $\ldots$ & $\ldots$ & $\ldots$ & $\ldots$ & $\ldots \ldots$ & \\
\hline \multirow[t]{2}{*}{ Real effective exchange rate ${ }^{1}$} & -3.1 & -0.7 & $\ldots$ & 1.0 & $\ldots$ & $\ldots$ & & $\ldots$ & & $\ldots \ldots$ & \\
\hline & \multicolumn{11}{|c|}{ (Annual change in percent of beginning-of-period broad money; unless otherwise indicated) } \\
\hline \multicolumn{12}{|l|}{ Money and credit } \\
\hline Broad money & 49.5 & 55.7 & 33.8 & 50.4 & 27.0 & 41.1 & $\ldots$ & $\ldots$ & $\ldots$ & $\ldots$ & $\ldots$ \\
\hline Net foreign assets & 30.8 & -6.0 & -6.9 & 17.0 & 38.6 & 57.8 & $\ldots$ & $\ldots$ & $\ldots$ & $\ldots$ & $\ldots$ \\
\hline Net domestic assets & 20.2 & 65.6 & 41.6 & 33.3 & -11.2 & -16.4 & $\ldots$ & $\ldots$ & $\ldots$ & $\ldots$ & $\ldots$ \\
\hline Domestic credit & 29.5 & 54.7 & 15.6 & 13.5 & 13.8 & 2.1 & $\ldots$ & $\ldots$ & $\ldots$ & $\ldots$ & $\ldots$ \\
\hline \multicolumn{12}{|l|}{ Of which: } \\
\hline Net credit to government & 10.3 & 11.3 & -10.6 & -7.6 & 0.0 & -12.2 & $\ldots$ & $\ldots$ & $\ldots$ & $\ldots$ & $\ldots$ \\
\hline Credit to the private sector (annual percent change) & 73.6 & 141.9 & 55.5 & 41.1 & 25.4 & 36.8 & & & & $\ldots$ & $\ldots$ \\
\hline & & & & (Percent & f GDP; un & s otherwis & dicated) & & & & \\
\hline Central government finance & & & & & & & & & & & \\
\hline Total government revenue & 14.7 & 18.5 & 17.8 & 16.8 & 17.9 & 19.1 & 19.5 & 20.3 & 21.1 & 22.1 & 22.6 \\
\hline Excluding signining bonus from the Sino-Congolese Cooperation Agreement & 14.7 & 18.5 & 15.7 & 15.8 & 17.9 & 17.8 & 19.5 & 20.3 & 21.1 & 22.1 & 22.6 \\
\hline Grants & 2.2 & 2.3 & 9.3 & 6.9 & 6.1 & 10.2 & 9.9 & 8.5 & 6.8 & 5.7 & 5.3 \\
\hline Total government expenditure $^{2}$ & 18.9 & 23.0 & 28.7 & 27.5 & 36.2 & 33.1 & 38.1 & 35.0 & 32.4 & 31.5 & 29.8 \\
\hline Domestic fiscal balance (cash basis) & -0.1 & -0.3 & -3.3 & -2.5 & -1.4 & -1.0 & -0.4 & -0.4 & 0.0 & 0.0 & 0.0 \\
\hline Overall fiscal balance (payment order basis, incl. grants) & -1.9 & -2.2 & -1.6 & -3.8 & -12.2 & -3.7 & -8.7 & -6.3 & -4.6 & -3.7 & -1.9 \\
\hline Overall fiscal balance (cash basis, incl. grants) & -2.5 & -3.0 & -3.0 & -4.8 & -12.7 & -5.1 & -9.6 & -7.0 & -4.7 & -3.7 & -1.9 \\
\hline Investment and saving & & & & & & & & & & & \\
\hline Gross national saving & 17.1 & 6.1 & 7.0 & 9.3 & 5.8 & 10.4 & 19.2 & 15.6 & 17.1 & 22.0 & 21.7 \\
\hline Government & -1.2 & -0.8 & 1.0 & -1.3 & -0.1 & 1.8 & 2.8 & 3.2 & 3.6 & 4.2 & 4.5 \\
\hline Nongovernment & 18.3 & 6.9 & 6.0 & 10.5 & 5.9 & 8.7 & 16.4 & 12.4 & 13.5 & 17.8 & 17.2 \\
\hline Investment & 18.2 & 22.0 & 23.4 & 19.4 & 33.5 & 30.4 & 40.7 & 33.6 & 30.7 & 29.8 & 28.5 \\
\hline Government $^{3}$ & 2.3 & 3.7 & 9.3 & 7.8 & 18.0 & 14.7 & 21.1 & 17.7 & 14.7 & 13.3 & 11.5 \\
\hline Nongovernment & 15.9 & 18.2 & 14.1 & 11.6 & 15.5 & 15.7 & 19.6 & 15.9 & 16.0 & 16.5 & 17.0 \\
\hline Balance of payments & & & & & & & & & & & \\
\hline Exports of goods and services & 65.2 & 61.3 & 41.8 & 45.2 & 42.7 & 56.1 & 63.1 & 61.6 & 61.3 & 60.4 & 59.5 \\
\hline Imports of goods and services & 68.6 & 76.4 & 66.4 & 60.9 & 71.1 & 75.1 & 83.0 & 77.4 & 76.7 & 69.8 & 67.7 \\
\hline Current account balance, incl. transfers & -1.1 & -15.9 & -16.4 & -10.1 & -27.7 & -20.0 & -21.5 & -18.0 & -13.6 & -7.9 & -6.8 \\
\hline Current account balance, excl. transfers & -9.7 & -26.5 & -30.4 & -22.7 & -34.4 & -27.0 & -28.1 & -24.2 & -19.7 & -13.9 & -12.7 \\
\hline Current account balance, incl. transfers, after debt relief & 0.5 & -14.3 & -13.0 & -6.1 & -24.1 & -19.4 & -21.5 & -18.0 & -13.6 & -7.9 & -6.8 \\
\hline Gross official reserves (end-of-period, millions of U.S. dollars) & 181 & 78 & 1,039 & 999 & 1,223 & 1,257 & 1,359 & 1,533 & 1,927 & 2,080 & 2,360 \\
\hline Gross official reserves (weeks of nonaid-related imports of goods and services) & 1.2 & 0.8 & 9.9 & 7.2 & 8.7 & 7.5 & 7.9 & 8.0 & 10.1 & 10.1 & 11.1 \\
\hline & & & & Millions of & S. dollars; & less othe & indicated & & & & \\
\hline External public debt & & & & & & & & & & & \\
\hline Total stock, including IMF & 12,929 & 13,532 & 12,785 & 13,705 & 4,329 & 2,931 & 4,404 & 5,662 & 6,550 & 7,381 & 7,944 \\
\hline Present value (PV) of debt ${ }^{4}$ & $\ldots$ & $\ldots \ldots$ & $\ldots$ & 12,025 & $\ldots$ & 3,773 & 5,045 & 6,067 & 6,814 & 6,594 & 6,832 \\
\hline PV of debt (percent of exports of goods and services) ${ }^{4}$ & 0.0 . & & & 193.3 & & 58.9 & 74.0 & 75.2 & 76.8 & 69.4 & 67.0 \\
\hline Scheduled debt service & 916.8 & 999.5 & 815.5 & $1,062.6$ & 252.1 & 203.4 & 87.3 & 102.1 & 120.4 & 133.5 & 169.5 \\
\hline Percent of exports of goods and services & 14.0 & 12.0 & 18.2 & 21.2 & 4.9 & 2.9 & 1.0 & 1.2 & 1.3 & 1.3 & 1.6 \\
\hline Percent of government revenue & 53.9 & 41.5 & 29.4 & 40.3 & 8.8 & 5.5 & 2.3 & 2.5 & 2.8 & 2.9 & 3.3 \\
\hline Exchange rate, (CGF per U.S. dollar) & & & & & & & & & & & \\
\hline Period average & 516 & 563 & $\ldots$ & 817 & & $\ldots$ & $\ldots$ & $\ldots$ & $\ldots$ & $\ldots \ldots$ & \\
\hline End-of-period & 503 & 639 & $\ldots$ & 903 & & $\ldots$ & $\cdots$ & $\ldots$ & $\ldots$ & $\ldots \ldots$ & \\
\hline Memorandum item: & & & & & & & & & & & \\
\hline Nominal GDP (CGF billions) & 5,175 & 6,530 & 8,649 & 9,073 & 11,361 & 12,163 & 14,125 & 16,538 & 19,295 & 22,323 & 25,766 \\
\hline
\end{tabular}

Sources: Congolese authorities and IMF staff estimates and projections.

${ }^{1}$ Change in annual average. Minus sign indicates depreciation.

${ }^{2}$ Includes interest due before debt relief and expenditure financed by HIPC resources.

${ }^{3}$ Includes investment financed by resources released under the enhanced HIPC Initiative.

${ }^{4}$ Estimates and projections are based on calculations under the 2010 HIPC Debt Sustainability Analysis (www.imf.org). Includes assistance beyond

the terms of the enhanced HIPC Initiative granted by some Paris Club creditors. Exports are on a three-year backward moving average; projections

assume DRC reaches the HIPC completion point by end-June 2010. 
Table 2a. Democratic Republic of the Congo: Central Government Financial Operations, 2007-12

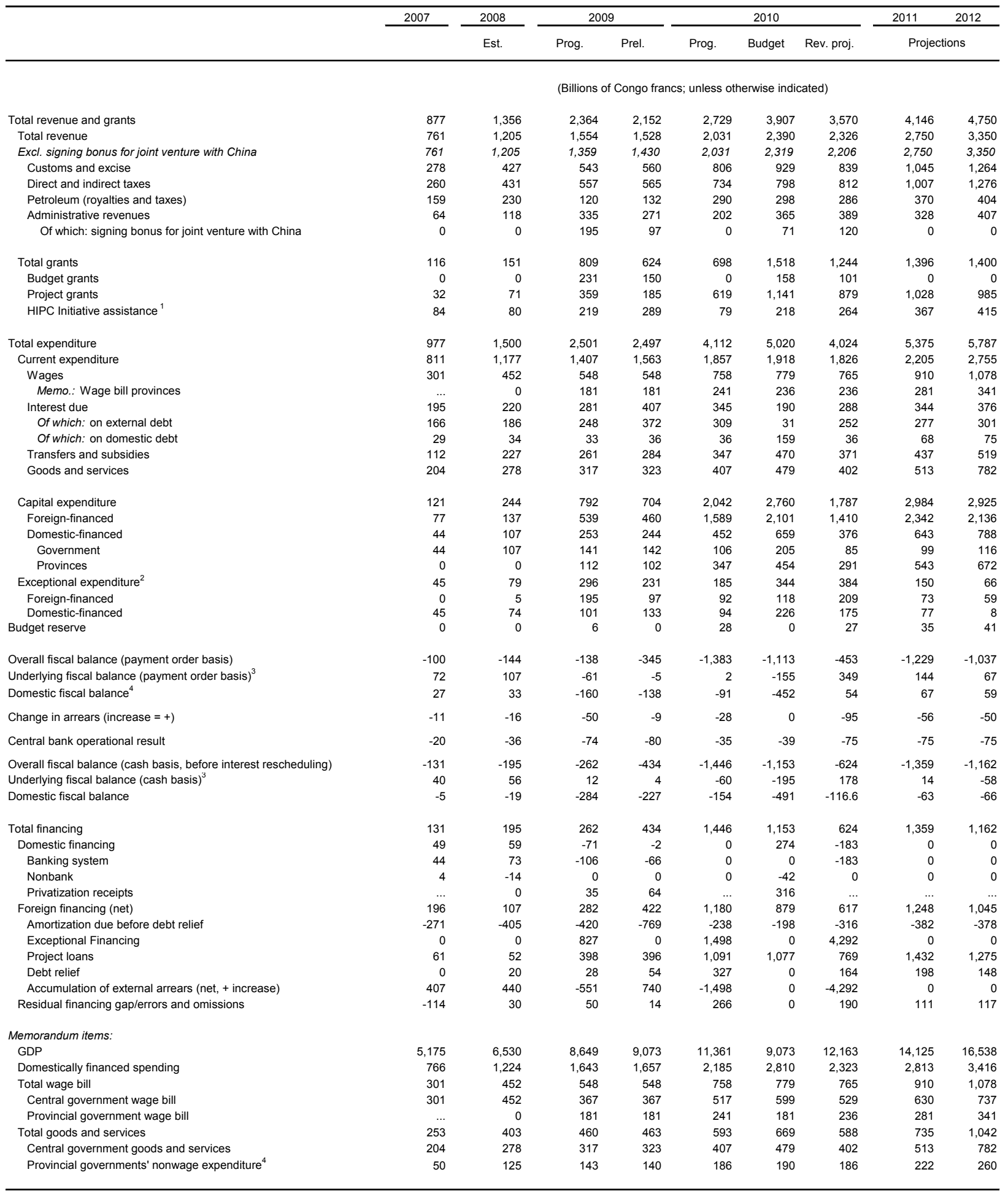

Sources: Congolese authorities and IMF staff estimates and projections.

${ }^{1}$ Reflects revised calculation of HIPC Initiative assistance on the basis of the 2010 Debt Sustainability Analysis (www.imf.org).

${ }^{2}$ Exceptional expenditure includes spending for the Demobilization, Disarmament, and Reintegration (DDR) program, and cost of the elections.

${ }^{3}$ Underlying fiscal balance is defined as revenue minus expenditure and excluding interest on foreign debt, foreign-financed capital expenditure, all exceptional spending, and repayment of domestic arrears.

${ }^{4}$ The domestic fiscal balance is defined as revenue (excluding the signing bonus from the SCCA) minus total expenditure (excluding interest on foreign debt, foreign-financed capital and exceptional expenditure)

${ }^{5}$ The domestic fiscal balance (cash basis) is defined as domestic fiscal balance (payment order basis) minus BCC's operating deficit minus the net repayment of domestic arrears. 
Table 2b. Democratic Republic of the Congo: Central Government Financial Operations, 2007-12

\begin{tabular}{|c|c|c|c|c|c|c|c|c|c|c|c|}
\hline & \multirow{2}{*}{$\begin{array}{l}2007 \\
\text { Est. }\end{array}$} & \multirow{2}{*}{$\begin{array}{l}2008 \\
\text { Est. }\end{array}$} & \multicolumn{3}{|c|}{2009} & \multicolumn{4}{|c|}{2010} & 2011 & \multirow[t]{2}{*}{2012} \\
\hline & & & $\begin{array}{l}\text { Program } \\
\text { IMF Country } \\
\text { Report No. } \\
10 / 88\end{array}$ & $\begin{array}{c}\text { Program } \\
\text { Revised GDP }\end{array}$ & Est. & $\begin{array}{l}\text { Program } \\
\text { IMF Country } \\
\text { Report No. } \\
10 / 88\end{array}$ & $\begin{array}{c}\text { Program } \\
\text { Revised GDP }\end{array}$ & Budget & Proj. & Projectio & \\
\hline & \multicolumn{11}{|c|}{ (Percent of GDP; unless otherwise indicated) } \\
\hline Total revenue and grants & 17.0 & 20.8 & 27.3 & 26.1 & 23.7 & 24.0 & 22.4 & 34.4 & 29.4 & 29.4 & 28.7 \\
\hline Total revenue & 14.7 & 18.5 & 18.0 & 17.1 & 16.8 & 17.9 & 16.7 & 21.0 & 19.1 & 19.5 & 20.3 \\
\hline Excl. signing bonus for joint venture with China & & $\ldots$ & 15.7 & 15.0 & 15.8 & 17.9 & 16.7 & 21.0 & 18.1 & $\ldots$ & \\
\hline Customs and excise & 5.4 & 6.5 & 6.3 & 6.0 & 6.2 & 7.1 & 6.6 & 8.2 & 6.9 & 7.4 & 7.6 \\
\hline Direct and indirect taxes & 5.0 & 6.6 & 6.4 & 6.1 & 6.2 & 6.5 & 6.0 & 7.0 & 6.7 & 7.1 & 7.7 \\
\hline Petroleum (royalties and taxes) & 3.1 & 3.5 & 1.4 & 1.3 & 1.5 & 2.6 & 2.4 & 2.6 & 2.4 & 2.6 & 2.4 \\
\hline Other & 1.2 & 1.8 & 3.9 & 3.7 & 3.0 & 1.8 & 1.7 & 3.2 & 3.2 & 2.3 & 2.5 \\
\hline Of which: Signing bonus from joint venture with China & $\ldots$ & 0.0 & 2.3 & 2.2 & 1.1 & $\ldots$ & $\ldots$ & $\ldots$ & 1.0 & $\ldots$ & $\ldots$ \\
\hline Total grants & 2.2 & 2.3 & 9.4 & 8.9 & 6.9 & 6.1 & 5.7 & 13.4 & 10.2 & 9.9 & 8.5 \\
\hline Budget grants & 0.0 & 0.0 & 2.7 & 2.5 & 1.7 & 0.0 & 0.0 & 1.4 & 0.8 & 0.0 & 0.0 \\
\hline Project grants & 0.6 & 1.1 & 4.2 & 4.0 & 2.0 & 5.4 & 5.1 & 10.0 & 7.2 & 7.3 & 6.0 \\
\hline HIPC Initiative assistance ${ }^{1}$ & 1.6 & 1.2 & 2.5 & 2.4 & 3.2 & 0.7 & 0.7 & 1.9 & 2.2 & 2.6 & 2.5 \\
\hline Total expenditure & 18.9 & 23.0 & 28.9 & 27.6 & 27.5 & 36.2 & 33.8 & 44.2 & 33.1 & 38.1 & 35.0 \\
\hline Current expenditure & 15.7 & 18.0 & 16.3 & 15.5 & 17.2 & 16.3 & 15.3 & 16.9 & 15.0 & 15.6 & 16.7 \\
\hline Wages & 5.8 & 6.9 & 6.3 & 6.0 & 6.0 & 6.7 & 6.2 & 6.9 & 6.3 & 6.4 & 6.5 \\
\hline Interest due & 3.8 & 3.4 & 3.3 & 3.1 & 4.5 & 3.0 & 2.8 & 1.7 & 2.4 & 2.4 & 2.3 \\
\hline Of which: On external debt & 3.2 & 2.9 & 2.9 & 2.7 & 4.1 & 2.7 & 2.5 & 0.3 & 2.1 & 2.0 & 1.8 \\
\hline Of which: on domestic debt & 0.6 & 0.5 & 0.4 & 0.4 & 0.4 & 0.3 & 0.3 & 1.4 & 0.3 & 0.5 & 0.5 \\
\hline Transfers and subsidies & 2.2 & 3.5 & 3.0 & 2.9 & 3.1 & 3.1 & 2.9 & 4.1 & 3.0 & 3.1 & 3.1 \\
\hline Goods and servicees & 3.9 & 4.3 & 3.7 & 3.5 & 3.6 & 3.6 & 3.3 & 4.2 & 3.3 & 3.6 & 4.7 \\
\hline Of which: Institutions & 0.9 & 1.2 & 0.9 & 0.9 & 0.8 & 0.9 & 0.8 & 1.5 & 0.9 & 0.9 & 1.0 \\
\hline Ministries & 2.8 & 2.5 & 2.1 & 2.0 & 2.1 & 1.9 & 1.8 & 2.0 & 1.7 & 2.0 & 3.0 \\
\hline Centralized payments & 0.3 & 0.5 & 0.7 & 0.6 & 0.7 & 0.7 & 0.7 & 0.7 & 0.7 & 0.7 & 0.7 \\
\hline Memo.: Transferred to provinces & $\ldots$ & 1.9 & 0.0 & 0.6 & 0.0 & -0.5 & -0.5 & 0.0 & 0.0 & 0.0 & 0.0 \\
\hline Total goods and services & $\ldots$ & 6.2 & 0.0 & 0.0 & 3.6 & 3.1 & 2.9 & 4.2 & 3.3 & 3.6 & 4.7 \\
\hline Capital expenditure & 2.3 & 3.7 & 9.2 & 8.7 & 7.8 & 18.0 & 16.8 & 24.3 & 14.7 & 21.1 & 17.7 \\
\hline Foreign-financed & 1.5 & 2.1 & 6.2 & 5.9 & 5.1 & 14.0 & 13.1 & 18.5 & 11.6 & 16.6 & 12.9 \\
\hline Domestic-financed & 0.9 & 1.6 & 2.9 & 2.8 & 2.7 & 4.0 & 3.7 & 5.8 & 3.1 & 4.5 & 4.8 \\
\hline Government & 0.9 & 1.6 & 1.6 & 1.6 & 1.6 & 0.9 & 0.9 & 1.8 & 0.7 & 0.7 & 0.7 \\
\hline Provinces & 0.0 & 0.0 & 1.3 & 1.2 & 1.1 & 3.1 & 2.9 & 4.0 & 2.4 & 3.8 & 4.1 \\
\hline Exceptional expenditure $^{2}$ & 0.9 & 1.2 & 3.4 & 3.3 & 2.5 & 1.6 & 1.5 & 3.0 & 3.2 & 1.1 & 0.4 \\
\hline Foreign-financed & 0.0 & 0.1 & 2.3 & 2.1 & 1.1 & 0.8 & 0.8 & 1.0 & 1.7 & 0.5 & 0.4 \\
\hline Domestic-financed & 0.9 & 1.1 & 1.2 & 1.1 & 1.5 & 0.8 & 0.8 & 2.0 & 1.4 & 0.5 & 0.0 \\
\hline Budget reserve & 0.0 & 0.0 & 0.1 & 0.1 & 0.0 & 0.3 & 0.3 & 0.0 & 0.2 & 0.3 & 0.3 \\
\hline Overall fiscal balance (payment order basis) & -1.9 & -2.2 & -1.6 & -1.5 & -3.8 & -12.2 & -11.4 & -9.8 & -3.7 & -8.7 & -6.3 \\
\hline Underlying fiscal balance (payment order basis) $)^{3}$ & 1.4 & 1.6 & -0.7 & -0.7 & 0.0 & 0.0 & 0.0 & -1.4 & 2.9 & 1.0 & 0.4 \\
\hline Domestic fiscal balance $^{3}$ & 0.5 & 0.5 & -1.9 & -1.8 & -1.5 & -0.8 & -0.8 & -4.0 & 0.4 & 0.5 & 0.4 \\
\hline Change in arrears (increase $=+$ ) & -0.2 & -0.2 & -0.6 & -0.6 & -0.1 & -0.2 & -0.2 & 0.0 & -0.8 & -0.4 & -0.3 \\
\hline Central bank operational result & -0.4 & -0.5 & -0.9 & -0.8 & -0.9 & -0.3 & -0.3 & -0.3 & -0.6 & -0.5 & -0.5 \\
\hline Overall fiscal balance (cash basis, before interest rescheduling) & -2.5 & -3.0 & -3.0 & -2.9 & -4.8 & -12.7 & -11.9 & -10.1 & -5.1 & -9.6 & -7.0 \\
\hline Underlying fiscal balance (cash basis) ${ }^{4}$ & 0.8 & 0.9 & 0.1 & 0.1 & 0.0 & -0.5 & -0.5 & -1.7 & 1.5 & 0.1 & -0.4 \\
\hline Domestic fiscal balance & -0.1 & -0.3 & -3.3 & -3.1 & -2.5 & -1.4 & -1.3 & -4.3 & -1.0 & -0.4 & -0.4 \\
\hline Total financing & 2.5 & 3.0 & 3.0 & 2.9 & 4.8 & 12.7 & 11.9 & 10.1 & 5.1 & 9.6 & 7.0 \\
\hline Domestic financing & 0.9 & 0.9 & -0.8 & -0.8 & 0.0 & 0.0 & 0.0 & 2.4 & -1.5 & 0.0 & 0.0 \\
\hline Banking system & 0.9 & 1.1 & -1.2 & -1.2 & -0.7 & 0.0 & 0.0 & 0.0 & -1.5 & 0.0 & 0.0 \\
\hline Foreign financing (net) & 3.8 & 1.6 & 3.3 & 3.1 & 4.6 & 10.4 & 9.7 & 7.7 & 5.1 & 8.8 & 6.3 \\
\hline Residual financing gap & -2.2 & 0.5 & 0.6 & 0.6 & 0.2 & 2.3 & 2.2 & 0.0 & 1.6 & 0.8 & 0.7 \\
\hline \multicolumn{12}{|l|}{ Memorandum items: } \\
\hline GDP (billions of CGF) & 5,175 & 6,530 & 8,649 & 9,073 & 9,073 & 11,361 & 12,163 & 11,366 & 12,163 & 14,125 & 16,538 \\
\hline Domestically financed spending & 14.8 & 18.7 & 19.0 & 18.1 & 18.3 & 19.2 & 18.0 & 24.7 & 19.1 & 19.9 & 20.7 \\
\hline Total wage bill & 5.8 & 6.9 & 6.3 & 6.0 & 6.0 & 6.7 & 6.2 & 6.9 & 6.3 & 6.4 & 6.5 \\
\hline Central government wage bill & 5.8 & 6.9 & 4.2 & 4.0 & 4.0 & 4.5 & 4.2 & 5.3 & 4.3 & 4.5 & 4.5 \\
\hline Provincial government wage bill & $\ldots$ & $\ldots$ & 2.1 & 2.0 & 2.0 & 2.1 & 2.0 & 1.6 & 1.9 & 2.0 & 2.1 \\
\hline Total goods and services & 4.9 & 6.2 & 5.3 & 5.1 & 5.1 & 5.2 & 4.9 & 5.9 & 4.8 & 5.2 & 6.3 \\
\hline Central government goods and services & 3.9 & 4.3 & 3.7 & 3.5 & 3.6 & 3.6 & 3.3 & 4.2 & 3.3 & 3.6 & 4.7 \\
\hline Provincial governments' nonwage expenditure & 1.0 & 1.9 & 1.6 & 1.6 & 1.5 & 1.6 & 1.5 & 1.7 & 1.5 & 1.6 & 1.6 \\
\hline
\end{tabular}

Sources: Congolese authorities and IMF staff estimates and projections.

'Reflects revised calculation of HIPC Initiative assistance on the basis of 2010 Debt Sustainability Analysis (www.imf.org).

2Exceptional expenditure includes spending for the Demobilization, Disarmament, and Reintegration (DDR) program, and cost of the elections.

${ }^{3}$ The domestic fiscal balance is defined as revenue (excluding the signing bonus from the SCCA) minus total expenditure

(excluding interest on foreign debt, foreign-financed capital and exceptional expenditure).

Underlying fiscal balance is defined as revenue minus expenditure and excluding interest on foreign debt, foreign-financed capital expenditure,

all exceptional spending, and repayment of domestic arrears.

${ }^{5}$ The domestic fiscal balance (cash basis) is defined as domestic fiscal balance (payment order basis) minus BCC's operating deficit minus the net repayment of domestic arrears. 
Table 3a. Democratic Republic of the Congo: Monetary Survey, 2007-10 (At current exchange rates)

\begin{tabular}{|c|c|c|c|c|c|c|}
\hline & \multirow{2}{*}{$\frac{2007}{\mathrm{Dec}}$} & \multirow{2}{*}{$\frac{2008}{\mathrm{Dec}}$} & \multicolumn{2}{|c|}{2009} & \multicolumn{2}{|c|}{2010} \\
\hline & & & $\begin{array}{l}\text { Dec } \\
\text { Prog. }\end{array}$ & $\begin{array}{l}\text { Dec } \\
\text { Prel }\end{array}$ & $\begin{array}{l}\text { Mar } \\
\text { Prel }\end{array}$ & $\begin{array}{c}\text { Dec } \\
\text { Rev Proj }\end{array}$ \\
\hline & \multicolumn{6}{|c|}{ (Billions of Congo francs) } \\
\hline Net foreign assets & -172.6 & -211.0 & -279.7 & -41.0 & 119.7 & 827.1 \\
\hline Net domestic assets & 831.4 & $1,252.4$ & $1,668.2$ & $1,584.5$ & $1,477.4$ & $1,338.2$ \\
\hline Domestic credit & 373.4 & 724.3 & 880.3 & 859.4 & 735.3 & 891.0 \\
\hline Net credit to government & 176.2 & 248.9 & 143.1 & 172.8 & 58.3 & -10.3 \\
\hline Credit to the private sector & 195.2 & 472.3 & 734.1 & 657.7 & 652.0 & 899.6 \\
\hline Credit to parastatals & 1.9 & 3.1 & 3.1 & 28.9 & 25.0 & 1.7 \\
\hline Other items, net (including valuation change) & 458.1 & 528.1 & 787.8 & 725.0 & 742.1 & 447.1 \\
\hline Broad money (M2) & 641.2 & 998.4 & $1,335.5$ & $1,501.9$ & $1,553.4$ & $2,118.6$ \\
\hline Narrow money (M1) & 300.3 & 392.5 & 436.8 & 479.9 & 454.3 & 601.6 \\
\hline Currency in circulation & 233.3 & 304.6 & 354.5 & 381.5 & 353.8 & 475.3 \\
\hline Demand deposits & 67.0 & 87.9 & 82.3 & 98.4 & 100.5 & 126.3 \\
\hline Quasi money & 341.0 & 606.0 & 898.8 & $1,022.0$ & $1,099.1$ & $1,517.0$ \\
\hline Time deposits in domestic currency & 2.5 & 1.3 & 4.7 & 3.5 & 4.7 & 5.0 \\
\hline Foreign currency deposits & 338.5 & 604.7 & 894.0 & $1,018.5$ & $1,094.4$ & $1,512.0$ \\
\hline \multirow[t]{2}{*}{ Import deposits } & 17.6 & 42.9 & 52.9 & 41.7 & 43.6 & 46.7 \\
\hline & \multicolumn{6}{|c|}{ (Year-on year change in percent) } \\
\hline Net foreign assets & 43.4 & -22.2 & -32.6 & 80.6 & 142.9 & $2,119.5$ \\
\hline Net domestic assets & 11.6 & 50.6 & 33.2 & 26.5 & 3.0 & -15.5 \\
\hline Domestic credit & 51.3 & 94.0 & 21.5 & 18.7 & -10.5 & 3.7 \\
\hline Net credit to government & 33.4 & 41.2 & -42.5 & -30.6 & -73.4 & -106.0 \\
\hline Credit to the private sector & 73.6 & 141.9 & 55.5 & 39.3 & 9.0 & 36.8 \\
\hline Credit to parastatals & -11.8 & 63.4 & 0.0 & 823.4 & 516.8 & -94.1 \\
\hline Other items, net (including valuation change) & -8.1 & 15.3 & 49.2 & 37.3 & 21.2 & -38.3 \\
\hline Broad money (M2) & 49.5 & 55.7 & 33.8 & 50.4 & 40.3 & 41.1 \\
\hline Narrow money (M1) & 41.2 & 30.7 & 11.3 & 22.3 & 23.0 & 25.4 \\
\hline Currency in circulation & 28.2 & 30.6 & 16.4 & 25.3 & 21.1 & 24.6 \\
\hline Demand deposits & 117.6 & 31.2 & -6.4 & 11.9 & 30.1 & 28.4 \\
\hline Quasi money & 57.8 & 77.7 & 48.3 & 68.7 & 49.0 & 48.4 \\
\hline Time deposits in domestic currency & 483.0 & -47.8 & 262.0 & 167.9 & 171.6 & 42.0 \\
\hline Foreign currency deposits & 56.9 & 78.6 & 47.9 & 68.4 & 48.7 & 48.5 \\
\hline \multirow[t]{2}{*}{ Import deposits } & 54.0 & 144.0 & 23.3 & -2.9 & -7.5 & 12.0 \\
\hline & \multicolumn{6}{|c|}{ (Annual change in percent of beginning-of-period broad money) } \\
\hline Net foreign assets & 30.8 & -6.0 & -6.9 & 17.0 & 10.7 & 57.8 \\
\hline Net domestic assets & 20.2 & 65.6 & 41.6 & 33.3 & -7.1 & -16.4 \\
\hline Domestic credit & 29.5 & 54.7 & 15.6 & 13.5 & -8.3 & 2.1 \\
\hline Net credit to government & 10.3 & 11.3 & -10.6 & -7.6 & -7.6 & -12.2 \\
\hline Credit to the private sector & 19.3 & 43.2 & 26.2 & 18.6 & -0.4 & 16.1 \\
\hline Credit to parastatals & -0.1 & 0.2 & 0.0 & 2.6 & -0.3 & -1.8 \\
\hline Other items, net (including valuation change) & -9.4 & 10.9 & 26.0 & 19.7 & 1.1 & -18.5 \\
\hline Broad money (M2) & 49.5 & 55.7 & 33.8 & 50.4 & 3.4 & 41.1 \\
\hline Narrow money (M1) & 20.4 & 14.4 & 4.4 & 8.8 & -1.7 & 8.1 \\
\hline Currency in circulation & 12.0 & 11.1 & 5.0 & 7.7 & -1.8 & 6.2 \\
\hline Demand deposits & 8.4 & 3.3 & -0.6 & 1.0 & 0.1 & 1.9 \\
\hline Quasi money & 29.1 & 41.3 & 29.3 & 41.7 & 5.1 & 33.0 \\
\hline Time deposits in domestic currency & 0.5 & -0.2 & 0.3 & 0.2 & 0.1 & 0.1 \\
\hline Foreign currency deposits & 28.6 & 41.5 & 29.0 & 41.4 & 5.1 & 32.9 \\
\hline Import deposits & 1.4 & 4.0 & 1.0 & -0.1 & 0.1 & 0.3 \\
\hline \multicolumn{7}{|l|}{ Memorandum items: } \\
\hline Nominal GDP (billions of Congo francs) & $5,174.7$ & $6,529.9$ & $8,729.0$ & $9,072.8$ & $\ldots$ & $12,163.0$ \\
\hline Velocity (GDP/broad money) & 8.1 & 6.5 & 6.5 & 6.0 & $\ldots$ & 5.7 \\
\hline Foreign currency deposits (percent of M2) & 52.8 & 60.6 & 66.9 & 67.8 & 70.5 & 71.4 \\
\hline Foreign currency deposits (percent of total deposit & 83.0 & 87.1 & 91.1 & 90.9 & 91.2 & 92.0 \\
\hline
\end{tabular}

Sources: Congolese authorities and IMF staff estimates and projections. 
Table 3b. Democratic Republic of the Congo: Accounts of the Central Bank of the Congo, 2007-10 (At current exchange rates)

\begin{tabular}{|c|c|c|c|c|c|c|}
\hline & \multirow{2}{*}{$\begin{array}{r}2007 \\
\text { Dec }\end{array}$} & \multirow{2}{*}{$\frac{2008}{\mathrm{Dec}}$} & \multicolumn{2}{|c|}{2009} & \multicolumn{2}{|c|}{2010} \\
\hline & & & $\begin{array}{l}\text { Dec } \\
\text { Prog. }\end{array}$ & $\begin{array}{l}\text { Dec } \\
\text { Prel }\end{array}$ & $\begin{array}{l}\text { Mar } \\
\text { Prel }\end{array}$ & $\begin{array}{r}\text { Dec } \\
\text { Rev Proj }\end{array}$ \\
\hline & \multicolumn{6}{|c|}{ (Billions of Congo francs) } \\
\hline Net foreign assets & -333.3 & -390.1 & -515.4 & -557.6 & -520.2 & 38.1 \\
\hline Net domestic assets & 621.7 & 773.8 & 974.2 & 1036.9 & 1007.1 & 589.8 \\
\hline Domestic credit & 206.6 & 295.2 & 230.5 & 274.8 & 229.6 & 102.2 \\
\hline Net credit to government & 198.7 & 272.4 & 166.6 & 200.3 & 121.7 & 17.1 \\
\hline Credit to the private sector & 3.485 & 3.0 & 3.0 & 3.6 & 3.6 & 3.6 \\
\hline Credit to parastatals & 0.0 & 0.0 & 0.0 & 0.0 & 0.0 & 0.0 \\
\hline Claims on deposit money banks & 4.4 & 19.7 & 60.9 & 70.9 & 104.2 & 81.5 \\
\hline Other items, net & 415.1 & 478.6 & 743.7 & 762.1 & 777.5 & 487.6 \\
\hline Of which: BTR & 32.9 & 47.0 & 81.9 & 56.7 & 28.8 & 56.4 \\
\hline Base money & 288.5 & 383.7 & 458.7 & 479.2 & 486.9 & 627.8 \\
\hline Narrow base money & 283.6 & 378.1 & 453.1 & 471.7 & 479.1 & 620.3 \\
\hline Currency in circulation & 243.8 & 320.3 & 367.5 & 394.7 & 364.9 & 488.8 \\
\hline In bank vaults & 10.5 & 15.7 & 13.0 & 13.2 & 11.1 & 13.5 \\
\hline Outside banks & 233.3 & 304.6 & 354.5 & 381.5 & 353.8 & 475.3 \\
\hline Deposits at the central bank & 38.7 & 56.2 & 84.1 & 69.7 & 111.6 & 124.1 \\
\hline Private sector deposits & 0.6 & 0.9 & 0.9 & 4.9 & 1.4 & 4.9 \\
\hline Parastatal deposits & 0.4 & 0.7 & 0.7 & 2.4 & 1.2 & 2.4 \\
\hline Foreign currency deposits & 1.9 & 2.7 & 2.7 & 3.4 & 3.9 & 3.4 \\
\hline \multirow[t]{2}{*}{ Import deposits } & 3.0 & 2.9 & 2.9 & 4.2 & 3.9 & 4.2 \\
\hline & \multicolumn{6}{|c|}{ (Year-on year change in percent) } \\
\hline Net foreign assets & 23.1 & -17.0 & -32.1 & -43.0 & -6.5 & 106.8 \\
\hline Net domestic assets & -3.6 & 24.5 & 25.9 & 34.0 & 17.6 & -43.1 \\
\hline Domestic credit & 15.5 & 42.9 & -21.9 & -6.9 & -30.4 & -62.8 \\
\hline Net credit to government & 21.8 & 37.1 & -38.8 & -26.5 & -51.6 & -91.5 \\
\hline Credit to the private sector & 279.1 & -13.1 & 0.0 & 17.5 & -25.1 & 0.0 \\
\hline Credit to parastatals & -48.5 & 0.0 & 0.0 & 0.0 & 0.0 & 0.0 \\
\hline Claims on deposit money banks & -70.2 & 347.7 & 208.7 & 259.9 & 41.8 & 14.9 \\
\hline Other items, net & -10.9 & 15.3 & 55.4 & 59.2 & 47.6 & -36.0 \\
\hline Base money & 36.5 & 33.0 & 19.6 & 24.9 & 32.3 & 31.0 \\
\hline Narrow base money & 38.9 & 33.3 & 19.8 & 24.8 & 32.7 & 31.5 \\
\hline Currency in circulation & 31.5 & 31.4 & 14.7 & 23.2 & 14.5 & 23.9 \\
\hline In bank vaults & 205.4 & 49.1 & -17.3 & -16.1 & -58.1 & 2.4 \\
\hline Outside banks & 28.2 & 30.6 & 16.4 & 25.3 & 21.1 & 24.6 \\
\hline Deposits at the central bank & 110.4 & 45.2 & 49.5 & 24.0 & 170.4 & 78.1 \\
\hline Private sector deposits & 426.3 & 48.5 & 0.0 & 441.1 & 942.3 & 0.0 \\
\hline Parastatal deposits & 101.8 & 62.6 & 0.0 & 255.1 & 35.0 & 0.0 \\
\hline Foreign currency deposits & -56.0 & 39.0 & 0.0 & 24.5 & 15.7 & 0.0 \\
\hline \multirow[t]{2}{*}{ Import deposits } & 5.0 & -1.7 & 0.0 & 42.8 & 9.0 & 0.0 \\
\hline & \multicolumn{6}{|c|}{ (Annual change in percent of beginning-of-period base money) } \\
\hline Net foreign assets & 47.5 & -19.7 & -32.7 & -43.7 & 7.8 & 124.3 \\
\hline Net domestic assets & -11.0 & 52.7 & 52.2 & 68.6 & -6.2 & -93.3 \\
\hline Domestic credit & 13.2 & 30.7 & -16.9 & -5.3 & -9.4 & -36.0 \\
\hline Net credit to government & 16.8 & 25.6 & -27.6 & -18.8 & -16.4 & -38.2 \\
\hline Credit to the private sector & 1.2 & -0.2 & 0.0 & 0.1 & 0.0 & 0.0 \\
\hline Credit to parastatals & 0.0 & 0.0 & 0.0 & 0.0 & 0.0 & 0.0 \\
\hline Claims on deposit money banks & -4.9 & 5.3 & 10.7 & 13.4 & 6.9 & 2.2 \\
\hline Other items, net & -24.1 & 22.0 & 69.1 & 73.9 & 3.2 & -57.3 \\
\hline Base money & 36.5 & 33.0 & 19.6 & 24.9 & 1.6 & 31.0 \\
\hline Narrow base money & 37.6 & 32.8 & 19.6 & 24.4 & 1.5 & 31.0 \\
\hline Currency in circulation & 27.7 & 26.5 & 12.3 & 19.4 & -6.2 & 19.6 \\
\hline In bank vaults & 3.4 & 1.8 & -0.7 & -0.7 & -0.4 & 0.1 \\
\hline Outside banks & 24.3 & 24.7 & 13.0 & 20.0 & -5.8 & 19.6 \\
\hline Deposits at the central bank & 9.6 & 6.1 & 7.3 & 3.5 & 8.7 & 11.4 \\
\hline Private sector deposits & 0.2 & 0.1 & 0.0 & 1.1 & -0.7 & 0.0 \\
\hline Parastatal deposits & 0.1 & 0.1 & 0.0 & 0.5 & -0.3 & 0.0 \\
\hline Foreign currency deposits & -1.2 & 0.3 & 0.0 & 0.2 & 0.1 & 0.0 \\
\hline Import deposits & 0.1 & 0.0 & 0.0 & 0.3 & -0.1 & 0.0 \\
\hline
\end{tabular}

Sources: Congolese authorities and IMF staff estimates and projections. 
Table 4. Democratic Republic of the Congo: Balance of Payments Summary, 2008-15

\begin{tabular}{|c|c|c|c|c|c|c|c|c|}
\hline & 2008 & 2009 & 2010 & 2011 & 2012 & 2013 & 2014 & 2015 \\
\hline & & Prel. & \multicolumn{6}{|c|}{ Projections } \\
\hline & \multicolumn{8}{|c|}{ (Millions of dollars; unless otherwise indicated) } \\
\hline Current account & $-1,839$ & $-1,123$ & $-2,529$ & $-2,832$ & $-2,559$ & $-2,115$ & $-1,325$ & $-1,245$ \\
\hline Merchandise trade & -125 & -578 & -764 & -730 & -287 & -297 & 412 & 587 \\
\hline $\begin{array}{l}\text { Exports, f.o.b. } \\
\text { Of which: mining products }\end{array}$ & 6,585 & 4,371 & 6,483 & 7,695 & 8,127 & 8,730 & 9,319 & 9,969 \\
\hline Imports, f.o.b. & $-6,711$ & $-4,949$ & $-7,247$ & $-8,425$ & $-8,414$ & $-9,028$ & $-8,907$ & $-9,382$ \\
\hline Of which: aid-related imports & $-1,109$ & $-1,329$ & $-1,687$ & $-1,740$ & $-1,645$ & $-1,494$ & $-1,476$ & $-1,357$ \\
\hline Services & $-1,624$ & $-1,167$ & $-1,638$ & $-1,904$ & $-1,966$ & $-2,094$ & $-2,001$ & $-2,094$ \\
\hline Receipts & 522 & 650 & 615 & 627 & 656 & 798 & 852 & 911 \\
\hline Expenditure & $-2,146$ & $-1,817$ & $-2,253$ & $-2,531$ & $-2,622$ & $-2,892$ & $-2,853$ & $-3,005$ \\
\hline Of which: aid-related imports & -205 & -477 & -612 & -493 & -456 & -408 & -403 & -370 \\
\hline Income & $-1,321$ & -779 & $-1,015$ & $-1,074$ & $-1,197$ & -676 & -750 & -821 \\
\hline Receipts & 27 & 26 & 27 & 30 & 31 & 35 & 38 & 41 \\
\hline Expenditure & $-1,348$ & -805 & $-1,042$ & $-1,103$ & $-1,228$ & -711 & -788 & -862 \\
\hline Of which: interest payments ${ }^{1}$ & -493 & -555 & -70 & -23 & -27 & -225 & -262 & -291 \\
\hline Current transfers & 1,231 & 1,401 & 887 & 875 & 891 & 951 & 1,014 & 1,083 \\
\hline Of which: official aid & 1,019 & 1,277 & 805 & 781 & 783 & 840 & 893 & 952 \\
\hline Capital and financial account & 1,007 & 458 & 2,963 & 3,178 & 2,594 & 2,149 & 1,438 & 1,561 \\
\hline Capital account & 145 & 144 & 1,078 & 754 & 640 & 697 & 722 & 748 \\
\hline Official & 223 & 227 & 711 & 850 & 742 & 809 & 844 & 880 \\
\hline Private & -78 & -83 & -89 & -96 & -103 & -112 & -121 & -132 \\
\hline Capital transfers (HIPC/MDRI) & $\ldots$ & $\ldots$ & 8,541 & $\ldots$ & $\ldots$ & & & \\
\hline Of which: from IMF & $\ldots$ & $\ldots$ & 456 & $\ldots$ & $\ldots$ & & & \\
\hline Debt stock reduction (HIPC/MDRI) & $\ldots$ & $\cdots$ & $-8,086$ & $\ldots$ & $\cdots$ & & & \\
\hline Financial account & 862 & 314 & 1,885 & 2,424 & 1,955 & 1,452 & 716 & 813 \\
\hline Official capital & -455 & -315 & 724 & 1,273 & 1,024 & 475 & 820 & 587 \\
\hline Gross disbursements & 92 & 485 & 800 & 1,337 & 1,099 & 938 & 918 & 666 \\
\hline Scheduled amortization ${ }^{2}$ & -547 & -800 & -76 & -64 & -75 & -464 & -99 & -80 \\
\hline Private capital (net) & 1,317 & 629 & 1,162 & 1,151 & 931 & 977 & -104 & 227 \\
\hline Of which: Foreign direct investment & 1,713 & 629 & 805 & 1,130 & 1,051 & 854 & 888 & 984 \\
\hline Other private non-banking sector ${ }^{3}$ & -395 & 0 & 357 & 20 & -120 & 123 & -992 & -757 \\
\hline Balance before errors and omissions & -832 & -665 & 434 & 345 & 35 & 31 & 111 & 314 \\
\hline Errors and omissions & -115 & -247 & 0 & 0 & 0 & 0 & 0 & 0 \\
\hline Overall balance & -946 & -912 & 434 & 345 & 35 & 31 & 111 & 314 \\
\hline Financing & 768 & 469 & $-5,174$ & -449 & -136 & -154 & -238 & -427 \\
\hline Net change in non-Fund arrears ${ }^{4}$ & 781 & 907 & $-4,465$ & 0 & 0 & 0 & 0 & 0 \\
\hline Net banking sector reserves (increase $=-$ ) & -13 & -438 & -709 & -449 & -136 & -154 & -238 & -427 \\
\hline Central bank & -52 & 8 & -661 & -355 & -16 & -126 & -153 & -332 \\
\hline Of which: net Fund credit & -137 & 154 & -385 & 151 & 152 & 0 & 0 & -52 \\
\hline Commercial banks & 39 & -445 & -48 & -94 & -120 & -28 & -85 & -95 \\
\hline Financing need before exceptional assistance & -178 & -443 & $-4,740$ & -104 & -101 & -123 & -128 & -113 \\
\hline Exceptional financing & 178 & 443 & 4,542 & 0 & 0 & 0 & 0 & 0 \\
\hline Consolidation of arrears & 0 & 0 & 4,465 & 0 & 0 & 0 & 0 & 0 \\
\hline Debt relief ${ }^{5}$ & 178 & 443 & 78 & 0 & 0 & 0 & 0 & 0 \\
\hline \multirow[t]{2}{*}{ Residual financing need (overfinancing $=+$ ) } & 0 & 0 & -197.6 & -104 & -101 & -123 & -128 & -113 \\
\hline & \multicolumn{8}{|c|}{ (Percent of GDP, unless otherwise indicated) } \\
\hline Memorandum items: & & & & & & & & \\
\hline Debt service after debt relief (percent of exports of goods and services) & 14.1 & 21.2 & 2.9 & 1.0 & 1.2 & 1.3 & 1.3 & 1.6 \\
\hline Current account balance (including official transfers, but before debt relief) & -15.9 & -10.1 & -20.0 & -21.5 & -18.0 & -13.6 & -7.9 & -6.8 \\
\hline Current account balance (excluding official transfers and debt relief) & -24.6 & -21.6 & -26.4 & -27.4 & -23.5 & -19.0 & -13.2 & -12.0 \\
\hline Current account balance (including official transfers and debt relief) & -14.3 & -6.1 & -19.4 & -21.5 & -18.0 & -13.6 & -7.9 & -6.8 \\
\hline Current account balance (excluding official transfers, but including debt relief) & -23.1 & -17.6 & -25.7 & -27.4 & -23.5 & -19.0 & -13.2 & -12.0 \\
\hline Gross official reserves (in millions of U.S. dollars) & 78 & 999 & 1,257 & 1,359 & 1,649 & 1,927 & 2,080 & 2,360 \\
\hline Weeks of non-aid-related imports of goods and services & 0.8 & 7.2 & 7.5 & 7.9 & 8.6 & 10.1 & 10.1 & 11.1 \\
\hline
\end{tabular}

Sources: Congolese authorities; and IMF staff estimates and projections.

${ }^{1}$ Including interest due to the IMF.

${ }^{2}$ Excluding principal repayments to the IMF.

${ }^{3}$ Including unrecorded transactions. The latter may be substantial given weaknesses in statistics.

${ }^{4}$ Mainly arrears to Paris Club creditors.

${ }^{5}$ Including debt relief provided by the IMF and assumes HIPC Completion point by end June 2010. 
Table 5. The Democratic Republic of the Congo: Tentative Schedule of Disbursements and

Reviews Under the ECF Arrangement, 2010-12

\begin{tabular}{|c|c|c|}
\hline Date & Disbursement & Conditions \\
\hline June 30,2010 & SDR 49.493 million & $\begin{array}{l}\text { Completion of first review, based on } \\
\text { observance of performance criteria at end- } \\
\text { December } 2009 \text {. }\end{array}$ \\
\hline December 31, 2010 & SDR 49.493 million & $\begin{array}{l}\text { Completion of second review, based on } \\
\text { observance of performance criteria at end- } \\
\text { June } 2010 \text {. }\end{array}$ \\
\hline June 30, 2011 & SDR 49.493 million & $\begin{array}{l}\text { Completion of third review, based on } \\
\text { observance of performance criteria at end- } \\
\text { December } 2010 \text {. }\end{array}$ \\
\hline December 31, 2011 & SDR 49.493 million & $\begin{array}{l}\text { Completion of fourth review, based on } \\
\text { observance of performance criteria at end- } \\
\text { June } 2011 \text {. }\end{array}$ \\
\hline June 30, 2012 & SDR 49.493 million & $\begin{array}{l}\text { Completion of fifth review, based on } \\
\text { observance of performance criteria at end- } \\
\text { December } 2011 \text {. }\end{array}$ \\
\hline December 15, 2012 & SDR 49.492 million & $\begin{array}{l}\text { Completion of sixth review, based on } \\
\text { observance of performance criteria at end- } \\
\text { June } 2012 \text {. }\end{array}$ \\
\hline
\end{tabular}


Table 6. Democratic Republic of the Congo: Indicators of Capacity to Repay the Fund, 2010-22

\begin{tabular}{|c|c|c|c|c|c|c|c|c|c|c|c|c|c|}
\hline & 2010 & 2011 & 2012 & 2013 & 2014 & 2015 & 2016 & 2017 & 2018 & 2019 & 2020 & 2021 & 2022 \\
\hline \multicolumn{14}{|l|}{ Total obligations on existing and prospective credit ${ }^{1}$} \\
\hline (In millions of SDRs) & 37.5 & 0.4 & 1.3 & 1.5 & 1.5 & 33.9 & 62.7 & 82.3 & 96.9 & 83.4 & 55.0 & 35.1 & 15.2 \\
\hline Principal & 37.1 & 0.0 & 0.0 & 0.0 & 0.0 & 32.4 & 61.3 & 81.1 & 95.9 & 82.6 & 54.4 & 34.6 & 14.8 \\
\hline Charges and interest ${ }^{2}$ & 0.4 & 0.0 & 1.0 & 1.1 & 1.2 & 1.1 & 1.0 & 0.8 & 0.6 & 0.4 & 0.2 & 0.1 & 0.0 \\
\hline (In millions of U.S. dollars) & 57.5 & 0.5 & 2.0 & 2.3 & 2.3 & 52.1 & 96.1 & 126.2 & 148.7 & 127.9 & 84.4 & 53.8 & 23.4 \\
\hline In percent of exports of goods and services & 0.8 & 0.0 & 0.0 & 0.0 & 0.0 & 0.5 & 0.8 & 1.0 & 1.1 & 0.9 & 0.6 & 0.3 & 0.1 \\
\hline In percent of GDP & 0.5 & 0.0 & 0.0 & 0.0 & 0.0 & 0.3 & 0.5 & 0.6 & 0.6 & 0.5 & 0.3 & 0.2 & 0.1 \\
\hline In percent of quota & 7.0 & 0.1 & 0.2 & 0.3 & 0.3 & 6.4 & 11.8 & 15.4 & 18.2 & 15.6 & 10.3 & 6.6 & 2.9 \\
\hline In percent of gross international reserves & 0.0 & 0.0 & 0.0 & 0.0 & 0.0 & 0.0 & 0.0 & 0.0 & 0.0 & 0.0 & 0.0 & 0.0 & 0.0 \\
\hline \multicolumn{14}{|l|}{ Fund credit outstanding (end-period) } \\
\hline In millions of SDRs & 259.4 & 358.3 & 457.3 & 457.3 & 457.3 & 424.9 & 363.6 & 282.5 & 186.5 & 103.9 & 49.5 & 14.8 & 0.0 \\
\hline In millions of U.S. dollars & 397.7 & 548.0 & 700.0 & 700.8 & 701.4 & 651.8 & 557.8 & 433.4 & 286.2 & 159.5 & 75.9 & 22.8 & 0.0 \\
\hline In percent of exports of goods and services & 5.6 & 6.6 & 7.9 & 6.7 & 6.4 & 5.9 & 4.7 & 3.5 & 2.2 & 1.1 & 0.5 & 0.1 & 0.0 \\
\hline In percent of GDP & 3.1 & 4.0 & 4.8 & 4.4 & 4.0 & 3.5 & 2.8 & 2.0 & 1.2 & 0.6 & 0.3 & 0.1 & 0.0 \\
\hline In percent of quota & 95.7 & 67.2 & 85.8 & 85.8 & 85.8 & 79.7 & 68.2 & 53.0 & 35.0 & 19.5 & 9.3 & 2.8 & 0.0 \\
\hline In percent of total external debt & 3.1 & 3.4 & 3.8 & 3.6 & 3.4 & 3.2 & 2.8 & 2.3 & 1.7 & 1.1 & 0.5 & 0.2 & 0.0 \\
\hline In percent of gross international reserves & 32.1 & 41.5 & 44.2 & 37.5 & 34.3 & 32.5 & 27.2 & 19.9 & 12.4 & 0.1 & 0.0 & 0.0 & 0.0 \\
\hline \multicolumn{14}{|l|}{ Memorandum items } \\
\hline Exports of goods and services (in millions of U.S. dollars) & 7,097 & 8,322 & 8,782 & 9,529 & 10,171 & 10,880 & 12,029 & 12,753 & 13,440 & 14,191 & 14,977 & 15,791 & 16,633 \\
\hline Quota (in millions of SDRs) & 533 & 533 & 533 & 533 & 533 & 533 & 533 & 533 & 533 & 533 & 533 & 533 & 533 \\
\hline Gross international reserves (in millions of U.S. dollars) & 1,257 & 1,359 & 1,649 & 1,927 & 2,080 & 2,360 & 2,641 & 2,833 & 3,023 & 3,233 & 3,456 & 3,692 & 3,941 \\
\hline
\end{tabular}

Sources: IMF staff estimates and projections.

${ }^{1}$ Assumes hypothetical achievement of the completion point under the enhanced HIPC and debt relief under the MDRI in mid-2010.

${ }^{2}$ Upon the effectiveness of the ammendments to the PRGT instrument, there is a temporary interest waiver through end-2011 on ECF outstanding obligations and drawings. 


\title{
APPENDIX I \\ TRANSLATED FROM FRENCH
}

\section{DEMOCRATIC REPUblic OF THE CONGO \\ LETTER OF INTENT}

Kinshasa, June 15, 2010

\begin{abstract}
Mr. Dominique Strauss-Kahn
Managing Director

The International Monetary Fund

Washington, D.C. 20431, U.S.A
\end{abstract}

\section{Dear Mr. Managing Director}

1. The Democratic Republic of the Congo continues to advance its socioeconomic development agenda, which is being supported by the international community. There has been good progress in fostering national reconciliation, restoring peace in the eastern provinces, and stabilizing the economy following the adverse effects of the global financial crisis. The Government also renegotiated the Sino-Congolese Cooperation Agreement (SCCA) involving large mining and investment projects to make it consistent with debt sustainability. Nevertheless, daunting challenges remain, including national calamities, persisting security issues in the northern and eastern provinces, and the implementation of the constitutionally mandated fiscal decentralization involving the provinces.

2. Despite these challenges, the Democratic Republic of the Congo implemented satisfactorily its economic program supported by the IMF's Extended Credit Facility (ECF). All quantitative performance criteria at end-December 2009 were observed, and almost all of the structural benchmarks were implemented, albeit with some delay. These policies and reforms helped stabilize the exchange rate, reduce inflation, and nurture economic growth.

3. The 2010 economic program aims to address the economic challenges facing the country consistent with the priorities set forth in our Poverty Reduction and Growth Strategy (PRGS). It focuses on enhancing macroeconomic stability, increasing investment in physical and human capital, and implementing structural reforms to strengthen public financial management, enhance central bank independence, and boost our economy's supply response. The policies and reforms to achieve the program's objectives are set out in the attached Memorandum of Economic and Financial Policies (MEFP). In this context, the government of Democratic Republic of the Congo requests the completion of the first review of the ECF arrangement.

4. We believe that the policies and reforms set forth in the attached MEFP are adequate to achieve our program's objectives. Nevertheless, we are committed to take any further measures that may prove necessary for achieving the program's objectives. During the 
period covered by the three-year arrangement, the Democratic Republic of the Congo will consult with the staff of the IMF on the adoption of any additional measures deemed to be appropriate.

5. The Democratic Republic of the Congo will provide the IMF with any information it may request for monitoring the implementation of the program's economic and financial policies. Furthermore, the government will conduct with the IMF a subsequent review of the first annual program (covering the period January 2010-June 2010) no later than end-December 2010 and an initial review of the second year of the program (July 2010-June 2011) no later than endJune 2011. It intends to make the contents of this letter, the attached MEFP, the Technical Memorandum of Understanding (TMU), and the related IMF staff report available to the public. Therefore, it authorizes the Fund to publish these documents on its website once the Executive Board concludes the first review of the ECF arrangement.

Sincerely yours $/ \mathrm{s} /$

Adolphe Muzito

Prime Minister

Attachments: Memorandum of Economic and Financial Policies Technical Memorandum of Understanding 


\section{APPENDIX I \\ ATtAChMENT I \\ TRANSLATED FROM FRENCH \\ Democratic RePublic OF THE CONGo \\ Memorandum of ECONOMiC ANd FinanCial Policies, 2009-12}

Kinshasa, June 15, 2010

\section{INTRODUCTION}

1. This memorandum describes the economic and financial policies as well as the structural reforms that the Government intends to implement in its program supported by an arrangement under the Extended Credit Facility (ECF) of the International Monetary Fund (IMF) for the remaining period July 2010 to June 2012. Through this program, the Government is pursuing efforts to reestablish the conditions for lasting political, economic, and social stability. In particular, the proposed policies and reforms aim to address the legacy of decades of poor economic management, armed conflicts, corruption, and disregard for the rule of law.

\section{RECENT ECONOMIC DEVELOPMENTS}

2. The global financial crisis weakened macroeconomic performance. Real GDP growth is estimated to have declined from 6.2 percent in 2008 to 2.8 percent in 2009 owing to weak mining and tertiary sector activities. Year-on-year inflation rose from 27.6 percent at end-2008, to 53.4 percent by end-2009 in response to a weakening of the Congolese franc against the U.S. dollar as exports declined sharply. However, annualized inflation declined to 15 percent at end-April owing to a tightening of monetary policy in late 2009 and a supportive fiscal policy. The external current account deficit narrowed by 6 percentage points of GDP, reflecting largely lower profit repatriation because of weak mining activity and higher official transfers. The special and general SDR allocations together with external budget support boosted international reserves to US\$1 billion at end-2009, up from US\$78 million at end-2008.

3. Despite unfavorable economic conditions, the Government maintained macroeconomic discipline. It limited the 2009 domestic fiscal deficit to 2.5 percent of GDP compared to a programmed deficit of 3.1 percent of GDP. As a result, the Government reduced its net credit from the banking system despite delays in the disbursement of foreign assistance (equivalent to 0.8 percent of GDP) and of the second tranche of the SCCA signing bonus (1.1 percent of GDP). Fiscal performance remained strong through the second quarter of 2010. The recovery in the mining sector and continued improvements in tax administration boosted revenue - mainly income tax and nontax revenues - by 0.7 percentage points of GDP above program projection. Two-thirds of the revenue overage was used to finance additional security-related spending and the front loading of capital spending for infrastructure in the context of the preparation of the $50^{\text {th }}$ anniversary of the country's independence. The other third was used to reduce net credit to government from the banking system. 
4. Prudent monetary policy helped reduce inflation. The Central Bank of Congo (BCC) stepped up sales of central bank bills and raised its policy rate to 70 percent during the second half of 2009. However, the lack of coordination between the BCC and the Treasury in liquidity management resulted in marginally higher-than-programmed base money growth at end-year (24.9 percent instead of programmed 19.6 percent). Nevertheless, continued prudent monetary policy supported by improved fiscal policy contributed to a significant disinflation during the first four months of 2010. In view of this, the BCC reduced its policy rate in three steps to 42 percent between March and May 2010.

5. Program performance was satisfactory. All quantitative performance criteria and indicative targets through end-December 2009 were observed, except for the indicative targets on base money and wage arrears (MEFP, Table I.1). ${ }^{1}$ Moreover, although three out of ten quantitative indicative targets - net foreign assets, net domestic assets, and base money - were not observed at end-March 2010, the deviations were relatively small and corrective measures have been taken to ensure that the program remains on track. In particular, in April the BCC increased its purchases of foreign exchange from commercial banks and sales of central bank bills. On the structural front, we observed almost all of the benchmarks, albeit with some delay. The restructuring of a large commercial bank with systemic importance is requiring more time than originally envisaged, and the central bank, in consultation with the IMF's Monetary and Capital Markets department, launched the restructuring process in May 2010, which is expected to be completed before the end of the year. Regarding the benchmark on government submission to parliament of an organic budget law, upon judicial review it was determined that an "organic" law is inconsistent with the Constitution, including the provision related to the prohibition of borrowing by provinces. As a result, in March 2010 the Government submitted to parliament a budget finance law, consistent with the Constitution. The continuous structural benchmark regarding the timely production of fiscal accounts without foreign financing spending was observed beginning in June 2010 because of the impact of the December 2009 civil service strike that hampered the proper functioning of the expenditure management chain. However, the continuous structural benchmark regarding the timely production of fiscal accounts with foreign financing spending was not observed because of difficulties in obtaining information on several foreign financed projects.

\footnotetext{
${ }^{1}$ The accumulation of wage arrears vis-à-vis diplomats based overseas during the last quarter of the year amounted to CGF 2 billion or 0.4 percent of the total wage bill. This was because wage payments to diplomats are done outside the automated system in place for all other civil servants.
} 


\section{EConomic ANd Financial Policies for the Remainder of 2010}

\section{A. Macroeconomic framework}

6. The Democratic Republic of the Congo's economic objectives remain broadly unchanged from those set out in the November 2009 MEFP. The firming up of international commodity prices and increased investment (including that under the SCCA) are expected to support real GDP growth of 5.4 percent in 2010. Year-on-year inflation is targeted to fall to 15 percent supported by prudent macroeconomic policies. The external current account deficit is projected to widen mainly because of higher investment-related imports financed largely by development assistance and foreign direct investment. Gross international reserves are targeted to reach US $\$ 1.2$ billion (or the equivalent of 7.5 weeks of non-aid imports).

\section{B. Macroeconomic policies}

7. The government's fiscal policy remains focused on curtailing government recourse to central bank financing. The domestic fiscal balance is targeted at 1.0 percent of GDP ( 0.3 percentage points lower than originally programmed), implying 1.5 percentage point of GDP reduction in net credit to the government. ${ }^{2}$

8. Revenue mobilization remains a key pillar of the program. The objective is to raise the ratio of domestic revenue (excluding the signing bonus under the SCCA) from 15.8 percent of GDP in 2009 to 18.1 percent in 2010 (1.4 percentage points of GDP higher than programmed). This will be facilitated by the stronger than programmed revenue performance realized during the first quarter of 2010, as well as by new policy measures. Regarding the latter, the Government: (i) is aligning the reference fuel prices for taxation purposes with world market prices ( 0.3 percentage points of GDP); (ii) is enforcing the payment of personal income taxes by political institutions ( 0.2 percentage points of GDP); and (iii) eliminated ad hoc income tax and tariff exemptions ( 0.2 percentage points of GDP). The Government's policy is to save all revenue beyond that projected under the program, including the second tranche of the SCCA signing bonus, and we will consult with IMF staff before considering the use of such revenues.

9. The Government will implement a prudent expenditure policy while creating the fiscal space for priority programs. It will limit domestically financed spending to 19.1 percent of GDP, about 1 percentage point of GDP higher than in the original program. While the wage bill and expenditure on goods and services will remain broadly unchanged, we have decided to reduce domestically financed investment (by 0.6 percentage points of GDP) to make room for higher spending on the preparation for 2011 presidential elections and national security. We believe, however, that the impact of lower domestically financed government investment will be minimal given the low project implementation rate and the large investment program under the SCCA

\footnotetext{
${ }^{2}$ In order to compare original program projection figures (in percent of GDP) with the outturn, the original program projection figures were rebased using the new GDP estimate for 2010.
} 
(equivalent to 5.6 percent of GDP). The revised program for 2010 also provides for: (i) a largerthan-envisaged central bank deficit on account of the higher than expected cost of monetary policy ( 0.3 percent of GDP); (ii) the repayment of government obligations to a systemically important commercial bank that is being restructured ( 0.2 percent of GDP); and (iii) the repayment of arrears in transfers to the provinces and the tax collection agencies $(0.4$ percent of GDP). Ongoing reforms to bolster expenditure management will help ensure strict adherence to the revised expenditure policy.

10. To ensure the long-term sustainability of its external debt, the central government, local governments and the BCC are committed to a prudent external debt management policy. It will rely on grants and highly concessional loans with a grant element exceeding 35 percent for financing projects with the highest social and economic rates of return. The government will refrain from contracting or guaranteeing new debt that would jeopardize debt sustainability and will consult with Fund staff prior to making any such commitments or guarantees.

11. The primary objective of monetary policy in 2010 is to reduce further inflation. The growth of base money is targeted at 31 percent in 2010, in line with the expansion of nominal GDP. The BCC will maintain a prudent monetary policy and a positive real policy interest rate. It will continue to rely on the sale of central bank bills to manage liquidity. Further, it will limit intervention in the foreign exchange market to smoothing short-term exchange rate volatility in line with the floating exchange rate regime and the BCC's foreign reserve target.

\section{Structural reforms}

12. Structural reforms will continue to focus on modernizing tax and customs administration, strengthening public financial management (PFM), restructuring and recapitalizing the $\mathrm{BCC}$, and enhancing the economy's supply response. The detailed and updated measures and their timetable are provided in the attached Matrix of Economic and Financial Policy Measures.

13. Modernizing tax and customs administration and PFM remains a priority in 2010. On tax administration, key reforms envisaged include the adoption of a value added tax (VAT) law, expansion of one-stop customs widows, and establishing new tax centers in key provinces for medium-sized enterprises in anticipation of the introduction of the VAT in 2012. On PFM, the modernization of the expenditure management system will be guaranteed by the adoption by end-December 2010 of a new finance law, which will trigger a series of reforms, at both the central and provincial levels, that will help streamline budget planning, execution and monitoring. The law will also define the devolution of functions, and their modalities, from the central government to provinces while taking into account the PFM capacities of provinces. The law will also define the modalities of transfer to provinces of national resources. It also envisages strict borrowing limits to help avoid loss of fiscal control and excessive borrowing. With the adoption and implementation of a new procurement code, the Government aims at improving transparency of public spending. 
14. Financial sector reforms will focus on reinforcing the BCC's role and independence in implementing monetary policy and strengthening banking supervision. Given that the first steps of reorganizing/restructuring of the $\mathrm{BCC}$ have been achieved (i.e., the appointment of all general directors and the establishment of new organic units in line with the organizational structure developed with technical assistance from the IMF's MCM department), the focus will be on privatizing the Mint and the BCC hospital and rationalizing the BCC's pension system. Progress on these fronts will help reduce the operational costs of the BCC. Further, the groundwork for recapitalizing the $\mathrm{BCC}$ is underway and we expect to submit the recapitalization law to Parliament by end-September 2010. On the other front, the focus will be on restructuring weak commercial banks, which is essential for enhancing financial intermediation, and strengthening banking supervision. With the completion of central bank audits of nine key banks, the BCC will develop restructuring plans for those considered most fragile with regard to their prudential ratios.

15. Reforms to improve the economy's supply response are critical for strengthening economic growth and reducing poverty. The Government is finalizing its work on advancing the ongoing reform of public enterprises, especially those that provide growth-critical services. In this context, we are also in the process developing plans to minimize the social cost of the restructuring, of enterprises, especially on public enterprise employees. The Government is also improving governance and the business climate through enhancing property rights protection by harmonizing domestic regulations with those under the OHADA protocol and adhering to the Extractive Industries Transparency Initiative.

\section{Program monitoring and related issues}

16. The monitoring of the program will continue to be carried out based on semiannual reviews of quantitative performance criteria and quarterly indicative targets while structural reforms will be assessed in the context of semiannual reviews (Tables 1 and 2). The revised program establishes performance criteria for end-December 2010 and indicative targets for endSeptember 2010. The end-June 2010 quantitative performance criteria and benchmarks remain unchanged from those established in the November 2009 MEFP. The second review is expected to be completed by end-December 2010 and the third review by end-June 2011. The attached TMU provides the definitions and adjustors relevant for the quantitative performance criteria and benchmarks from July 1, 2010 onwards. The TMU accompanying the November 2009 MEFP remains applicable to the quantitative performance criteria and indicative targets through endJune 2010. A safeguards updates assessment was completed in April 2010, as required under the IMF's safeguards policy. The authorities provided to staff an audit report of selected performance criteria. In addition, the BCC drafted an action plan to implement International Financial Regulation Standards, which is expected to be adopted in December 2010.

17. The Government will reinforce its monitoring mechanism through extensive interdepartment consultation and exchange of information, under the supervision of the Prime Minister's Office, as outlined in its November 2009 MEFP. 
18. The Government has recently completed an update on the implementation its first PRGS. It is also in process of preparing a second generation PRGS. It launched the process in March 2010 and the work is expected to be completed by end-2010.

Sincerely yours,

/s/

Mapon Matata Ponyo

Minister of Finance $/ \mathrm{s} /$ $/ \mathrm{s} /$

Jean-Baptiste Ntwawa Minister of Budget
Jean-Claude Masangu Mulongo BCC Governor 
Table 1.1. Democratic Republic of the Congo: Quarterly Quantitative Performance Criteria and Indicative Targets, 2009 - 2010

\begin{tabular}{|c|c|c|c|c|c|c|c|c|c|c|c|c|c|c|c|c|c|c|}
\hline & \multirow{2}{*}{$\frac{2008}{\frac{\text { Stock Dec. }}{\text { Prel }}}$} & \multicolumn{5}{|c|}{2009 Cumulative change ${ }^{2}$} & \multirow{2}{*}{$\frac{2009}{\frac{\text { Stock Dec. }}{\text { Act. }}}$} & \multirow[b]{2}{*}{$\overline{\text { Indicative }}$} & \multirow[b]{2}{*}{$\begin{array}{c}\text { Indicative } \\
\text { Adj. }\end{array}$} & \multicolumn{4}{|c|}{2010 Cumulative change $\mathrm{e}^{3.4}$} & \multirow[b]{2}{*}{$\frac{S \text { Se }}{\text { Indicative }}$} & \multirow[b]{2}{*}{ Rept. } & \multirow{2}{*}{\multicolumn{2}{|c|}{$\begin{array}{l}\text { Dec. } \\
\text { Indicative Perf. Criteria }\end{array}$}} & \multirow{2}{*}{$\frac{2010}{\frac{\text { Stock Dec }}{\text { Prog }}}$} \\
\hline Stoc & & Perf. Criteria & $\begin{array}{l}\text { Perf. Criteria } \\
\text { Adj. }\end{array}$ & $\frac{\text { Dec. }}{\text { Act }}$ & Difference & Obesrvation & & & & $\begin{array}{l}\text { March } \\
\text { Act. }\end{array}$ & Difiference & Obesrvation & $\frac{\text { June }}{\overline{\text { Perf. Criteria }}}$ & & & & & \\
\hline \multicolumn{19}{|l|}{ Performance criteria } \\
\hline $\begin{array}{l}\text { Floor on net foreign assets of the } \mathrm{BCC}^{5} \\
\text { (U.S.S. millions) }\end{array}$ & -610 & 35 & -109 & -3 & 106 & Observed & -613 & -23 & 14 & 15 & 1 & Observed & 144 & 510 & 648 & 576 & 613 & 0 \\
\hline Ceiling on net domestic assets of the $\mathrm{BCC}^{5}$ & 773,772 & 51,343 & 155,070 & 95,545 & $-59,525$ & Observed & 869,089 & 42,225 & 7,974 & $-1,748$ & $-9,722$ & Observed & $-69,580$ & $-180,079$ & $-266,056$ & $-825,115$ & $-869,089$ & 0 \\
\hline Celiling on net bank credit to government & 262,875 & $-105,837$ & $-2,110$ & $-80,522$ & $-78,412$ & Observed & 182,353 & $-80,725$ & $-114,976$ & $-108,355$ & 6,622 & Not Observed & $-122,644$ & -99,636 & $-183,140$ & $-154,723$ & $-182,353$ & 0 \\
\hline $\begin{array}{l}\text { Ceiling on the contracting or guaranteeing of } \\
\text { new nonconcessionale lexternal debt with } \\
\text { original maturity of more than one year } \\
\text { by the government, including the EADs or } \\
\text { the BCC }\end{array}$ & & 0 & 0 & 0 & 0 & Observed & & 0 & & 0 & & $\ldots$ & 0 & 0 & 0 & 0 & 0 & 0 \\
\hline 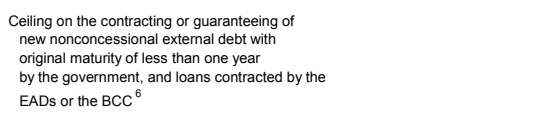 & & 0 & & 0 & 0 & Observed & & 0 & & $\ldots$ & & & 0 & 0 & 0 & 0 & 0 & 0 \\
\hline 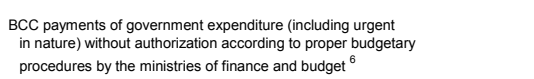 & & 0 & & 0 & 0 & Observed & & 0 & & $\ldots$ & & $\ldots$ & 0 & 0 & 0 & 0 & 0 & 0 \\
\hline Accumulation of External payments arrears ${ }^{6}$ & & 0 & & 0 & 0 & Observed & & 0 & & 0 & & $\ldots$ & 0 & 0 & 0 & 0 & 0 & 0 \\
\hline \multicolumn{19}{|l|}{ Indicative targets } \\
\hline Narrow base money & 378,098 & 75,016 & 75,016 & 93,624 & 18,608 & Not Observed & 471,722 & 26,001 & 26,001 & 7,393 & $-18,608$ & Observed & 22,834 & 145,089 & 148,586 & $-453,114$ & $-471,722$ & 0 \\
\hline Domestic balance (cumulative from the beginning of the year) & & $-285,251$ & & $-226,761$ & 58,490 & Observed & & 55,623 & & 78,129 & 22,506 & Observed & 79,117 & $-57,794$ & $-3,776$ & $-153,960$ & $-116,768$ & \\
\hline Accumulation of wage arrears & & 0 & & 2,000 & 2,000 & Not Observed & & 0 & & $\ldots$ & & $\ldots$ & 0 & 0 & 0 & 0 & 0 & \\
\hline 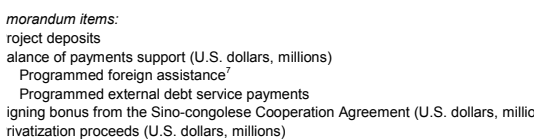 & Ilions) & $\begin{array}{l}0 \\
174 \\
359 \\
185 \\
250 \\
45\end{array}$ & $\begin{array}{l}0 \\
\ldots \\
\ldots \\
\ldots \\
\ldots\end{array}$ & $\begin{array}{r}-4,460 \\
122 \\
187 \\
65 \\
125 \\
72\end{array}$ & $\begin{array}{r}-4,460 \\
-52 \\
-13 \\
-120 \\
-125 \\
27\end{array}$ & $\begin{array}{l}\cdots \\
\cdots \\
\cdots\end{array}$ & $\begin{array}{c}9,516 \\
\cdots \\
\cdots \\
\cdots \\
\cdots \\
\cdots\end{array}$ & $\begin{array}{r}11,661 \\
-37 \\
0 \\
37 \\
0 \\
0\end{array}$ & $\begin{array}{r}11,661 \\
\ldots \\
\ldots \\
\ldots \\
\ldots\end{array}$ & $\begin{array}{r}-13,976 \\
0 \\
12 \\
12 \\
0 \\
0\end{array}$ & $\begin{array}{r}-25,637 \\
37 \\
12 \\
-25 \\
0 \\
0\end{array}$ & $\begin{array}{l}\ldots \\
\cdots \\
\cdots \\
\ldots\end{array}$ & $\begin{array}{r}9,516 \\
378 \\
451 \\
74 \\
0 \\
0\end{array}$ & $\begin{array}{r}9,516 \\
341 \\
451 \\
110 \\
0 \\
0\end{array}$ & $\begin{array}{r}9,516 \\
568 \\
678 \\
110 \\
0 \\
0\end{array}$ & $\begin{array}{r}0 \\
583 \\
730 \\
147 \\
0 \\
0\end{array}$ & $\begin{array}{r}0 \\
647 \\
793 \\
146 \\
125 \\
0\end{array}$ & $\begin{array}{r}11,661 \\
\cdots . \\
\cdots . \\
\cdots . \\
\ldots . .\end{array}$ \\
\hline
\end{tabular}

Source: Congolese authorities and IMF staff estimates and projections.

${ }_{2}^{1}$ For definition and adjustors see the attached Program Monitoring Section of Memorandum of Economic and Financial Polices and the Technical Memorandum of Understanding.

${ }_{3}^{2}$ Cumulative changes are calculated from end-December 2008

${ }^{4}$ The performance criteria and indicative targets for end-June and March 2010 are those estabished in IMF Country Report No. 10/88.

${ }^{5}$ The stocks of net foreign assets and net domestic assets of the BCC are valued at the program exchange rates (USS1 = CGF 639.32; and 1 Euro = 905.07)

${ }^{6}$ These performance crititia will be monitored on a continuous basis.
${ }^{2}$ Non U.S. dollar denominated balance of payment support is valued at program exchange rates. 
Table I.2a. The Democratic Republic of the Congo: Structural Benchmarks, 2009-10

\begin{tabular}{|c|c|c|}
\hline Measure & Test dates & Status \\
\hline Restructuring one large commercial bank ${ }^{1 /}$ & End-Dec. 2009 & Observed with a delay; end-May 2010. \\
\hline $\begin{array}{l}\text { Adoption by the Council of Ministers of an action plan } \\
\text { prepared by the committee responsible for assessing tax } \\
\text { incentives and exemptions granted under the mining code, } \\
\text { investment code, and special convention. }\end{array}$ & End-Dec. 2009 & Observed with a delay; March 2010. \\
\hline $\begin{array}{l}\text { Implementation of ASYCUDA++ in the customs offices } \\
\text { in Kinshasa }\end{array}$ & $\begin{array}{l}\text { End- Dec. } \\
2009\end{array}$ & Observed. \\
\hline $\begin{array}{l}\text { Repayment plan for } 2008 \text { arrears based the internal audit } \\
\text { of the Inspection General des Finances and establish a } \\
\text { plan to identify and audit of any remaining government } \\
\text { arrears. }\end{array}$ & $\begin{array}{l}\text { End- Feb. } \\
2010\end{array}$ & Observed with a delay; March 2010. \\
\hline $\begin{array}{l}\text { Submission to Parliament of a draft organic law on public } \\
\text { finance that inter alia prohibits provinces from borrowing } \\
\text { from commercial banks and the Central Bank. }\end{array}$ & $\begin{array}{l}\text { End-March } \\
2010\end{array}$ & $\begin{array}{l}\text { Observed; Budget Finance Law without restrictions } \\
\text { on provincial borrowing was submitted to } \\
\text { parliament, in line with the constitution. }\end{array}$ \\
\hline $\begin{array}{l}\text { Generation and publishing of a monthly budget execution } \\
\text { tables (reconciled with the TOFE, the BCC, and the } \\
\text { monetary statistics), no later than } 30 \text { days after the end of } \\
\text { each month. }\end{array}$ & Continuous & Observed with a delay; starting from June 1, 2010. \\
\hline $\begin{array}{l}\text { Inclusion of externally financed expenditure in the } \\
\text { monthly budget execution tables (reconciled with the } \\
\text { TOFE, the BCC, and the monetary statistics), no later than } \\
3 \text { months after the end of each month. }\end{array}$ & Continuous & Not observed \\
\hline $\begin{array}{l}\text { Publication of mining sector partnership contracts } \\
\text { between public and private enterprises with } 60 \text { days of } \\
\text { signature (including information on signing bonuses, } \\
\text { taxation system, private shareholders, and members of the } \\
\text { board of directors). }\end{array}$ & Continuous & Observed. \\
\hline $\begin{array}{l}\text { No payment by the BCC of expenditures on behalf of the } \\
\text { government (including urgent expenditure payments) } \\
\text { without prior authorization by the Minister of Finance in } \\
\text { accordance with existing legislation. }\end{array}$ & Continuous & Observed. \\
\hline
\end{tabular}

Source: Congolese authorities; and IMF staff assessments.

${ }^{1 /}$ IMF staff and authorities agreed to postpone implementation of this measure, while awaiting the recommendations of the January/February 2010 MCM mission. 


\section{Table I.2b.The Democratic Republic of the Congo: Structural Conditionality and Macroeconomic Relevance, July-December 2010}

\begin{tabular}{|c|c|c|}
\hline Measure & Test date & Macroeconomic Rational \\
\hline $\begin{array}{l}\text { Adoption by the Parliament and promulgation of the law } \\
\text { introducing a modern single-rate VAT. }\end{array}$ & $\begin{array}{l}\text { End-December } \\
2010\end{array}$ & $\begin{array}{l}\text { Enhance revenue mobilization to achieve the fiscal } \\
\text { objectives of the program. }\end{array}$ \\
\hline $\begin{array}{l}\text { Establishment of the Kasumbalesa one-stop customs } \\
\text { window. }\end{array}$ & $\begin{array}{l}\text { End-July } \\
20101\end{array}$ & $\begin{array}{l}\text { Enhance revenue mobilization to achieve the fiscal } \\
\text { objectives of the program while reducing cost of } \\
\text { doing business. }\end{array}$ \\
\hline $\begin{array}{l}\text { Increase the border fuel price for taxation purposes } \\
\text { (PMFF) to at least one-third of the commercial price } \\
\text { (PMFC) of fuel imports starting from June 2010, the } \\
\text { authorities will gradually reduce the gap in order to reach } \\
\text { the target by end-November } 2010 \text {. }\end{array}$ & $\begin{array}{l}\text { End- } \\
\text { November } \\
2010\end{array}$ & $\begin{array}{l}\text { Reduce distortions in relative prices and limit } \\
\text { revenue losses. }\end{array}$ \\
\hline $\begin{array}{l}\text { Adoption by the Parliament and promulgation of the law } \\
\text { on government finance. }\end{array}$ & $\begin{array}{l}\text { End-December } \\
2010\end{array}$ & Develop a rule-based public financial management. \\
\hline $\begin{array}{l}\text { Submission to Parliament of the draft law on the } \\
\text { recapitalization of the BCC. }\end{array}$ & $\begin{array}{l}\text { End-October } \\
2010\end{array}$ & Enhance the independence of the central bank. \\
\hline $\begin{array}{l}\text { Generation and publishing of a monthly budget execution } \\
\text { tables (reconciled with the TOFE, the BCC, and the } \\
\text { monetary statistics), no later than } 30 \text { days after the end of } \\
\text { each month. }\end{array}$ & Continuous & Strengthen the monitoring of the program. \\
\hline $\begin{array}{l}\text { Inclusion of externally financed expenditure (excluding } \\
\text { exceptional spending) in the monthly budget execution } \\
\text { tables (reconciled with the TOFE, the BCC, and the } \\
\text { monetary statistics), no later than } 3 \text { months after the end } \\
\text { of each month. }\end{array}$ & Continuous & Strengthen the monitoring of the program. \\
\hline $\begin{array}{l}\text { Publication of mining sector partnership contracts } \\
\text { between public and private enterprises within } 60 \text { days of } \\
\text { signature (including information on signing bonuses, } \\
\text { taxation system, private shareholders, and members of the } \\
\text { board of directors). }\end{array}$ & Continuous & $\begin{array}{l}\text { Enhance economic governance and ensure } \\
\text { accountability for public resources management. }\end{array}$ \\
\hline $\begin{array}{l}\text { No payment by the } \mathrm{BCC} \text { of expenditures on behalf of the } \\
\text { government (including urgent expenditure payments) } \\
\text { without prior authorization by the Minister of Finance in } \\
\text { accordance with existing legislation. }\end{array}$ & Continuous & $\begin{array}{l}\text { Improve economic governance and public financial } \\
\text { management. }\end{array}$ \\
\hline
\end{tabular}

Source: Congolese authorities; and IMF staff assessments. 
Democratic Republic OF THE CONGO

Matrix of Economic and Financial Policy Measures, 2009 to 2011

\begin{tabular}{|c|c|c|c|}
\hline Sector & & Measures & Timetable \\
\hline \multicolumn{4}{|c|}{ I. Tax and budgetary policies and reforms } \\
\hline \multirow[t]{6}{*}{ A. Tax policy } & & Objective: Modernize the tax system & \\
\hline & 1. & $\begin{array}{l}\text { Reduce the number of low-yielding taxes: } \\
\text { - Adoption by the Council of Ministers of the report on } \\
\text { identifying low-yielding taxes. } \\
\text { - Submit to the Parliament draft laws on the elimination of } \\
\text { these taxes. }\end{array}$ & $\begin{array}{l}\text { Done. } \\
\text { End-June } 2010 .\end{array}$ \\
\hline & 2. & $\begin{array}{l}\text { Limit tax and customs incentives and exemptions: } \\
\text { - Establish a commission to evaluate the tax incentives and } \\
\text { exemptions in the mining. } \\
\text { Adoption by the Council of Ministers of the action plan, } \\
\text { which is elaborated by the Commission in charge of } \\
\text { evaluation of incentives and fiscal exemptions, granted in } \\
\text { the framework of the Mining Code, Investment Code and } \\
\text { special conventions. (structural benchmark) } \\
\text { Refrain from granting or renewing discretionary tax } \\
\text { incentives and exemptions outside the investment. }\end{array}$ & $\begin{array}{l}\text { Done. } \\
\text { Done. }\end{array}$ \\
\hline & 3. & $\begin{array}{l}\text { Reduce the number of nuisance charges and border levies: } \\
\text { - Produce a report identifying para fiscal taxes and border } \\
\text { fees, without a quid pro quo, and adopt an action plan to } \\
\text { eliminate them. } \\
\text { - Implement the action plan. }\end{array}$ & $\begin{array}{l}\text { End-December } \\
2009 ; \text { delayed to } \\
\text { end-July } 2010 . \\
\text { End-December } \\
2010 .\end{array}$ \\
\hline & 4. & $\begin{array}{l}\text { Implement a VAT: } \\
\text { - Adopt and enact the draft law establishing the modern } \\
\text { Single-rate VAT (Structural benchmark). } \\
\text { Collect the first VAT tax returns from businesses by the } \\
\text { DGI. }\end{array}$ & $\begin{array}{l}\text { End-December } 2010 \\
\text { End-January } 2012\end{array}$ \\
\hline & 5. & $\begin{array}{l}\text { Implement the new Customs Code: } \\
\text { - Adoption by the Council of Ministers. } \\
\text { - Submission to the Parliament. } \\
\text { - Promulgation by the President. }\end{array}$ & $\begin{array}{l}\text { Done. } \\
\text { Done. } \\
\text { End-December } \\
2010 . \\
\end{array}$ \\
\hline \multirow{2}{*}{$\begin{array}{l}\text { B. Customs } \\
\text { administration }\end{array}$} & & Objective: Modernize customs administration & \\
\hline & 1. & $\begin{array}{l}\text { Transfer customs activities from the Congolese Office of Control } \\
\text { (OCC) to General Directorate of Import and Excise Duties (DGDA): } \\
\text { - Adoption by the Council of Ministers of the new statutes of the } \\
\text { OCC and DGDA. } \\
\text { Modify the decree which determines the number of services } \\
\text { operating at borders. }\end{array}$ & $\begin{array}{l}\text { Done. } \\
\text { End-December } \\
2010 .\end{array}$ \\
\hline
\end{tabular}




\begin{tabular}{|c|c|c|c|}
\hline Sector & \multicolumn{2}{|r|}{ Measures } & \multirow{2}{*}{$\begin{array}{l}\text { Timetable } \\
\text { Done. }\end{array}$} \\
\hline & 2. & $\begin{array}{l}\text { Issuance by the Prime Minister of a decree to convert DGDA } \\
\text { into a Directorate-General of Customs reporting to the } \\
\text { Ministry of Finance. }\end{array}$ & \\
\hline & 3. & $\begin{array}{l}\text { Strengthen the rules requiring pre-inspection of imports by } \\
\text { BIVAC; for goods not yet subject to pre-inspection, DGDA } \\
\text { will determine their value by using the BIVAC database. }\end{array}$ & Done. \\
\hline & 4. & $\begin{array}{l}\text { Implement ASYCUDA++ in all customs offices where the } \\
\text { volume and type of trade justify it: } \\
\text { - At Kinshasa. (structural benchmark) } \\
\text { - At other customs office that account for either a minimum } \\
\text { of } 5 \text { percent of imports (CIF value) or a minimum of } \\
10 \text { percent of transit (CIF value). }\end{array}$ & $\begin{array}{l}\text { Done. } \\
\text { End-December } \\
2011 .\end{array}$ \\
\hline & 5. & $\begin{array}{l}\text { Introduce the one-stop window at all customs offices: } \\
\text { - Set up a one-stop window at Kasumbalesa (structural } \\
\text { benchmark). } \\
\text { - Set up a one-stop window at all other customs offices. }\end{array}$ & $\begin{array}{l}\text { End-July } 2010 \\
\text { End-December } \\
2011 .\end{array}$ \\
\hline \multirow{6}{*}{$\begin{array}{l}\text { C. Tax } \\
\text { administration }\end{array}$} & & Objective: Strengthen tax administration & \\
\hline & 1. & $\begin{array}{l}\text { Merge the DGRAD with the DGI: } \\
\text { - Set up an independent commission to study the } \\
\text { advisability and feasibility of merging the DGRAD with } \\
\text { the DGI. } \\
\text { - Adopt the report on the advisability and feasibility of } \\
\text { merging the DGRAD with the DGI. }\end{array}$ & $\begin{array}{l}\text { Done. } \\
\text { End-September } \\
2010 .\end{array}$ \\
\hline & 2. & $\begin{array}{l}\text { Extend the use of the new single tax identification number } \\
\text { (NIF) to all provinces. }\end{array}$ & $\begin{array}{l}\text { End-December } \\
2010 .\end{array}$ \\
\hline & 3. & $\begin{array}{l}\text { Transfer all enterprises that meet the eligibility criteria to the } \\
\text { DGE portfolio. }\end{array}$ & Done. \\
\hline & 4. & $\begin{array}{l}\text { Establish tax centers (CDIs) in all provinces to manage } \\
\text { medium-sized enterprises: } \\
\text { - Establish three CDIs: Bas Congo, Nord Kivu and Sud } \\
\quad \text { Kivu. } \\
\text { - Establish CDIs in all other provinces. }\end{array}$ & $\begin{array}{l}\text { End-December } 2010 \\
\text { (ongoing). } \\
\text { End-December } \\
2011 \text {. }\end{array}$ \\
\hline & 5. & $\begin{array}{l}\text { Finalize a study on the feasibility of implementing a } \\
\text { computerized revenue system. }\end{array}$ & End -March 2011. \\
\hline $\begin{array}{l}\text { D. Collection of } \\
\text { mining taxes }\end{array}$ & & Objective: Increase revenues from the mining sector & \\
\hline
\end{tabular}




\begin{tabular}{|c|c|c|c|}
\hline \multirow[t]{2}{*}{ Sector } & \multicolumn{2}{|r|}{ Measures } & \multirow[b]{2}{*}{$\begin{array}{l}\text { Timetable } \\
\text { End-June } 2010 . \\
\text { End-October } 2011 .\end{array}$} \\
\hline & 1. & $\begin{array}{l}\text { Strengthen tax administration in the mining sector: } \\
\text { - Strengthen the capacity of the specialized unit, including } \\
\text { by means of agreements with specialized audit } \\
\text { companies and international consulting firms. } \\
\text { Submit to Parliament draft legislation to transfer all } \\
\text { responsibility for tax administration in the mining sector } \\
\text { from the DGRAD to the new specialized unit in the DGE, } \\
\text { which becomes responsible for internal assessment, in } \\
\text { keeping with international best practice, for the audit and } \\
\text { collection of all taxes, fees, and royalties from the major } \\
\text { mining companies. }\end{array}$ & \\
\hline & 2. & $\begin{array}{l}\text { Starting in July 2009, conduct annual audits by the } \\
\text { Inspectorate General of Finance, six months after the year in } \\
\text { question, to verify that all tax revenue paid by the mining } \\
\text { companies have, in fact, been transferred to the general } \\
\text { account of the Treasury. }\end{array}$ & $\begin{array}{l}\text { End-December } \\
2010 .\end{array}$ \\
\hline & 3. & $\begin{array}{l}\text { In the context of strengthening the capacity of OFIDA, } \\
\text { establish centers of expertise for the principal mining exports } \\
\text { at Kasumbalesa, Sakania, Katanga, Goma and Bukavu. }\end{array}$ & End-June 2011. \\
\hline & 4. & $\begin{array}{l}\text { Strengthen monitoring of oil producers by subjecting them to } \\
\text { regular inspections (at least once a year) to check the } \\
\text { information contained in their declarations to the DGRAD and } \\
\text { the DGI. }\end{array}$ & Continuous. \\
\hline \multirow{7}{*}{$\begin{array}{l}\text { E. Management } \\
\text { of public finances }\end{array}$} & & Objective: Strengthen the management of public finances & \\
\hline & & $\begin{array}{l}\text { Adoption by the government of an action plan on reforms of } \\
\text { public finances. }\end{array}$ & Done. \\
\hline & 1. & $\begin{array}{l}\text { Law on public finances } \\
\text { - Submit to the Parliament the draft legislation. } \\
\text { - Adoption the by Parliament and promulgation of by the } \\
\text { President (Structural benchmark). } \\
\text { Adopt by the Council of Ministers decrees to implement } \\
\text { the law. }\end{array}$ & $\begin{array}{l}\text { Done. } \\
\text { Done } \\
\text { End-March } 2011 .\end{array}$ \\
\hline & 2. & $\begin{array}{l}\text { Adoption by the Council of Ministers of an action plan to } \\
\text { transfer areas of responsibility between the central } \\
\text { government and the provinces. }\end{array}$ & $\begin{array}{l}\text { End of December } \\
2010 .\end{array}$ \\
\hline & 3. & $\begin{array}{l}\text { Submit to the Parliament the draft audit report by the Audit } \\
\text { Office on the } 2008 \text { budget implementation. }\end{array}$ & Done. \\
\hline & 4. & $\begin{array}{l}\text { Adoption by parliament and promulgation by the President of } \\
\text { the new Public Procurement Code and its implementing } \\
\text { regulations: } \\
\text { Adopt and promulgate the law related to public procurement. } \\
\text { Sign the regulation. }\end{array}$ & $\begin{array}{l}\text { Done. } \\
\text { End-June } 2010 .\end{array}$ \\
\hline & 5. & $\begin{array}{l}\text { Reform the financing of tax collection agencies: } \\
\text { - Eliminate transfers to the three government tax collection } \\
\text { agencies and replace them with budget appropriations. }\end{array}$ & Done. \\
\hline
\end{tabular}




\begin{tabular}{|c|c|c|c|}
\hline \multirow[t]{5}{*}{ Sector } & \multicolumn{2}{|r|}{ Measures } & \multirow{2}{*}{$\begin{array}{c}\text { Timetable } \\
\text { End-January } 2011 .\end{array}$} \\
\hline & & $\begin{array}{l}\text { Replace performance bonuses with budget appropriations } \\
\text { covering operating and capital costs and with incentives } \\
\text { for officials in the tax collection agencies. }\end{array}$ & \\
\hline & 6. & Approve of general regulations on government accounting. & $\begin{array}{l}\text { End-December } \\
2010 .\end{array}$ \\
\hline & 7. & $\begin{array}{l}\text { Submit to the Parliament a draft organic law on the Audit } \\
\text { Office. }\end{array}$ & $\begin{array}{l}\text { End-December } \\
2010 .\end{array}$ \\
\hline & 7. & $\begin{array}{l}\text { Review the regulatory framework of the Office of the } \\
\text { Inspectorate General of Finance. }\end{array}$ & $\begin{array}{l}\text { End-December } \\
2010 .\end{array}$ \\
\hline $\begin{array}{l}\text { (ii) Budget } \\
\text { preparation }\end{array}$ & 1. & $\begin{array}{l}\text { Develop a medium-term budget framework: } \\
\text { - For the Ministry of Health and the Ministry of Education. } \\
\text { - In all ministries, in close cooperation with sector } \\
\text { ministries. }\end{array}$ & $\begin{array}{l}\text { End-September } \\
2010 . \\
\text { End-December } \\
2012 \text {. }\end{array}$ \\
\hline \multirow[t]{6}{*}{$\begin{array}{l}\text { (iii) Budget } \\
\text { execution }\end{array}$} & 1. & $\begin{array}{l}\text { Publish a decree by the Minister of Finance to oblige the } \\
\text { government to pay resident suppliers in the DRC in local } \\
\text { currency. }\end{array}$ & Done. \\
\hline & 2. & $\begin{array}{l}\text { Conduct an audit of all accounts of government entities at the } \\
\text { BCC and at commercial banks. }\end{array}$ & $\begin{array}{l}\text { End-December } \\
2010 .\end{array}$ \\
\hline & 3. & $\begin{array}{l}\text { Strengthen the computerized expenditure process by } \\
\text { improving the software and updating the hardware. }\end{array}$ & End-March 2011. \\
\hline & 4. & $\begin{array}{l}\text { Regularize domestic arrears: } \\
\text { - Prepare a plan to repay accumulated arrears as of the end } \\
\text { of 2008, based on the audit done by the Office of the } \\
\text { Inspector-General of Finance. (structural benchmark) } \\
\text { - Carry out the action plan to repay accumulated arrears as } \\
\text { of the end of } 2008 \text { gradually. }\end{array}$ & $\begin{array}{l}\text { Done. } \\
\text { End-January } 2011 .\end{array}$ \\
\hline & 5. & $\begin{array}{l}\text { Limit the use of the extraordinary expenses procedure: } \\
\text { Comply rigorously with the decree of December } 2008 \text {, } \\
\text { and halt the use of discretionary disbursements. } \\
\text { Implement a strategy to incorporate the procedure for } \\
\text { making urgent expenditures within the computerized } \\
\text { expenditure cycle. }\end{array}$ & $\begin{array}{l}\text { Done. } \\
\text { Done. }\end{array}$ \\
\hline & 6. & $\begin{array}{l}\text { Draw up a disbursement plan that conforms to the cash-flow } \\
\text { plan. }\end{array}$ & Continuous. \\
\hline \multirow[t]{2}{*}{$\begin{array}{l}\text { (iv) Accounting } \\
\text { system and budget } \\
\text { reports }\end{array}$} & 1. & $\begin{array}{l}\text { Finalize the implementation of double-entry bookkeeping at } \\
\text { the Directorate of the Treasury in the Ministry of Finance and } \\
\text { expand it to all revenue collection agencies. }\end{array}$ & $\begin{array}{l}\text { End of December } \\
2010 .\end{array}$ \\
\hline & 2. & $\begin{array}{l}\text { Production and publication of monthly tables (structural } \\
\text { benchmark): } \\
\text { - Generate and publish monthly budget execution tables } \\
\text { (reconciled with the TOFE, the BCC, and the monetary } \\
\text { statistics), no later than } 30 \text { days after the end of each } \\
\text { month. } \\
\text { Include externally financed expenditures (excluding } \\
\text { exceptional spending) in the monthly budget execution } \\
\text { tables (reconciled with the TOFE, the BCC, and the } \\
\text { monetary statistics), no later than } 3 \text { months after the end } \\
\text { of each month. }\end{array}$ & Continuous. \\
\hline
\end{tabular}




\begin{tabular}{|c|c|c|c|}
\hline Sector & & Measures & Timetable \\
\hline \multirow[t]{2}{*}{$\begin{array}{l}\text { (v) Payroll } \\
\text { administration }\end{array}$} & 1. & $\begin{array}{l}\text { Extend the Simplified Transitional System (PTS) for the } \\
\text { payroll to: } \\
\text { - all public officials and government employees } \\
\text { - all provinces }\end{array}$ & $\begin{array}{l}\text { End-December } \\
2010 . \\
\text { End-December } \\
2010 .\end{array}$ \\
\hline & 2. & $\begin{array}{l}\text { Harmonize the various civil service payroll administration } \\
\text { systems, particularly by revising the salary schedules } \\
\text { established under those systems. }\end{array}$ & $\begin{array}{l}\text { End-December } \\
2010 .\end{array}$ \\
\hline \multicolumn{4}{|c|}{ II. Monetary and financial policy } \\
\hline $\begin{array}{l}\text { A. Reform of the } \\
\text { central bank }\end{array}$ & & $\begin{array}{l}\text { Objective: Make the central bank independent and } \\
\text { efficient }\end{array}$ & \\
\hline \multirow[t]{3}{*}{$\begin{array}{l}\text { (i) Restructuring } \\
\text { of the central bank }\end{array}$} & 1. & $\begin{array}{l}\text { Restructure the National Mint } \\
\text { - } \quad \text { Evaluate assets of the Mint (building, equipment, human } \\
\text { resources). } \\
\text { - Create the restructuring committee of the Mint. } \\
\text { - } \quad \text { Adoption of a restructuring action plan. } \\
\text { Restructure the Mint. }\end{array}$ & $\begin{array}{l}\text { End-June } 2011 . \\
\text { End-May } 2010 . \\
\text { End-June } 2010 . \\
\text { End-June } 2011 .\end{array}$ \\
\hline & 2. & $\begin{array}{l}\text { Restructure (privatize) the central bank hospital: } \\
\text { - Create the restructuring committee of the hospital. } \\
\text { - } \quad \text { Adoption of a restructuring action plan. } \\
\text { - } \quad \text { Restructure the hospital. } \\
\end{array}$ & $\begin{array}{l}\text { End-May } 2010 . \\
\text { End-June } 2010 \\
\text { End - June } 2011 .\end{array}$ \\
\hline & 3. & $\begin{array}{l}\text { Reorganize the pension plan: } \\
\text { - Close off access to the old pension plan, so that it no } \\
\text { longer applies to staff members who are in active service } \\
\text { or to new staff. } \\
\text { - Appoint a pension committee. } \\
\text { - Inform all central bank staff about the main features of } \\
\text { the new pension plan. } \\
\text { - Administer the new pension plan and manage its } \\
\text { investments. }\end{array}$ & $\begin{array}{l}\text { End-January } 2011 . \\
\text { End-March } 2011 . \\
\text { End-July } 2011 . \\
\text { End-July } 2011 .\end{array}$ \\
\hline \multirow[t]{4}{*}{$\begin{array}{l}\text { (ii) Recapitaliza- } \\
\text { tion of the central } \\
\text { bank }\end{array}$} & 1. & $\begin{array}{l}\text { Adopt by the Council of Ministers the action plan to } \\
\text { recapitalize the central bank based on the recommendations of } \\
\text { the IMF technical assistance mission. }\end{array}$ & End-July 2010. \\
\hline & 2. & $\begin{array}{l}\text { Submit to the Parliament the draft law on the recapitalization } \\
\text { of the central bank. }\end{array}$ & End-October 2010. \\
\hline & 3. & $\begin{array}{l}\text { Adoption by the Council of Ministers of the budget needed to } \\
\text { recapitalize the central bank by issuing government securities. } \\
\text { Interest rates and maturities remain to be determined, } \\
\text { depending on market conditions. }\end{array}$ & $\begin{array}{l}\text { End-November } \\
2010 .\end{array}$ \\
\hline & 4. & Recapitalize the central bank. & End-June 2011. \\
\hline $\begin{array}{l}\text { B. Monetary } \\
\text { policy }\end{array}$ & & $\begin{array}{l}\text { Objective: Strengthen the central bank's capacity to } \\
\text { devise monetary and exchange policy }\end{array}$ & \\
\hline $\begin{array}{l}\text { (i) Issuing of } \\
\text { currency, and } \\
\text { quality of } \\
\text { currency in } \\
\text { circulation }\end{array}$ & 1. & $\begin{array}{l}\text { Increase the banknote processing capacity to } 100 \text { percent by } \\
\text { allocating the necessary resources to the Treasury Directorate: } \\
\text { - } 40 \text { percent; } \\
\text { - } 70 \text { percent; } \\
\text { - } \quad 100 \text { percent. }\end{array}$ & $\begin{array}{l}2009 \text { to } 2011 \\
\text { End-June } 2010 . \\
\text { End-December } \\
2010 . \\
\text { End -June } 2011 .\end{array}$ \\
\hline
\end{tabular}




\begin{tabular}{|c|c|c|c|}
\hline Sector & & Measures & Timetable \\
\hline \multirow[b]{3}{*}{$\begin{array}{l}\text { (ii) Foreign } \\
\text { exchange } \\
\text { operations }\end{array}$} & 2. & $\begin{array}{l}\text { Adopt a 3-year strategy to reform the currency issuance } \\
\text { function in the provinces, to improve the quality of currency } \\
\text { in circulation, in coordination with assistance from the IMF } \\
\text { expert. }\end{array}$ & Done. \\
\hline & 3. & $\begin{array}{l}\text { Issue coins in } 2011 \text { in accordance with the strategy developed } \\
\text { with the assistance of the IMF expert. }\end{array}$ & End-June 2011. \\
\hline & 1. & $\begin{array}{l}\text { Hold regular foreign exchange auctions and publish advance } \\
\text { notices on the Internet to maintain a continuing presence on } \\
\text { the foreign exchange market and assure the transparency of } \\
\text { market operations. }\end{array}$ & Ongoing (done). \\
\hline $\begin{array}{l}\text { C. Banking } \\
\text { supervision; } \\
\text { banking system }\end{array}$ & & $\begin{array}{l}\text { Objective: Strengthen banking supervision capacity and } \\
\text { improve the health of the banking system }\end{array}$ & \\
\hline \multirow{4}{*}{$\begin{array}{l}\text { (i) Banking } \\
\text { supervision } \\
\text { (ii) Banking } \\
\text { system }\end{array}$} & 1. & $\begin{array}{l}\text { Implement a new matrix of sanctions for noncompliance with } \\
\text { the banking supervision regulations. }\end{array}$ & End - June 2010. \\
\hline & 1. & $\begin{array}{l}\text { Perform audits to assess the quality of the loan portfolio of all } \\
\text { remaining banks, and establish a plan to restructure or } \\
\text { recapitalize them. }\end{array}$ & End-May 2010. \\
\hline & 2. & $\begin{array}{l}\text { Adopt a strategy to improve the health of commercial banks } \\
\text { based on the results of the March } 2009 \text { assessment undertaken } \\
\text { with technical assistance from IMF and World Bank experts. }\end{array}$ & Done. \\
\hline & 3. & Restructure a major commercial bank. & $\begin{array}{l}\text { Initial steps taken by } \\
\text { end-May } 2010 .\end{array}$ \\
\hline \multirow{3}{*}{$\begin{array}{l}\text { D. Accounting } \\
\text { and transparency } \\
\text { (i) Accounting and } \\
\text { audit operations } \\
\text { (ii) Transparency } \\
\text { and communica- } \\
\text { tions }\end{array}$} & & Objective: Improve accounting and transparency & \\
\hline & 1. & $\begin{array}{l}\text { Approve by the central bank's board of directors an action } \\
\text { plan to adopt and implement International Financial Reporting } \\
\text { Standards (IFRS). }\end{array}$ & $\begin{array}{l}\text { End-December } \\
2010 .\end{array}$ \\
\hline & 1. & $\begin{array}{l}\text { Not later than six months after the end of the financial year, } \\
\text { publish the central bank's financial statements and audit } \\
\text { reports, including the auditor's opinion. }\end{array}$ & Continuous. \\
\hline \multicolumn{4}{|c|}{ III. Other structural reforms } \\
\hline \multirow[t]{2}{*}{ A. Civil service } & 1. & Complete the survey of the civil service. & $\begin{array}{l}\text { End-December } \\
2011 .\end{array}$ \\
\hline & 2. & Complete the survey of the army and the police. & $\begin{array}{l}\text { End-December } \\
2011 .\end{array}$ \\
\hline \multirow{4}{*}{$\begin{array}{l}\text { B. Business } \\
\text { climate }\end{array}$} & 1. & Send to Parliament the OHADA membership treaty. & Done. \\
\hline & 2. & $\begin{array}{l}\text { Adopt by the government a commercial cod project, which } \\
\text { allows eliminating all tariff and nontariff barriers that are not } \\
\text { consistent with the international agreements ratified by the } \\
\text { DRC. }\end{array}$ & $\begin{array}{l}\text { End-November } \\
2010 .\end{array}$ \\
\hline & 3. & $\begin{array}{l}\text { Submit to Parliament a draft law on liberalizing the insurance } \\
\text { sector. }\end{array}$ & $\begin{array}{l}\text { End-November } \\
2010 .\end{array}$ \\
\hline & 4. & $\begin{array}{l}\text { Set up commercial courts in: } \\
\text { - the cities of Kisangani and Matadi; } \\
\text { - the remaining provinces. }\end{array}$ & $\begin{array}{l}\text { End-December } \\
2010 . \\
\text { End-December } \\
2011 .\end{array}$ \\
\hline
\end{tabular}




\begin{tabular}{|c|c|c|c|}
\hline Sector & \multicolumn{2}{|r|}{ Measures } & Timetable \\
\hline $\begin{array}{l}\text { C. Political } \\
\text { Decentralization }\end{array}$ & 1. & $\begin{array}{l}\text { Implement the 40-percent revenue transfer formula: } \\
\text { - transition period; } \\
\text { - implementation. }\end{array}$ & $\begin{array}{l}\text { Done. } \\
\text { Done. }\end{array}$ \\
\hline $\begin{array}{l}\text { (i) Classification } \\
\text { of levies and } \\
\text { charges for the } \\
\text { provinces and } \\
\text { ETDs }\end{array}$ & 1. & $\begin{array}{l}\text { Submit to Parliament a draft law on the classification of levies } \\
\text { and charges for the provinces and decentralized territorial } \\
\text { entities (ETDs). }\end{array}$ & $\begin{array}{l}\text { End-November } \\
2010 .\end{array}$ \\
\hline \multirow[t]{3}{*}{$\begin{array}{l}\text { (ii) Strengthen the } \\
\text { management of } \\
\text { public finances at } \\
\text { the provincial } \\
\text { level }\end{array}$} & 1. & $\begin{array}{l}\text { Simplify the budget classification of provincial } \\
\text { administrations: } \\
\text { - Revise the revenue and expenditure classification system. } \\
\text { - Introduce a simplified classification system for the } 2012 \\
\text { budget law for the provinces. }\end{array}$ & $\begin{array}{l}\text { End-November } \\
2010 . \\
\text { End-December } \\
2011 .\end{array}$ \\
\hline & 2. & $\begin{array}{l}\text { Develop and approve an action plan to strengthen capacity for } \\
\text { the management of public finances at the provincial level. }\end{array}$ & $\begin{array}{l}\text { End-September } \\
2010 .\end{array}$ \\
\hline & 3. & $\begin{array}{l}\text { In all provinces, implement a computerized expenditure } \\
\text { process and link it to the central government's expenditure } \\
\text { process. }\end{array}$ & $\begin{array}{l}\text { End-December } \\
2010 .\end{array}$ \\
\hline \multirow[t]{2}{*}{$\begin{array}{l}\text { D. Debt } \\
\text { management }\end{array}$} & 1. & $\begin{array}{l}\text { Execute the action plan for effective implementation of the } \\
\text { decree that centralizes external debt management in the } \\
\text { DGDP including all information concerning public debt as } \\
\text { well as all application measures. }\end{array}$ & $\begin{array}{l}\text { End-December } \\
2011 .\end{array}$ \\
\hline & 2. & $\begin{array}{l}\text { Effectively collect and centralize data on internal and external } \\
\text { public debt in the DGDP. }\end{array}$ & $\begin{array}{l}\text { End-December } \\
2010 .\end{array}$ \\
\hline \multirow[t]{3}{*}{ E. Transparency } & 1. & $\begin{array}{l}\text { Publication within } 60 \text { days: } \\
\text { - Of mining sector partnership contracts between public } \\
\text { and private enterprises (including information on signing } \\
\text { bonuses, taxation system, private shareholders, and } \\
\text { members of the board of directors. (structural } \\
\text { benchmark) } \\
\text { Of the negotiation results between mining companies and } \\
\text { the government regarding the review of mining contracts. }\end{array}$ & Continuous. \\
\hline & 2. & $\begin{array}{l}\text { Implement the Extractive Industries Transparency Initiative } \\
\text { (EITI). }\end{array}$ & $\begin{array}{l}\text { End-September } \\
2010 .\end{array}$ \\
\hline & 3. & $\begin{array}{l}\text { Establish an independent watchdog agency on corruption: } \\
\text { Prepare and adopt the draft law to establish an independent } \\
\text { watchdog agency on corruption. } \\
\text { Submit to the Parliament by the Government the draft law. }\end{array}$ & $\begin{array}{l}\text { End-October } 2010 \\
\text { End-November } \\
2010 .\end{array}$ \\
\hline \multirow[t]{2}{*}{ F. Statistics } & 1. & $\begin{array}{l}\text { Submission by the government of the draft statistics law to the } \\
\text { Parliament. }\end{array}$ & $\begin{array}{l}\text { Adopted by the } \\
\text { government in } \\
\text { January } 2010 \text {. }\end{array}$ \\
\hline & 2. & $\begin{array}{l}\text { Revision of national accounts statistics: } \\
\text { - Finalize the estimated national accounts data (SNA 1993) } \\
\text { for } 2005 \text { and } 2006 . \\
\text { - Finalize the estimated national accounts data (SNA 1993) } \\
\text { for } 2007 \text {. } \\
\text { - Finalize the estimated national accounts data (SNA 1993) } \\
\text { for } 2008 .\end{array}$ & $\begin{array}{l}\text { Done. } \\
\text { End-September } \\
2010 . \\
\text { End-December } \\
2010 .\end{array}$ \\
\hline
\end{tabular}




\begin{tabular}{|c|c|c|c|}
\hline \multirow[t]{2}{*}{ Sector } & \multicolumn{2}{|r|}{ Measures } & Timetable \\
\hline & & $\begin{array}{l}\text { - Have the government adopt the national accounts in } \\
\text { accordance with SNA } 1993 \text {. }\end{array}$ & End-March 2011 \\
\hline & 3. & $\begin{array}{l}\text { Finalization and adoption by the government the national } \\
\text { statistical development strategy (SNDS). }\end{array}$ & $\begin{array}{l}\text { End-September } \\
2010 .\end{array}$ \\
\hline \multirow[t]{2}{*}{$\begin{array}{l}\text { G. Reform of } \\
\text { State enterprises }\end{array}$} & 1. & $\begin{array}{l}\text { Develop and submit to the Council of Ministers the social } \\
\text { programs for the targeted enterprises: } \\
\text { - } \quad \text { SNCC and REGIDESO. } \\
\text { - } \quad \text { SNEL. } \\
\text { - ONATRA and RVA. }\end{array}$ & $\begin{array}{l}\text { End-July } 2010 \\
\text { Done. } \\
\text { End-December } \\
\text { 2010. } \\
\text { End-June } 2011 .\end{array}$ \\
\hline & 2. & $\begin{array}{l}\text { Evaluate cross-debts between the government and State } \\
\text { enterprises, and between State enterprises: } \\
\text { - Select the consultant. } \\
\text { - Complete the evaluation report. } \\
\text { - Adopt the action plan by the government. }\end{array}$ & $\begin{array}{l}\text { Done. } \\
\text { End-November } \\
2010 . \\
\text { End-December } \\
2010 .\end{array}$ \\
\hline
\end{tabular}




\section{APPENDIX I \\ ATTACHMENT II \\ TRANSLATED FROM FRENCH \\ DEMOCRATIC REPUblic OF THE CONGo \\ TECHNiCAL MEMORANDUM OF UNDERSTANDING \\ on Program IMPLEMENTATION}

Kinshasa, June 15, 2010

1. This memorandum updates the Technical Memorandum of Understanding (TMU) accompanying IMF Country Report No. 10/88. It defines the quantitative targets that will be used to assess the performance by the Democratic Republic of the Congo under the program supported by the Extended Credit Facility (ECF). It also specifies the content and frequency of the data needed for program monitoring. The updated TMU is applicable from July 1, 2010 onwards while that accompanying IMF Country Report No. 10/88 will continue to apply until June 30, 2010, including the definitions of the quantitative targets and adjustors. Unless otherwise indicated, all the quantitative targets are measured in terms of cumulative changes since the beginning of the year (January 1). Variables denominated in U.S. dollars will be converted to Congo francs by using the program exchange rate of CGF 639.32 per U.S. dollar. Variables denominated in currencies other than the U.S. dollar (excluding the SDRs and Euro) will first be converted to U.S. dollars at the end-period US\$/currency exchange rate. Variables denominated in SDR will be valued at the program exchange rate of CGF 994.02 per SDR. Variables denominated in euro will be valued at the program exchange rate of CGF 905.07 per Euro.

2. Institutional coverage: The central government comprises all units of government that exercise authority over the entire economic territory. However, unless otherwise indicated for the purposes of this memorandum, the central government does not include nonprofit organizations controlled and financed by the central government. The banking system is understood to mean the Central Bank of the Congo (BCC) as well as existing or newly licensed commercial banks.

\section{Quantitative Performance Criteria}

3. Quantitative performance criteria are established end-December 2010 and indicative targets for end-September 2010 with regard to the following variables: ${ }^{1}$

\footnotetext{
${ }^{1}$ The end-June performance criteria are those established in the MEFP accompanying IMF Country Report No. $10 / 88$.
} 
- Changes in the net foreign assets of the BCC;

- $\quad$ Changes in the net domestic assets of the BCC;

- Changes in net banking system credit to the government (central government);

- Payments of government expenditures (including emergency expenditures) by the BCC without the prior authorization according to proper budgetary procedures by the Ministries of Budget and Finance;

- $\quad$ Nonconcessional medium- and long-term foreign loans contracted or guaranteed by the central government, local governments, or the $\mathrm{BCC}$;

- Nonconcessional short-term foreign loans contracted or guaranteed by the State, local governments, or the $\mathrm{BCC}$; and

- The accumulation of external payment arrears.

\section{A. Floors on the Net Foreign Assets of the BCC}

4. Definition: Net foreign assets (NFA) are defined as the difference between the BCC gross international reserves and its total liabilities. Gross foreign assets are defined as the sum of the following items: (i) monetary gold holdings of the BCC; (ii) SDR holdings; (iii) convertible claims on nonresidents, such as foreign deposits and foreign securities. The following items are excluded from the definition of gross reserves: claims on residents in foreign exchange, nonconvertible currency holdings, and reserves that are encumbered or pledged in one form or another, including but not limited to reserve assets used as collateral or security for foreign third-party liabilities. Foreign liabilities are all BCC foreign exchange liabilities to nonresidents (including SDR allocations), including the IMF.

5. The following adjustments will be made to the NFA floors.

- Balance of payments support (BPS): NFA will be adjusted (i) upward by an amount equivalent to total BPS in excess of the programmed levels, (ii) downward by (a) an amount equivalent to the lesser of total shortfalls of BPS (excluding that from the IMF) relative to programmed levels and US\$50 million by end-September 2010 and (b) an amount equivalent to the lesser of total shortfalls of BPS (excluding that from the IMF) relative to programmed levels and US\$100 million by end-December 2010; and (iii) downward by an amount equivalent to any shortfall in debt relief under the Heavily indebted Poor Countries (HIPC) Initiative and the Multilateral Debt Relief Initiative (MDRI) from the IMF.

- Scheduled external debt service: NFA will be adjusted (i) upward by an amount equivalent to under payment of debt service relative to programmed amounts; (ii) downward by an amount equivalent to the excess of external debt service payments relative to programmed amounts. 
- Signing bonus from the Sino-Congolese Cooperation Agreement (SCCA): NFA will be adjusted (i) upward by an amount equivalent to total disbursement of signing bonus from SCCA in excess of the programmed levels; (ii) downward by an amount equivalent to total shortfalls in the disbursement of signing bonus from SCCA relative to programmed levels.

- Privatization proceeds in convertible currencies (PPCC): NFA will be adjusted upward by an amount equivalent to total PPCC in excess of the programmed levels.

6. Definition: BPS includes (all grants and loans) minus (grants and loans for externally financed projects) plus (debt relief granted by the IMF under the HIPC initiative and MDRI). External financing for the National Disarmament, Demobilization, and Reintegration Program (DDR) is defined as externally financed projects and is therefore not included in the definition of BPS.

7. Definition: scheduled external debt service payments (excluding those to the IMF) are defined as debt service due (principal and interest) minus debt relief (excluding debt relief offered by the IMF).

\section{B. Ceilings on the Net Domestic Assets of the BCC}

8. Definition: The net domestic assets (NDA) of the BCC are defined as base money (see 918 below) minus NFA. Based on this definition, the NDA of the BCC include: (i) net credit to the government (central government) (see paragraph 10 below); (ii) credit to the private sector; (iii) credit to public enterprises; (iv) credit to commercial banks; and (v) other net assets.

9. The following adjustments will be made to the NDA ceilings.

- BPS: NDA will be adjusted (i) downward by an amount equivalent to total BPS in excess of the programmed level; (ii) upward by (a) an amount equivalent to the lesser of total shortfalls of BPS (excluding that from the IMF) relative to programmed levels and CGF 31,966 million by end-September 2010 and (b) an amount equivalent to the lesser of total shortfalls of BPS (excluding that from the IMF) relative to programmed levels and CGF 63,932 million by end-December 2010; and (iii) upward by an amount equivalent to any shortfall in debt relief under the HIPC/MDRI from the IMF.

- Scheduled external debt service: NDA will be adjusted (i) downward by an amount equivalent to under payment of debt service relative to programmed amounts; (ii) upward by an amount equivalent to the excess of external debt service payments relative to programmed amounts. 
- $\quad$ Signing bonus from the SCCA: NDA will be adjusted (i) downward by an amount equivalent to total disbursement of signing bonus from SCCA in excess of the programmed levels; (ii) upward by an amount equivalent to total shortfalls in the disbursement of signing bonus from SCCA relative to programmed levels.

- Privatization proceeds: NDA will be adjusted downward by the total amount of privatization proceeds (including PPCC) in excess of the programmed level.

\section{Ceiling on Net Banking System Credit to the Government}

Definition: Net banking system credit to the government (NCG) is defined as the sum of net $\mathrm{BCC}$ and commercial bank claims on the central government, plus the BCC's net cash deficit. For purposes of program monitoring, government deposits related to externally financed projects are excluded from NCG. External budget support (BPS excluding balance of payment support from the IMF) will be converted to domestic currency by using the market exchange rate prevailing at the time of the disbursement.

10. The following adjustments will be made to the NCG ceiling.

- BPS: NCG will be adjusted (i) downward by an amount equivalent to total BPS (excluding that from the IMF) in excess of the programmed level, and (ii) upward by (a) an amount equivalent to the lesser of total shortfalls of BPS (excluding that from the IMF) relative to programmed levels and CGF 48,050 million by end-September 2010 and (b) an amount equivalent to the lesser of total shortfalls of BPS (excluding that from the IMF) relative to programmed levels and CGF 96,100 million by end-December 2010.

- Debt service payment: NCG will be adjusted (i) downward by an amount equivalent to under payment of debt service relative to programmed amounts; (ii) upward by an amount equivalent to the excess of external debt service payments relative to programmed amounts.

- Signing bonus from the SCCA: NCG will be adjusted (i) downward by an amount equivalent to total disbursement of signing bonus from SCCA in excess of the programmed levels; (ii) upward by an amount equivalent to total shortfalls relative to programmed levels in the disbursement of signing bonus from SCCA.

- Privatization proceeds: the NCG ceiling will be adjusted downward by an amount equivalent to total privatization proceeds in excess of the programmed levels. 


\section{Ceilings on New Nonconcessional Loans Contracted or Guaranteed by the Central Government, Local Governments, or the BCC}

11. Definition: With regard to contracted or guaranteed debt, the central government is defined as all units of government that exercise authority over the entire economic territory, including nonprofit organizations controlled and financed by the central government.

12. Definition: Debt is defined as set out in Executive Board Decision No. 12274, Point 9, as revised on August 3, 2009 (see Annex). ${ }^{2}$ For program purposes, external debt is measured on a gross basis using the residency criterion.

\section{E. Ceilings on New Medium-and Long-term Nonconcessional Loans Contracted or Guaranteed by the Central Government, Local Governments, or the BCC}

13. Definition: nonconcessional debt is defined as all loans with a grant element of less than 35 percent, calculated as the difference between the present value (PV) of the debt and its nominal value, expressed as a percentage of the nominal value of the debt. The PV of the debt at the time it is contracted is calculated by discounting future flows of payable debt service. The discount rates used for that purpose are the commercial interest reference rates (CIRR) specific to each currency, published by the OECD. The average ten-year CIRRs will be used to calculate the PV of debt having a maturity of at least 15 years, and six-month average CIRRs for loans with shorter maturity. For the purposes of the program, the most recent CIRRs published by the OECD will be used to assess concessionality of loans.

14. Definition: the limit on nonconcessional medium- and long-term loans applies to contracted or guaranteed debt and liabilities for which the equivalent value has not been received. It excludes (i) the use of Fund resources; (ii) lending from the World Bank, the African Development Bank, and the International Fund for Agricultural Development; (iii) debts incurred to restructure, refinance, or prepay existing debts, to the extent that such debt is incurred on more favorable terms than the existing debt; and; (iv) concessional debts.

15. Definition: the guarantee of a debt arises from any explicit legal obligation of the central government, the $\mathrm{BCC}$, or other agencies on behalf of the central government to service a loan in the event of nonpayment by the recipient (involving payments in cash or in kind), or indirectly through any other obligation of the central government, the BCC, or other agencies on behalf of the central government to finance a shortfall incurred by the loan recipient.

\footnotetext{
${ }^{2}$ http://www.imf.org/external/np/pp/eng/2009/082009.pdf .
} 


\section{F. Ceilings on New Nonconcessional Short-term Loans Contracted or Guaranteed by the Central Government, Local Governments, or the BCC}

16. Definition: short-term debt is defined as debt having an initial maturity of one year or less, with the exception of normal import credits having a maturity of up to one year, ${ }^{3}$ including overdraft positions and debt owed or guaranteed by the government or the BCC.

\section{G. Ceiling on the Accumulation of External Payment Arrears}

17. Definition: external payment arrears include external debt service obligations (principal and interest) that were not paid on the contractual due date. The ceiling on new external payment arrears applies continuously throughout the period covered by the PRGF arrangement. It does not apply to external payment arrears in process of renegotiation or to cases in which the creditor has agreed to the suspension of payments pending the outcome of negotiations.

\section{QuANTITATIVE INDICATIVE TARGETS}

18. The indicative targets pertain to: (i) base money; (ii) the non-accumulation of wage arrears; and (iii) the domestic fiscal balance.

\section{A. Ceilings on Base Money}

19. Definition: base money is defined as the sum of (i) currency outside banks; (ii) cash holdings of commercial banks; (iii) deposits of commercial banks with the BCC; (iv) private sector deposits with the BCC; (v) deposits of public enterprises with the BCC; and (vi) foreign exchange deposits and provisions for imports with the BCC.

\section{B. Ceilings on the Accumulation of Wage Arrears}

20. Definitions: Wage arrears are defined as approved personnel expenses that have not been paid for 30 days. Wages include the total compensation paid employees (civil service; including permanent benefits). These arrears will be valued on a cumulative basis from January 1, 2009.

\section{Ceiling on the Domestic Fiscal Balance}

21. Definitions: the domestic fiscal balance is defined as (domestic revenue) minus (domestically financed expenditure). Domestic revenue is defined as (total revenue and grants) minus (grants) minus (signing bonus from the SCCA). Domestically financed

\footnotetext{
${ }^{3}$ A financing arrangement for imports is considered to be "normal" when the credit is self-liquidating.
} 
expenditure is defined as (total expenditure and net lending) minus (externally financed investments) minus (foreign interest payments) plus (the BCC's operating deficit) plus (the net accumulation of domestic arrears).

22. The following adjustments will apply to the ceiling on the domestic fiscal balance:

- BPS: The floors on the domestic fiscal balance will be adjusted upward by an amount equivalent to the excess of total shortfall of BPS (excluding that from the IMF) relative to programmed levels over CGF 48,050 million by end-September 2010 and over CGF 96,100 million by end-December 2010.

\section{Consultation Clause}

23. In the event that revenue exceeds the programmed amounts during the program period, the authorities will consult the IMF before allocating any surplus revenue to additional expenditure. Further, the authorities will consult with the IMF before implementing any revisions to the policies contained in the MEFP.

\section{Data to be Reported for Program Monitoring Purposes}

24. The authorities of the DRC will provide IMF staff with the data needed to monitor the program within the prescribed time limits, as indicated in the following table. 
Summary of Data to be Reported

\begin{tabular}{|c|c|c|c|}
\hline Information & Responsible entity & Frequency & $\begin{array}{l}\text { Reporting } \\
\text { deadline }\end{array}$ \\
\hline $\begin{array}{l}\text { Volume of foreign exchange purchases and } \\
\text { sales on the interbank market }\end{array}$ & $\mathrm{BCC}$ & Daily & One day \\
\hline $\begin{array}{l}\text { Volume of BCC purchases and sales on the } \\
\text { interbank market }\end{array}$ & $\mathrm{BCC}$ & Daily & One day \\
\hline $\begin{array}{l}\text { Average CGF/USD reference exchange } \\
\text { rate on the interbank market }\end{array}$ & $\mathrm{BCC}$ & Daily & One day \\
\hline $\begin{array}{l}\text { Average CGF/USD reference exchange } \\
\text { rate }\end{array}$ & $\mathrm{BCC}$ & Daily & One day \\
\hline $\begin{array}{l}\text { Average CGF/USD reference exchange } \\
\text { rate offered by commercial banks to their } \\
\text { customers }\end{array}$ & $\mathrm{BCC}$ & Daily & One day \\
\hline $\begin{array}{l}\text { Average CGF/USD reference exchange } \\
\text { rate used by exchange bureaus }\end{array}$ & $\mathrm{BCC}$ & Daily & One day \\
\hline Integrated monetary survey & $\mathrm{BCC}$ & Monthly & Two weeks \\
\hline $\mathrm{BCC}$ balance sheet & $\mathrm{BCC}$ & Monthly & One week \\
\hline Monetary survey of retail banks & $\mathrm{BCC}$ & Monthly & Two weeks \\
\hline Structure of retail banks' interest rates & $\mathrm{BCC}$ & Monthly & Two weeks \\
\hline $\begin{array}{l}\text { Reserves (voluntary and required) of retail } \\
\text { banks }\end{array}$ & $\mathrm{BCC}$ & Daily & One day \\
\hline $\begin{array}{l}\text { Volume of CGF transactions on the } \\
\text { interbank market }\end{array}$ & $\mathrm{BCC}$ & Daily & One day \\
\hline $\begin{array}{l}\text { Outstanding central bank claims on retail } \\
\text { banks }\end{array}$ & $\mathrm{BCC}$ & Daily & One day \\
\hline $\begin{array}{l}\text { Foreign exchange sales (including through } \\
\text { auctions) by the BCC }\end{array}$ & $\mathrm{BCC}$ & Weekly & One week \\
\hline $\begin{array}{l}\text { Outstanding and new issues of central bank } \\
\text { bills (BTR) }\end{array}$ & $\mathrm{BCC}$ & Weekly & One day \\
\hline Change in the free reserves of banks & $\mathrm{BCC}$ & Weekly & One day \\
\hline Structure of BCC interest rates & $\mathrm{BCC}$ & Monthly & One week \\
\hline Consumer price index & $\mathrm{BCC}$ & Weekly & One week \\
\hline $\begin{array}{l}\text { Retail banks' financial soundness } \\
\text { indicators }\end{array}$ & $\mathrm{BCC}$ & Monthly & Two weeks \\
\hline $\begin{array}{l}\text { Commodity exports (value and volume), } \\
\text { imports (value and volume) and domestic } \\
\text { production indicators }\end{array}$ & $\mathrm{BCC}$ & Monthly & Three weeks \\
\hline $\begin{array}{l}\text { Implementation of the BCC foreign } \\
\text { exchange cash flow plan }\end{array}$ & $\mathrm{BCC}$ & Weekly & One week \\
\hline
\end{tabular}


Summary of Data to be Reported (Concluded)

\begin{tabular}{|c|c|c|c|}
\hline Information & Responsible entity & Frequency & $\begin{array}{l}\text { Reporting } \\
\text { deadline }\end{array}$ \\
\hline $\begin{array}{l}\text { Implementation of the BCC domestic } \\
\text { currency cash flow plan }\end{array}$ & $\mathrm{BCC}$ & Monthly & One week \\
\hline $\begin{array}{l}\text { Amounts and holders of promissory notes } \\
\text { (bills) guaranteed by the BCC }\end{array}$ & $\mathrm{BCC}$ & Monthly & Three weeks \\
\hline $\begin{array}{l}\text { Evolution of Commitment Plan and } \\
\text { Treasury Plan Implementation }\end{array}$ & $\mathrm{MF} / \mathrm{MB}$ & Weekly & One day \\
\hline $\begin{array}{l}\text { Implementation of the government cash } \\
\text { flow plan }\end{array}$ & MF & Monthly & Two weeks \\
\hline $\begin{array}{l}\text { Amount, terms, holders, and stock of } \\
\text { promissory notes (bills) }\end{array}$ & $\mathrm{MF} / \mathrm{BCC}$ & Monthly & Three weeks \\
\hline $\begin{array}{l}\text { Breakdown of customs and excise } \\
\text { revenues }\end{array}$ & MF & Monthly & Four weeks \\
\hline Breakdown of direct and indirect taxes & MF & Monthly & Four weeks \\
\hline Breakdown of nontax revenues & MF & Monthly & Four weeks \\
\hline $\begin{array}{l}\text { Projected expenditure commitment } \\
\text { schedule }\end{array}$ & MB & Quarterly & Two weeks \\
\hline Budgetary monitoring statement (ESB) & MB & Monthly & Two weeks \\
\hline Approved wage bill by type of beneficiary & MF & Monthly & Three weeks \\
\hline Wage bill paid by type of beneficiary & $\mathrm{MF}$ & Monthly & Three weeks \\
\hline Compensated employees by category & MF & Monthly & Three weeks \\
\hline Civil service wage scale & MF & $\begin{array}{l}\text { In the event of } \\
\text { change }\end{array}$ & Three weeks \\
\hline $\begin{array}{l}\text { Amounts of emergency spending, amounts } \\
\text { approved by the emergency spending } \\
\text { committee, amounts adjusted and paid by } \\
\text { the BCC }\end{array}$ & $\mathrm{MF} / \mathrm{BCC}$ & Monthly & Three weeks \\
\hline Privatization receipts & $\mathrm{MF} / \mathrm{BCC}$ & Monthly & Three weeks \\
\hline $\begin{array}{l}\text { Public sector domestic debt by category } \\
\text { and by creditor }\end{array}$ & MF & Monthly & Three weeks \\
\hline $\begin{array}{l}\text { Loan contracts for any new external debt } \\
\text { contracted or guaranteed by the central } \\
\text { government, the BCC and local } \\
\text { governments }\end{array}$ & $\mathrm{MF} / \mathrm{BCC}$ & Monthly & Three weeks \\
\hline $\begin{array}{l}\text { Budget execution monitoring table } \\
\text { showing Annual Treasury and } \\
\text { Commitment Plans and all stages of } \\
\text { expenditure execution through payments. }\end{array}$ & $\mathrm{MF} / \mathrm{MB}$ & Weekly & Three days \\
\hline $\begin{array}{l}\text { Price Waterhouse Coopers audit reports, } \\
\text { indicating any adjustments made to data } \\
\text { reported at test dates }\end{array}$ & $\mathrm{BCC}$ & & One week \\
\hline
\end{tabular}

reported at test dates 
Annex

\section{Definition of debt}

(a) For the purpose of this guideline, the term "debt" will be understood to mean a current, i.e., not contingent, liability, created under a contractual arrangement through the provision of value in the form of assets (including currency) or services, and which requires the obligor to make one or more payments in the form of assets (including currency) or services, at some future point(s) in time; these payments will discharge the principal and/or interest liabilities incurred under the contract. Debts can take a number of forms, the primary ones being as follows:

(i) loans, i.e., advances of money to the obligor by the lender made on the basis of an undertaking that the obligor will repay the funds in the future (including deposits, bonds, debentures, commercial loans and buyers' credits) and temporary exchanges of assets that are equivalent to fully collateralized loans under which the obligor is required to repay the funds, and usually pay interest, by repurchasing the collateral from the buyer in the future (such as repurchase agreements and official swap arrangements);

(ii) suppliers' credits, i.e., contracts where the supplier permits the obligor to defer payments until some time after the date on which the goods are delivered or services are provided; and

(iii) leases, i.e., arrangements under which property is provided which the lessee has the right to use for one or more specified period(s) of time that are usually shorter than the total expected service life of the property, while the lessor retains the title to the property. For the purpose of the guideline, the debt is the present value (at the inception of the lease) of all lease payments expected to be made during the period of the agreement excluding those payments that cover the operation, repair or maintenance of the property, and

(b) Under the definition of debt set out in point (a) above, arrears, penalties, and judicially awarded damages arising from the failure to make payment under a contractual obligation that constitutes debt are debt. Failure to make payment on an obligation that is not considered debt under this definition (e.g., payment on delivery) will not give rise to debt. 
INTERNATIONAL MONETARY FUND

DEMOCRATIC REPUBLIC OF THE CONGO

Democratic Republic of the Congo-First Review Under the Three-Year Arrangement Under the Extended Credit Facility and Financing Assurances Review

\section{Informational Annex}

Prepared by the African Department

June 15,2010

Relations with the Fund. Describes financial and technical assistance from the Fund and provides information on the safeguards assessment and exchange rate system. Outstanding purchases and loans amounted to SDR 499.52 million (93.72 percent of quota) as of May 31, 2010.

Joint Managerial Action Plan. Describes the IMF's and World Bank Group's work program.

Statistical Issues. Provides assessment of data adequacy for surveillance and program monitoring purposes.

Table of Common Indicators for Surveillance. Provides information on statistical data for surveillance purpose. 
Relations with the Fund

Relations with the World Bank Group......................................................................... 59

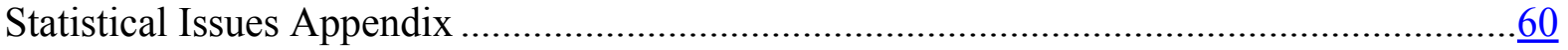

Table of Common Indicators Required for Surveillance ................................................... 


\section{Democratic RePUblic OF THE CONGo RELATIONS WITH THE FUND \\ (As of May 31, 2010)}

I. Membership Status: Joined September 28, 1963; Article VIII

II. General Resources Account:

Quota

Fund holdings of currency

III. SDR Department:

Net cumulative allocation

Holdings

IV. Outstanding Purchases and Loans:

ESF RAC loan

ECF Arrangement

$\begin{array}{cc}\text { SDR Million } & \begin{array}{c}\text { Percent of } \\ \text { Quota }\end{array} \\ 533.00 & 100.00 \\ 533.00 & 100.00\end{array}$

SDR Million

Percent of Quota

510.86

100.0

366.27

68.72

$\begin{array}{cc}\text { SDR Million } & \begin{array}{c}\text { Percent of } \\ \text { Quota }\end{array} \\ 133.25 & 25.00 \\ 366.27 & 68.72\end{array}$

V. Latest Financial Arrangements:

$\begin{array}{lcc}\text { Type } & \begin{array}{c}\text { Approval } \\ \text { Date }\end{array} & \begin{array}{c}\text { Expiration } \\ \text { Date }\end{array} \\ \text { ECF } & \text { Dec 11, 2009 } & \text { Dec 10,2012 } \\ \text { ECF } & \text { June 12, 2002 } & \text { Mar 31, 2006 } \\ \text { Stand-By } & \text { June 09, 1989 } & \text { June 08, 1990 }\end{array}$

\section{Amount Approved (SDR Million)}

$$
\begin{aligned}
& 346.45 \\
& 580.00 \\
& 116.40
\end{aligned}
$$

Amount Drawn (SDR Million)

49.49

553.47

75.00

VI. Projected Payments to Fund:

(SDR million; based on current use of resources and present holdings of SDRs):

Forthcoming

\begin{tabular}{lrrrrr}
\cline { 2 - 6 } & $\mathbf{2 0 1 0}$ & $\mathbf{2 0 1 1}$ & $\mathbf{2 0 1 2}$ & $\mathbf{2 0 1 3}$ & $\mathbf{2 0 1 4}$ \\
Principal & 94.68 & 110.69 & 68.69 & 24.03 & 26.68 \\
Charges/interest & 0.22 & 0.33 & 0.97 & 0.86 & 0.80 \\
Total & 94.90 & 111.02 & 69.66 & 24.88 & 27.48
\end{tabular}


VII. Implementation of HIPC Initiative:

I. Commitment of HIPC assistance

Enhanced Framework

Decision point date

July 2003

Assistance committed by all creditors (US\$ millions)

$6,311.00$

of which: IMF assistance (US\$ millions)

318.90

(SDR equivalent millions)

228.30

Completion point date

Floating

II. Disbursement of IMF assistance (SDR millions)

Assistance disbursed to the member

49.05

Interim assistance

49.05

Completion point balance

Additional disbursement of interest income

Total disbursement

49.05

VIII. Implementation of MDRI Assistance: Not applicable

IX. Exchange Rate Arrangement:

The currency of the Democratic Republic of the Congo is the Congo franc which, since May 26, 2001, has been freely floating. On June 15, 2010, the rate was US $\$ 1=C F 901$.

Effective February 10, 2003, the DRC accepted the obligations of Article VIII, Sections 2 (a), 3, and 4, of the Fund's Articles of Agreement; however, the DRC maintains measures that give rise to one restriction and one multiple currency practice subject to Fund approval. The exchange restriction involves an outstanding net debt position against other contracting members under the inoperative regional payments agreement with the Economic Community of the Great Lakes Countries. The multiple currency practice relates to a fixed exchange rate set quarterly applying to transactions through the bilateral payments agreement with Zimbabwe.

\section{Last Article IV Consultation:}

(a) Consultations with the Democratic Republic of the Congo are held in accordance with the provisions of the decision on consultation cycles approved on July 15, 2002.

(b) The last Article IV consultation was concluded by the Executive Board on December11, 2009. 
XI. Safeguards Assessment:

An update safeguards assessment of the central bank (BCC) was completed in April 2010. It found that most of 2008 recommendations have been implemented. However, significant risks remain. The central bank lacks independence from the government and its recapitalization requires urgent action. The BCC authorities agreed broadly with the priority recommendations of the assessment and some progress towards their implementation has been made.

\section{Technical Assistance:}

STA

FY 2004 - Multisector statistics

FY 2010 - Monetary statistics

\section{MCM}

\section{Capacity building assistance to the central bank}

Strengthening capacity in Anti-Money Laundering/Combating Financial Terrorism (AML/CFT)

FY 2005 - One short visit

FY 2006 - Two short visits

Strengthening capacity in monetary policy implementation, exchange operations, and liquidity management

FY 2005 - Three short visits

FY 2006 - Two short visits

FY 2007 - Seven short visits

FY 2008 - Three short visits

FY 2009 - One short visit

\section{Restructuring and reorganization of the central bank}

FY 2009 - One short visit

FY 2010 - One short visit

\section{Strengthening capacity in banking supervision and financial sector development}

FY 2005 - One short visit on bank supervision and regulation

FY 2008 - Two short visits on bank supervision and regulation

FY 2010 - Three short visits on bank supervision and regulation

FY 2006 - One short visit on internal audit

FY 2008 - One short visit on internal audit

FY 2007 - Action plan for developing the financial system

FY 2008 - One short visit on TA assessment 


\section{FAD}

Public financial management

FY 2007 - Short visit

FY 2009 - Short visit

Revenue administration

FY 2004 - Two short visits

FY 2005 - One short visit

FY 2006 - One short visit

FY 2007 - One short visit

FY 2007 - One short visit

FY 2009 - One short visit

Fiscal decentralization

FY 2005 - Short visit

XIII. Long-term Resident Experts:

Expenditure management

Advisor to Governor of $\mathrm{BCC}$

Capacity building of BCC

Statistics
Mr. Catalan

Mr. Nguenang

Mr. Akumchi

Mr. Bremeersch

Mr. Fossat

Mr. Nyssens

Mr. d'Ambrières

Mr. Pauwels

Mr. Métreau

Mr. Gbossa
Jan. 2002-June 2004

Sept. 2004-July 2008

Aug. 2008 - July 2009

Jan. 2002-Jan. 2004

Sept. 2005

Jan. 2004-Jan. 2005

Feb. 2006-June 2006

Sept. 2004-Mar. 2007

Oct. 2004

Dec. 2005-08

XIV. Resident Representative: Mr. Jahjah assumed his duties as Resident Representative in January 2009. 


\section{Democratic Republic of the Congo: \\ Relations with the World Bank Group \\ Joint Managerial Action Plan (JMAP) \\ (As of June 1, 2010)}

1. The IMF and World Bank Democratic Republic of Congo teams discussed a joint managerial action plan under this initiative.

2. The World Bank's work program entails moving forward in FY11 with a Development Policy Operation (DPO) to provide US $\$ 100$ million in budget support, and a Country Economic Memorandum (CEM) that will focus on constraints to growth.

3. The IMF's work program entails three year arrangement under the Extended Credit Facility (ECF) approved by the IMF Executive Board on December 11, 2009.

4. Both institutions are supporting an FSAP that will take place during FY11.

\begin{tabular}{|c|c|c|c|}
\hline Title & Products & Provisional timing of missions & Actual or Expected delivery date \\
\hline \multicolumn{4}{|c|}{ A. Mutual information on relevant work program } \\
\hline \multirow[t]{3}{*}{$\begin{array}{l}\text { World Bank work program in } \\
\text { the next } 12 \text { months }\end{array}$} & $\begin{array}{l}\text { Development Policy Operation } \\
\text { that may provide } \\
\text { US } \$ 100 \text { million in budget } \\
\text { support }\end{array}$ & $\begin{array}{l}\text { September 2010-November } \\
2010\end{array}$ & December 2010 \\
\hline & $\begin{array}{l}\text { Diagnostic Trade Integration } \\
\text { Study }\end{array}$ & June 2010 & June/July 2010 \\
\hline & $\begin{array}{l}\text { Country Economic } \\
\text { Memorandum }\end{array}$ & October 2010 & December 2010 \\
\hline \multirow{2}{*}{$\begin{array}{l}\text { IMF work program in the next } \\
12 \text { months }\end{array}$} & Second review of the ECF & September 2010 & December 2010 \\
\hline & Third review of the ECF & February 2010 & May 2010 \\
\hline \multicolumn{4}{|c|}{ B. Requests for work program inputs } \\
\hline Fund request to Bank & $\begin{array}{l}\text { Provide comments on } \\
\text { implications of sector-specific } \\
\text { issues, including developments } \\
\text { in the mining, energy, telecom } \\
\text { and agricultural sectors. }\end{array}$ & None & FY 11 \\
\hline Bank request to Fund & $\begin{array}{l}\text { Macroeconomic scenarios for } \\
\text { DPO and CEM }\end{array}$ & None & FY 11 \\
\hline \multicolumn{4}{|c|}{ C. Agreement on joint products and missions } \\
\hline \multirow{3}{*}{$\begin{array}{l}\text { Joint products in the next } \\
12 \text { months }\end{array}$} & JSAN of new PRSP & February 2011 & June 2011 \\
\hline & $\begin{array}{l}\text { Public Finance Management } \\
(\mathrm{PFM}) \text { technical assistance }\end{array}$ & FY11-12 & FY11-12 \\
\hline & $\begin{array}{l}\text { Banking sector supervision } \\
\text { technical assistance }\end{array}$ & FY11-12 & FY11-12 \\
\hline
\end{tabular}




\section{Democratic Republic of the Congo: Statistical Issues Appendix (As of May 31, 2010)}

\section{Assessment of Data Adequacy for Surveillance}

General: Data provision to the Fund is broadly adequate for surveillance and program monitoring purposes, but upgrades to the national accounts, balance of payments, and government finance statistics would be helpful. In spite of difficulties, the authorities have continued to produce an array of economic and financial statistics, most of which are published in the annual report of the Central Bank of the Congo (BCC) or its monthly statistical bulletin.

National accounts: Annual national accounts are compiled in constant and current prices by the Directorate of Research of the BCC and published yearly. The compilation methodology conforms to the System of National Accounts 1968 (1968 SNA) and is based on the balance sheets of enterprises and surveys of public and semipublic enterprises and agencies, most of which date from the late 1980s. The activities of the traditional sector (including the informal sector) are estimated with extrapolation techniques using industry-specific data that are outdated. As a result, GDP is likely to be underestimated.

With assistance from AFRITAC, work on the national accounts is underway to update the base year to 2005, incorporate the results of a recent 1-2-3 household survey, as well as implement the methodological recommendations of the 1993 SNA. While outputs of this work remain to be validated and officially published, as expected, the 2005 GDP estimates show a considerable upward revision compared to previously published estimates.

Price statistics: Consumer price indices are calculated for Kinshasa by the BCC, the National Statistics Institute (INS), the Institute of Economic and Social Research (IESR), and the Economics Section of the U.S. Embassy in Kinshasa. The IESR also calculates a monthly consumer price index for the Lubumbashi market. The household surveys on which these calculations are based date back to the late 1980s; they need to be updated to account for changes in household consumption patterns and demographic shifts, while geographical coverage should be expanded.

Government finance statistics: The BCC produces aggregated monthly statistics on a cash basis based on its own accounting for the government cash operations. The treasury produces two sets of monthly statistics based on its own records: one relates to the transactions executed through the BCC, the other set attempts to consolidate operations through commercial bank accounts and off-budget operations. These statistics do not rely on an integrated double-entry public accounting system and do not provide sufficient details about the nature of expenditures owing to problems in the expenditure chain. However, the treasury has started to produce quarterly expenditure data reports by ministry and institution.

The current work on tax administration and expenditure control is expected to improve the quality and timeliness of fiscal statistics. In parallel with technical assistance on public expenditure management, a STA multisector statistics advisor has been advising on government finance statistics, producing improvements in the quality of the statistics. Furthermore, adoption of a simplified double-entry bookkeeping system should significantly improve the availability of data on government financial operations.

In 2003 the DRC reported annual data up to 2002 for the IMF's Government Finance Statistics Yearbook in the format of the Government Finance Statistics Manual 2001, but has not reported subsequently.

Monetary statistics: The BCC Directorate of Research regularly produces timely monetary statistics. These statistics are now generally reliable, but some problems remain with the classification of accounts.

Since STA money and banking statistics mission of June 2000, BCC reporting of data for publication in the IMF's International Financial Statistics (IFS) has improved and monetary data are currently reported on time. A 2003 multisector statistics mission recommended a new chart of accounts for the BCC and other deposit-taking institutions, including the information needed for improved sectorization of economic units and classification of financial assets.

Balance of payments: Annual balance of payments statistics are compiled with information on the exports and imports of large public and semipublic enterprises, BCC payment records, and a survey of the foreign operations of residents. The estimates also take account of information on the informal sector and foreign aid flows provided by the World Bank and the local UN Development Program (UNDP) office, which collects the data from the European Union, embassies, and nongovernmental organizations. However, the 2003 multisector mission found that, due to computer problems, data have not been processed since 1999, so in effect balance of payments statistics are compiled using data from different sources, supplemented by estimates. The mission suggested that the BCC implement a system of quarterly surveys of corporations that are authorized to hold accounts overseas. The mission also recommended reinvigoration of a working group comprising staff of the customs and other agencies to prepare quarterly foreign trade data and take measures to improve data on services and transfers. No external sector data are reported to STA for publication.

\section{Data Standards and Quality}

Since April 24, 2004, the Democratic Republic of the Congo participates in the general Data Dissemination System (GDDS). The metadata needs to be updated. 


\begin{tabular}{|c|c|c|c|c|c|}
\hline \multicolumn{6}{|c|}{$\begin{array}{l}\text { The Democratic Republic of the Congo: Table of Common Indicators Required for Surveillance } \\
\text { (As of May 31, 2010) }\end{array}$} \\
\hline & $\begin{array}{c}\text { Date of latest } \\
\text { observation }\end{array}$ & $\begin{array}{l}\text { Date } \\
\text { received }\end{array}$ & $\begin{array}{c}\text { Frequency of } \\
\text { Data }^{6}\end{array}$ & $\begin{array}{l}\text { Frequency of } \\
\text { Reporting }\end{array}$ & $\begin{array}{l}\text { Frequency of } \\
\text { Publication }{ }^{6}\end{array}$ \\
\hline Exchange Rates & 28-May-10 & 1-Jun-10 & $\mathrm{D}$ & W & $\mathrm{D}$ \\
\hline International Reserve Assets and Reserves & Apr-10 & May-10 & M & M & M \\
\hline Liabilities of the Monetary Authorities ${ }^{1}$ & Apr-10 & May-10 & M & M & M \\
\hline Reserve/Base money & Apr-10 & May-10 & M & M & M \\
\hline Broad money & Apr-10 & May-10 & M & M & M \\
\hline Central Bank Balance Sheet & Apr-10 & May-10 & M & M & M \\
\hline Consolidated Balance Sheet of the Banking System & Apr-10 & May-10 & M & M & M \\
\hline Interest Rates ${ }^{2}$ & Apr-10 & May-10 & M & M & M \\
\hline Consumer Price Index & 28-May-10 & 1-Jun-10 & W & W & W \\
\hline Central Government revenue, expenditure, and financing ${ }^{3}$ & Apr-10 & May-10 & M & M & M \\
\hline Stock of Central Government and & & & & & \\
\hline Central Government-Guaranteed Debt ${ }^{4}$ & Apr-10 & May-10 & A & A & I \\
\hline External Current Account Balance & Apr-10 & May-10 & A & A & I \\
\hline Exports and Imports of Goods and Services & Apr-10 & May-10 & A & A & I \\
\hline GDP/GNP & Apr-10 & May-10 & A & A & I \\
\hline Gross External Debt & Apr-10 & May-10 & A & A & I \\
\hline International Investment Position ${ }^{5}$ & $\mathrm{NA}$ & $N A$ & NA & NA & NA \\
\hline
\end{tabular}

${ }^{1}$ Any reserves assets that are pledged or otherwise encumbered should be specified separately. Also, data should comprise short-term liabilities linked to a foreign currency but settled by other means as well as the national values of financial derivatives to pay and receive foreign currency, including those linked to a foreign curreny but settled by other means.

${ }^{2}$ Both market-based and officially-determined, including discount rates, money markets rates, rates on treasury bills, notes and bonds.

${ }^{3}$ Foreign, domestic bank, and domestic nonbank financing.

${ }^{4}$ Including currency and maturity composition.

${ }^{5}$ Includes external gross financial asset and libility positions vis-à-vis nonresidents.

${ }^{6}$ Daily(D), Weekly (W), Monthly (M), Quarterly (Q), Irregular (I), Not available (NA). 
June 30, 2010

\section{IMF Executive Board Completes First Review Under ECF Arrangement for the Democratic Republic of the Congo and Approves US\$73.20 Million Disbursement}

The Executive Board of the International Monetary Fund (IMF) today completed its first review of the Democratic Republic of Congo's economic performance under the Extended Credit Facility (ECF) arrangement. Completion of the review enables the Democratic Republic of Congo to draw SDR 49.493 million (about US\$73.20 million) immediately, bringing total disbursements under the arrangement to SDR 98.986 million (US\$146.39 million).

The Executive Board approved the three-year ECF arrangement for the Democratic Republic of the Congo (DRC) in December 2009 (see Press Release No. 09/455) in the amount of SDR 346.45 million (about US\$512.36 million, or 65 percent of the country's quota in the Fund).

The Executive Board also agreed, in principle, that the DRC has taken the steps necessary to reach its completion point under the Enhanced Heavily Indebted Poor Countries (HIPC) Initiative. This decision on the HIPC completion point is contingent upon the Executive Board of the World Bank reaching a similar decision, after which a joint press release will be issued.

Following the Executive Board's discussion of the Democratic Republic of the Congo, Mr. John Lipsky, First Deputy Managing Director and Acting Chair, issued the following statement:

"The Democratic Republic of the Congo authorities implemented satisfactorily their economic program despite difficult economic, social, and security conditions. The DRC has met the conditions for reaching the completion point under the Enhanced Heavily Indebted 
Poor Countries Initiative and qualifies for additional debt relief under the Multilateral Debt Relief Initiative (MDRI). Debt relief will significantly reduce the country's debt burden and create fiscal space for poverty reduction programs.

"Going forward, prudent and credible fiscal policy will be key to entrenching macroeconomic stability and making progress toward fiscal sustainability. The authorities' 2010 fiscal policy aims at further reducing government recourse to central bank financing. Effective implementation of revenue-enhancing measures and strict adherence to the expenditure commitment and treasury plans will be critical to achieving the fiscal objectives.

"Safeguarding debt sustainability after HIPC/MDRI debt relief requires prudent debt management. The authorities are committed to meeting their external financing needs through grants and highly concessional loans.

"Monetary policy will continue to focus on containing inflation. The policy interest rate will be kept significantly positive in real terms in order to avoid the reemergence of pressures on the exchange rate and inflation. Recent steps to enhance coordination between the central bank and the Treasury regarding liquidity management should contribute to effective control over the monetary aggregates.

"The authorities intend to accelerate structural reforms aimed at strengthening economic management and bolstering the economy's supply response. These include enhancing revenue administration and public financial management, and addressing weaknesses in the financial sector. The authorities are strongly committed to improving governance and transparency, and to enforcing the rule of law in economic activities, particularly in the mining and oil sectors, critical for foreign direct investment and donor assistance in support of their development objectives." 


\section{Statement by Mr. Rutayisire, Executive Director \\ Democratic Republic of the Congo \\ June 30, 2010}

On behalf of my Congolese authorities, I would like to reiterate our appreciation of the Executive Board's support to the Democratic Republic of the Congo (DRC) in 2009 in the wake of the global financial and economic crisis. I also thank Management and staff for maintaining a constructive and positive dialogue with the DRC authorities throughout challenging years since the decision point under the Enhanced HIPC Initiative in 2003. My authorities agree with the thrust of the staff reports and consented to their publication.

The Democratic Republic of the Congo has come a long way from a period of civil conflicts and macroeconomic instability. Since 2001, the economy of DRC has turned around for the good, although many challenges remain. The early commitment of H.E. Joseph Kabila Kabange to national reconciliation and peace, macroeconomic stabilization, economic reforms and reconstruction has been instrumental to the prompt involvement of the international community, notably the Fund, and the subsequent improvement of the macroeconomic situation. As a result, the DRC has succeeded in maintaining growth rates above 6 percent since 2004, with the noticeable exception of last year when growth decelerated to about 2.8 percent owing to the impact of the global crisis on the domestic economy. The overall implementation of the 2002-06 PRGF-supported program and subsequent staff-monitored programs (SMPs), while not always smoothly implemented due to capacity constraints, repeated conflicts, calamities and external shocks, testify of the authorities' willingness and ability to pursue sound macroeconomic policies and reforms.

With the aim at furthering macroeconomic stability and reaching the HIPC completion point to help bring the distressed debt profile back to a sustainable situation, the DRC has embarked on a medium-term economic program supported by the Fund's ECF. This program notably aims at fostering sustained high growth, enhancing external sustainability and strengthening institutional capacity for improved economic management.

My DRC authorities have met all triggers required to achieve the HIPC completion point. Notably, they have satisfactorily prepared and implemented during the 12 months leading to this date a poverty-reduction strategy laid out in their full PRSP which, according to the recent JSAN, resulted in high budget execution rates and budgetary allocations for priority spending and in good initial results on social outcomes. Second, they have maintained macroeconomic stability as shown by good performance under their current IMF-supported program. Third, my DRC authorities have adequately allocated budgetary savings from interim debt service relief to key areas with greatest impact on poverty, notably security and social sectors. Fourth, the authorities have implemented the three structural measures set at the decision point to strengthen public financial management, namely the implementation of a modernized budget-execution system, the adoption and implementation of a double-entry government accounting system and a new chart of accounts, and the production of quarterly budget execution reports using economic, administrative and functional classifications. Fifth, regarding governance and service delivery in priority sectors, the authorities have 
successfully completed a budget-tracking exercise to monitor the execution of social sector expenditures. They have also adopted in April 2010 - and are implementing - a new procurement code and key implementing decrees. Sixth, on the trigger related to social and rural sectors, the government adopted this year sectoral development strategies and related action plans for health, education and rural development on the basis of reliable analyses and consultations with stakeholders. Finally, the DRC authorities have met the trigger on debt management enhancement by putting in place and activating a computerized debt-recording system covering all the various types of public and publicly-guaranteed debt. As a result, monthly debt service statements are produced on a quarterly basis. All debt information is now centralized at the Direction Générale de la Dette Publique (DGDP, formerly OGEDEP).

To address the capacity constraints and other perceived risks to the program, my DRC authorities have, in agreement with Fund staff, designed a series of reforms, notably to enhance public financial management and strengthen the central bank through restructuring and recapitalization. They will continue to reinforce the government's monitoring mechanisms they have put in place both at the political and technical levels, through interministerial consultations and exchange of information, under the coordination and supervision of the Prime Minister's Office.

Based on the performance achieved thus far under the medium-term program as indicated by the observance of all quantitative performance criteria at end-December 2009 and the implementation of almost all of the structural benchmarks - albeit with delays due to capacity weaknesses - and in light of renewed commitment to their economic program looking forward, my DRC authorities request the completion of the first review under the ECF. They also request the completion of the financing assurances review, having secured the full financing of the program, with the remaining gap expected to be covered by external budget support from the World Bank, the EU and bilateral donors, and exerting efforts toward reaching agreements with commercial creditors. In addition, the authorities request the Executive Board support to DRC's completion point under the Enhanced HIPC Initiative on the basis of the country having met all the related triggers.

\section{RECENT ECONOMIC DEVELOPMENTS AND PROGRAM IMPLEMENTATION}

The DRC's macroeconomic performance has been satisfactory, with all quantitative PCs at end-December 2009 observed. The authorities have pursued macroeconomic policies aimed at mitigating the effects of the global recession while maintaining the program quantitative objectives. On the fiscal policy front, the domestic fiscal deficit widened in 2009 to allow an increase in investment in infrastructure. In the first quarter of 2010, there have been signs of improvements in the economy following the adverse impact of the global crisis on the commodity exports price, mining activity and FDI. As a result, government revenue strengthened and more than offset the modest spending overruns due to national security, preparation of the $50^{\text {th }}$ independence anniversary celebrations and cost of monetary policy operations.

Regarding monetary policy, there have been improvements in monetary control since the adoption of the Fund-supported program, owing to continued good fiscal performance and 
BCC's efforts to enhance its liquidity forecasting, in collaboration with the Treasury. As inflation has declined, the BCC eased monetary policy stance by gradually cutting its policy interest rate to 42 percent by May 2010 from 70 percent at the time of the program approval in December 2009.

On the structural front, significant progress has also been registered. Most benchmarks through March 2010 met albeit with delays due to capacity constraints. Concerning the delays on structural measures, the restructuring of a systemically-important commercial bank has required more time than initially envisaged, and the central bank (BCC) launched the restructuring process in May 2010 in consultation with Fund's MCM. Second, regarding the benchmark on an organic budget law, the Government submitted to parliament a budget finance law that is consistent with the Constitution after an initial draft "organic" law was deemed inconsistent with the Constitution. Finally, difficulties in obtaining information on several foreign financed projects prevented the observance of a continuous benchmark on timely production of fiscal accounts with foreign financing spending.

Good performance is being registered in the areas of (i) public financial management, with the adoption of an action plan to strengthen PFM; (ii) governance and transparency in the mining and oil sectors, with preparation underway to adhere to the EITI; and (iii) business environment, with the promulgation of the protocol of the OHADA. The latter measure will help reduce the cost of doing business.

More broadly, as improving the business climate is at the core of their actions to promote private sector investment, my authorities have put in place a ministerial committee (Comité de Pilotage pour l'Amélioration du Climat des Affaires) to make significant progress on this front. While more remains to be done, sixteen (16) important measures have been taken to date, in accordance with two roadmaps adopted by the ministerial council a few months ago. Measures already taken include the streamlining of documents required for business registration, the reduction and publication of business registration fees, the reduction in the time required to obtain the business identification number, the decentralization of the notary function, the cancellation of the legal visa for the status of associates, and the publication of businesses' acts.

\section{Policies AND REFORMS FOR THE REMAINDER OF 2010}

In pursuing efforts to boost the economy, my DRC authorities remain determined to enhance public financial management, strengthen fiscal governance, maintain macroeconomic stability and their program's macroeconomic objectives, pursue prudent debt policy based on grants and highly concessional borrowing, and advance structural reforms in key growthenhancing areas, notably the mining, oil, and financial sector.

\section{A. Fiscal Policy}

The authorities remain committed to a fiscal policy that pursues less financing from the central bank. They reconfirmed their commitment to implement such prudent fiscal policy. The program for the remainder of 2010 envisages revenue-enhancing measures and reprioritization of spending to create the necessary fiscal space for infrastructure and other 
priority spending, notably in education, health, agriculture and rural development. The revenue measures include a gradual alignment of the price used for the calculation of taxes on petroleum products, enforcement of the payment of income taxes by political institutions, and the elimination of ad hoc tax and customs exemptions.

On the expenditures side, the new expenditure management procedures recently introduced, and which also apply to urgent spending, should help strengthen expenditure control. The authorities have committed to saving a portion of the excess domestic revenue and the full amount of the second tranche of the SCCA signing bonus and will consult Fund staff for any use of these resources.

\section{B. Monetary and Exchange Rate Policies}

The goal of monetary policy remains price stability under the floating exchange rate regime, which has served the economy well as a buffer to external shocks. To make monetary policy more effective, the BCC will continue to strengthen its policy framework by introducing more flexibility in the indirect monetary policy instruments (open market operations and reserve requirements) and further enhancing its liquidity management and forecasting capacity, in greater collaboration with the Treasury. The central bank will intervene in foreign exchange market only to smooth out large exchange rate fluctuations.

\section{Financial Sector Policies}

The authorities will strive to enhance the BCC's operational capacity and put it on a sound financial footing through restructuring and recapitalization, with technical assistance from the IMF. They have adopted an action plan to improve governance and strengthen the central bank's operational effectiveness. The recapitalization of the Bank, to be provided by the government, is expected in 2011.

Regarding the banking sector, the authorities aim at safeguarding financial system stability and soundness through an improved regulatory framework, including increased minimum capital requirements for banks and more rigorous licensing procedures; enhanced supervision notably in human capacity; strengthened on- and off-site inspection; and macro-prudential functions. The central bank will complete ongoing efforts to address problems faced by one large bank with systemic importance.

\section{Other Structural Reforms}

Structural reforms for the remainder of 2010 will build on the progress achieved thus far to further revenue mobilization, focus on PFM strengthening, and promote private sector development. Regarding revenue mobilization, tax and customs administration will be strengthened through the adoption of a VAT law, the adoption of new tax centers in key provinces for medium-sized enterprises, and expansion off one-stop windows at the borders.

As regards the public financial management, the adoption of the new finance law will streamline budget planning, execution and monitoring. It will also lay the grounds for a 
phased devolution of social expenditures from the central government to the provinces, taking into account the PFM capacities at the provincial level.

Concerning efforts to promote private sector development, planned reforms include notably advancing the ongoing restructuring of public enterprises, streamlining regulations and harmonizing it with the OHADA protocol, and adhering to the EITI. In addition, the authorities will pursue the adoption of measures envisaged in the roadmaps to improve the business climate and work on the related application decrees.

\section{Debt Sustainability Analysis and HiPC Completion Point}

The updated debt sustainability analysis conducted jointly by Fund and World Bank staffs show that the DRC remains in debt distress. Absent debt relief under the HIPC Initiative and MDRI, its external debt burden indicators are projected to remain above the policydependent-thresholds over a protracted period even with a strong recovery over the medium term. As debt distress continues to exert a drag on the economy, the DRC is in dire need to reach the HIPC completion point. As envisaged at the approval of the ECF-supported program, the DRC authorities have stepped up efforts to meet all remaining completion point triggers. These include the continued maintenance of macroeconomic stability; the use of budgetary savings from HIPC Initiative-related debt service relief for poverty-related expenditures; and measures in the areas of public financial management, governance and service delivery in priority sectors, social and rural sector strategies, and strengthening of debt management. The trigger on the PRSP process had been met before since the implementation of the full participatory PRSP adopted in 2006 was satisfactory, according to the most recent JSAN. The authorities are now working on a second-generation PRSP.

Reaching the completion point will provide substantial debt relief and allocate freed domestic resources and additional external support to the DRC's poverty reduction and reconstruction efforts.

Furthermore, even with the HIPC/MDRI debt relief, the country's external outlook would remain vulnerable to adverse shocks. Therefore, the authorities fully understand the need, on their part, to maintain a prudent debt management policy. After amending an important cooperation agreement aimed at building and modernizing infrastructure, while revitalizing the mining sector, to make it consistent with program-related concessionality requirements and with debt sustainability, they recently reiterated their commitment to refrain from contracting or guaranteeing new debt that would jeopardize debt sustainability. Moreover, my DRC authorities have secured assistance from the Paris Club creditors, multilateral creditors and many commercial creditors. They also maintain dialogue with London Club and non-London Club private creditors, with the view to obtain debt restructuring on comparable terms.

\section{CONCLUSION}

As a post-conflict country facing high debt distress, the Democratic Republic of the Congo is at a crossroads of its economic and development future. In this regard, reaching the completion point is a critical milestone. Full delivery of debt relief from all sources at the 
completion point would reduce its external public debt burden considerably and the external debt outlook is expected to improve markedly over the long term. In this vein, my DRC authorities have met all completion point triggers under the Enhanced HIPC Initiative. They have obtained assurances from all Paris Club creditors, nearly all multilateral creditors, some other official creditors, and several commercial creditors for participation in the assistance to the DRC under the Enhanced HIPC Initiative, and are working toward securing the participation of the remaining creditors.

At the same time, as the updated DSA indicates, there is a need for strong and continued efforts to diversify the economy, reduce the risk of adverse shocks and ensure prudent debt management in order to maintain low debt vulnerabilities. My authorities strive to address these challenges within their medium-term Fund-supported program as stressed in their recent letter of Intent and MEFP. They stand ready to take any further measures that may prove necessary for achieving their program's objectives.

On the basis of all the performances achieved, my DRC authorities would greatly appreciate the Executive Board's support of the completion of the First Review under the ECF, the completion of the financing assurances review and the completion point under the Enhanced HIPC Initiative and MDRI. 